SUBMITTED

Preprint typeset using $\mathrm{LAT}_{\mathrm{E}} \mathrm{X}$ style emulateapj v. 12/16/11

\title{
PHOTOMETRIC OBSERVATIONS OF SUPERNOVA 2013CQ ASSOCIATED WITH GRB 130427A
}

\author{
Becerra, R. L. ${ }^{1}$, Watson, A. M. ${ }^{1}$, Lee, W. H. ${ }^{1}$; Fraija, N. ${ }^{1}$; Butler, N. R. ${ }^{2}$; Bloom, J. S. ${ }^{3}$; Capone, J. I. ${ }^{4}$; \\ Cucchiara, A. ${ }^{5}$; de Diego, J. A. ${ }^{1}$; Fox, O. D. ${ }^{6}$ Gehrels, N. ${ }^{7}$; Georgiev, L. N. ${ }^{1}$; González, J. J. ${ }^{1}$; Kutyrev, A. S. ${ }^{7}$; \\ Littlejohns, O. M. ${ }^{2}$; Prochaska, J. X. ${ }^{8}$; Ramirez-Ruiz, E. ${ }^{8}$; Richer, M. G. ${ }^{9}$; Román-ZúNiga, C. G. ${ }^{9}$; Toy, V. L. ${ }^{4}$; \\ TrojA, E. ${ }^{4,7}$ \\ ${ }^{1}$ Instituto de Astronomía, Universidad Nacional Autónoma de México, Apartado Postal 70-264, 04510 México, D. F., México; \\ 2 School of Earth and Space Exploration, Arizona State University, Tempe, AZ 85287, USA; \\ ${ }^{3}$ Department of Astronomy, University of California, Berkeley, CA 94720-3411, USA; \\ ${ }^{4}$ Department of Astronomy, University of Maryland, College Park, MD 20742, USA; \\ ${ }^{5}$ NASA Postdoctoral Program Fellow, Goddard Space Flight Center, Greenbelt, MD 20771, USA; \\ ${ }^{6}$ Space Telescope Science Institute, 3700 San Martin Drive, Baltimore, MD 21218, USA; \\ ${ }^{7}$ NASA, Goddard Space Flight Center, Greenbelt, MD 20771, USA; \\ ${ }^{8}$ Department of Astronomy and Astrophysics, UCO/Lick Observatory, University of California, 1156 High Street, Santa Cruz, CA 95064, \\ USA; \\ ${ }^{9}$ Instituto de Astronomía, Universidad Nacional Autónoma de México, Apartado Postal 106, 22800 Ensenada, Baja California, México;
}

Draft version November 13, 2018

\begin{abstract}
We observed the afterglow of GRB 130427A with the RATIR instrument on the 1.5-m Harold L. Johnson telescope of the Observatorio Astronómico Nacional on Sierra San Pedro Mártir. Our homogenous griZYJH photometry extends from the night of burst to three years later. We fit a model for the afterglow. There is a significant positive residual which matches the behavior of SN $1998 \mathrm{bw}$ in the griZ filters; we suggest that this is a photometric signature of the supernova SN $2013 \mathrm{cq}$ associated with the GRB. The peak absolute magnitude of the supernova is $M_{r}=-18.43 \pm 0.11$.
\end{abstract}

\begin{abstract}
Subject headings: (stars) gamma-ray burst: individual (GRB 130427A) - (stars:) supernovae: individual (SN 2013cq).
\end{abstract}

\section{INTRODUCTION}

Gamma-ray bursts (GRBs) are the most energetic events in the universe and are produced at cosmological distances. They can be classified according to their duration $T_{90}$, the time interval in the observer's frame over which $90 \%$ of the total background-subtracted counts are observed (Koshut et al. 1995). This parameter has long pointed to a bi-modal distribution (Kouveliotou et al. 1993).

Long GRBs $\left(T_{90}>2 \mathrm{~s}\right)$ are today thought to be the result of the core-collapse of a star (Woosley 1993; MacFadyen \& Woosley 1999; Hjorth \& Bloom 2012) with an initial mass with more than $10 \mathrm{M}_{\odot}$ (see Woosley \& Bloom 2006, for a review), while short GRBs $\left(T_{90}<2\right.$ s) are thought to be the result of mergers between two compact objects (Lattimer \& Schramm 1976; Paczynski 1986, 1991; Eichler et al. 1989; Narayan, Paczynksi \& Piran 1992) like black holes or neutron stars (see Lee \& Ramirez-Ruiz 2007; Nakar 2007, for reviews).

Woosley (1993) specifically proposed a way in which the core collapse of massive stars could lead to a long GRB, and thus be possibly associated with a supernova (SN) (Modjaz 2011; Woosley \& Bloom 2006; Bersier 2012; Hjorth 2013). In this scenario, the optical emission from the SN would appear a few days after the GRB, when the ejecta becomes optically thin. This leads to two ways to identify the presence of a SN associated with a GRB. First, by the appearance of the broad spectral lines characteristic of SN a few days after the burst, and second, through a rebrightening in the lightcurve of the GRB after a few days due to the broad-band emission of the SN. The identification of a SN associated with GRB 980425 (Galama et al. 1998) showed that at least some GRBs are truly linked to the core collapse of massive stars.

Subsequently, other SNe have also been associated with long GRBs. Confirmed spectroscopic cases are listed in Table 1. Most or perhaps all of these SNe are type Ic. Usually the hosts of GRB-SNe are blue, star-forming galaxies (Fynbo et al. 2000; Foley et al. 2006; Hammer et al. 2006; Fruchter et al. 2006; Niino 2013) and the events occur within a low metallicity environment (Modjaz et al. 2008).

Simply taking into account the rates of SNe and GRBs, however, it is apparent that not all core-collapse $\mathrm{SNe}$ produce long GRBs, and special conditions are required in order to successfully power a burst. These probably involve rotation, magnetic fields, chemical composition, binarity, or a combination of the above and are not yet fully resolved. Thus, precise observations of a significant sample of GRBs associated with SNe are fundamental in order to determine the evolutionary pathways that can lead to such a link. Given that the current sample is extremely limited, studying single events like SN 2013cq associated with GRB 130427A in great detail offers the opportunity to provide unique additional insights and to eventually lead the way to a statistically significant sample, and this is the main motivation for the present paper. 
GRB 130427A is one of the most brightest gammaray burst in the last few years, and had $E_{\gamma, \text { iso }}=1.40 \times$ $10^{54} \mathrm{erg}$ in total isotropic energy release (Ackermann et al. 2014) and $E_{\gamma \text {,peak }}=1028 \pm 8 \mathrm{keV}$ (Maselli et al. 2014). It was detected at high energies by several satellite-borne instruments and lead to a flurry of ground-based observations. In total, there have been 91 GCN Circulars related to GRB $130427 \mathrm{~A}$. Its redshift was measured to be $z=0.34$ (Levan et al. 2013). RAPTOR (Rapid Telescope for Optical Response) observed a bright optical flash with a magnitude of $7.03 \pm 0.03$ in the time interval from $9.31 \mathrm{~s}$ to $19.31 \mathrm{~s}$ after the GBM trigger (Vestrand et al. 2014). The bright optical flash at early times was modeled with synchrotron emission from reverse shocks (Vestrand et al. 2014; Fraija et al. 2016). Perley et al. (2014) show multi-wavelength optical/infrared photometry of the afterglow of GRB 130427A, and explain the afterglow through synchrotron radiation and suggest a massive-star progenitor. Spectroscopy with the 10.4-m GTC telescope reported by $\mathrm{Xu}$ et al. (2013) showed a broad-lined Ic SN 2013cq associated with GRB 130427A.

This paper presents a detailed set of calibrated and uniform photometry of the bright GRB 130427A with the RATIR instrument in the griZYJH filters. The major advantages of our work compared to earlier papers (Perley et al. 2014; Xu et al. 2013; Melandri et al. 2014) is that our photometry is generally deeper, has better temporal sampling, and we subtract the host galaxy using deep late-epoch image. Furthermore, our data were all obtained with the same instrument, using the same observing strategy, and were all processed in the same way. This means that our data are naturally homogeneous. The paper is organized as follows: in $\S 2$ we present the observations; in $\S 3$ we fit the data using segments of power-law according to the fireball model;

in $\$ 4$ search for the signature of $S N 2013 \mathrm{cq}$ in the the difference between the host-subtracted measurements and the power-law afterglow model;

in $\S 5$ we discuss the results and summarize our conclusions.

\section{OBSERVATIONS}

\subsection{Fermi and Swift}

The Gamma-Ray Burst Monitor (GBM) instrument on the Fermi satellite triggered on GRB 130427A at 07:47:06.42 UTC on 2013 April 27 (von Kienlin 2013). Subsequently, the Burst Alert Telescope (BAT) on the Swift satellite triggered on the GRB at 07:47:57.51 UTC (Maselli et al. 2014). The duration measured with BAT was $T_{90}=163 \mathrm{~s}$ (Barthelmy et al. 2013), making GRB 130427A a long GRB.

\subsection{RATIR}

The Reionization and Transients Infrared Camera (RATIR) is a four-channel simultaneous optical and near infrared imager mounted on 1.5-m Harold L. Johnson Telescope at the Observatorio Astronómico Nacional on Sierra San Pedro Mártir in Baja California, Mexico. RATIR responds autonomously to GRB triggers from the Swift satellite and obtains simultaneous photometry in riZJ or riYH (Butler et al. 2012; Watson et al. 2012; Littlejohns et al. 2015). In manually-programmed follow-up observations, the $g$ filter can be substituted for $r$.

RATIR began to observe the field of GRB 130427A 15.5 minutes after the BAT trigger, and continued to observe it intensively over the subsequent weeks. On the first night, the $r$ detector failed, so we only have data in $i Z Y J H$. On subsequent nights, we have data in riZYJH. After one week, we began to observe in $g$ as well. We reobserved the field on several nights in 2014 and 2016 mainly to place constraints on the host galaxy.

Our reduction pipeline performs bias subtraction and flat-field correction, followed by astrometric calibration using the astrometry.net software (Lang et al. 2010), iterative sky-subtraction, coaddition using SWARP, and source detection using SEXTRACTOR (Littlejohns et al. 2015). We calibrate against SDSS and 2MASS (Littlejohns et al. 2015). The systematic calibration error is about $1 \%$.

The individual exposures were $80 \mathrm{~s}$ in gri and $67 \mathrm{~s}$ in $Z Y J H$ filters (with the infrared exposures being shorter because of their longer read-out overhead). On the first night, we consider the exposures individually. For the second to the fifth night, we combined sets of 16 exposures taken over about 30 minutes to improve the signalto-noise ratio. For the remaining nights in 2013, we combined all of the exposures for each night, for 2014, we combined several nights, and for 2016, we combined all of the exposures. The image quality in the final images was typically 2 arcsec FWHM.

We obtained aperture photometry using a 3 arcsec diameter aperture. Table 6 gives our aperture photometry. For each image it gives the start and end time, $t_{0}$ and $t_{f}$, the total exposure time $t_{e}$, the magnitude, the $1 \sigma$ total uncertainty (including both statistical and systematic contributions), and the filter. These magnitudes are not corrected for Galactic extinction.

We also obtained PSF-fitting photometry of the afterglow and supernova in our 2013 and 2014 images after subtracting the host galaxy using our 2016 image. For each image, we estimated the point-spread function (PSF) by co-aligning and summing images of stars (as categorized by the SDSS) within 3 arcmin of the GRB. We subtracted the 2016 image from the earlier images using HOTPANTS (Becker 2015) and fitted the PSF to the residual. Even though our image quality is typically 2 arcsec FWHM, we cannot reliably perform PSF-fitting on the unsubtracted images because the galaxy is offset about 0.8 arcsec to the south-east of the afterglow (Levan et al. 2014). Table 6 also gives our PSF-fitting photometry. The main advantage of PSF-fitting is that the statistical uncertainties are reduced typically by about $20 \%$. Figure 1 shows the RATIR optical and near-infrared light curves.

\section{MODELS}

The standard fireball model for GRBs (Kumar \& Zhang 2015) distinguishes two stages: the prompt emission and the afterglow. The prompt emission is simultaneous with emission in gamma rays and is produced by internal shocks in the jet driven by the central engine. The afterglow is produced by the external shock between the jet and the circumstellar environment (e.g. Kumar \& Piran 2000; Fraija 2015).

The emission region of the radiation determines the form and behavior of the spectrum and light curve for 


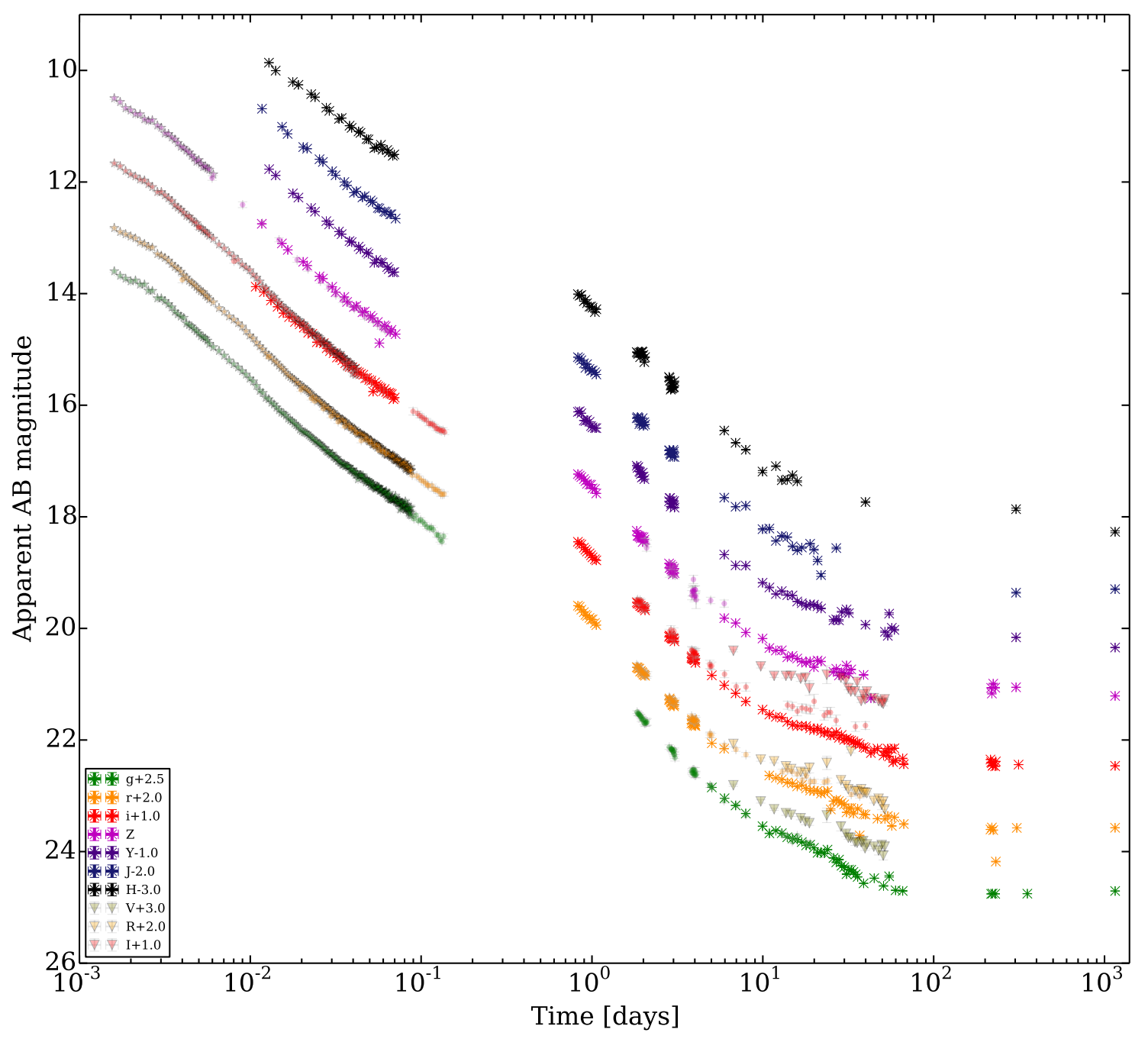

FIG. 1. - griZYJH light curves for GRB 130427A from RATIR (lines), RAPTOR (Maselli et al. 2014) (stars) and Palomar P60 (points) (Perley et al. 2014) and VLT (Melandri et al. 2014) (triangles). The RATIR photometry shown here is our aperture photometry of the afterglow and host galaxy.

a GRB (e.g. Fraija et al. 2016), and can be different for each filter. Optical radiation has three possible origins: internal shocks in the jet, the forward external shock, and the reverse external shock (Sari \& Piran 1999).

The afterglow phase can be explained by assuming a power-law energy distribution of shocked relativistic electrons, $N(E) \propto E^{-p}$, which lead to the observed flux being a series of power-laws segments as a function of time $t$ and frequency $\nu$ as $F_{\nu}^{\mathrm{syn}} \propto t^{-\alpha} \nu^{-\beta}$ (Sari et al. 1998).

RATIR began to observe after the end of the prompt emission, so we only have photometry for the afterglow. We divided these data into two epochs: the early after- glow for time $t<0.1$ days (the first night) and the late afterglow for the $t>0.7$ day (the second and subsequent nights). This division was guided by the analysis of Perley et al. (2014), who report a change in the slope of the light curve at $t=0.7$ days. We have no data between $t=0.1$ and $t=0.7$ days.

\subsection{Host galaxy}

Volnova et al. (2013) suggested that SDSS DR12 galaxy object 1237667431180861948 was the host galaxy of the GRB. This was subsequently confirmed by the close agreement in redshift between absorption lines in 
TABLE 1

GRBS WITH ASSOCIATED SN

\begin{tabular}{clcccc}
\hline \hline \multicolumn{1}{c}{ GRB } & \multicolumn{1}{c}{ SN } & SN type & z & Evidence $^{\mathrm{a}}$ & \multicolumn{2}{c}{ References $^{\text {SN }}$} \\
\hline GRB 980425 & SN 1998bw & Ic & 0.0085 & A & 1 \\
GRB 011121 & SN 2001ke & IIn?/Ic? & 0.362 & B & $2,3,4$ \\
GRB 021211 & SN 2002lt & Ic & 1.006 & B & 5 \\
GRB 030329 & SN 2003dh & Ic & 0.1687 & A & 6,7 \\
GRB 031203 & SN 2003lw & Ic & 0.105 & A & $8,9,10$ \\
GRB 050525A & SN 2005nc & Ic & 0.606 & B & 11 \\
GRB 060218 & SN 2006aj & Ic & 0.0335 & A & $12,13,14,15,16$ \\
GRB 081007 & SN 2008hw & Ic & 0.530 & B & $17,18,19$ \\
GRB 091127 & SN 2009nz & Ic & 0.49 & A & 20,21 \\
GRB 100316D & SN 2010bh & Ic & 0.059 & A & $22,23,24,25,26$ \\
GRB 120422A & SN 2012bz & Ic & 0.283 & A & 27,28 \\
GRB 130427A & SN 2013cq & Ic & 0.34 & A & $29,30,31$ \\
GRB 130702A & SN 2013dx & Ic & 0.145 & A & 32,33 \\
GRB 140606B & iPTF14bfu & Ic & 0.384 & A & 34 \\
\hline
\end{tabular}

References. - (1) Galama et al. (1998); (2) Bloom et al. (2002); (3) Garnavich et al. (2003); (4) Greiner et al. (2003); (5) Della Valle et al. (2003); (6) Stanek et al. (2003); (7) Matheson et al. (2003); (8) Malesani et al. (2004); (9)Gal-Yam et al. (2004); (10) Thomsen et al. (2004); (11) Della Valle et al. (2006); (12) Campana et al. (2006); (13) Modjaz et al. (2008); (14) Mirabal et al. (2006); (15) Ferrero et al. (2006); (16) Pian et al. (2006); (17) Berger et al. (2008); (18) Della Valle et al. (2008);(19) Soderberg et al. (2008); (20) Cobb et al. (2010); (21) Berger et al. (2011); (22) Starling et al. (2011); (23) Bufano et al. (2012); (24) Olivares E. et al. (2012); (25) Cano et al. (2011); (26) Chornock et al. (2010); (27) Melandri et al. (2012); (28) Schulze et al. (2014); (29) de Ugarte Postigo et al. (2013); (30) Xu et al. (2013); (31) This work; (32) Toy et al. (2015); (33) D'Elia et al. (2015); and (34) Cano et al. (2015).

a Evidence for the GRB-SN association, according to the authors, with A meaning strong spectroscopic evidence and B meaning a clear light curve bump together with some spectroscopic evidence resembling a $\mathrm{SN}$.

b SN 2001 ke has no clear spectroscopic classification. Garnavich et al. (2003) suggest that it is a type IIn but Bloom et al. (2002) claim it is indeed consistent with a 1998bw-like type Ic.

the GRB spectrum (Levan et al. 2013; Xu et al. 2013; Flores et al. 2013) and emission lines from the galaxy (Xu et al. 2013).

Levan et al. (2014) obtained HST images of the afterglow and host galaxy. They suggested that the host is a moderately star-forming, possibly interacting, disk galaxy and the GRB occured about $0.8 \operatorname{arcsec}(4 \mathrm{kpc})$ from the nucleus.

Table 2 reports our RATIR griZYJH aperture magnitudes from 2016 and magnitudes from the SDSS DR12 image using the same apertures and calibrating stars. The magnitudes from our image are consistent with the magnitudes from the SDSS image, but have lower uncertainties.

We can estimate the rest-frame $g-i$ color and $M_{i}$ magnitude from our observed $r-Y$ color and $Y$ magnitude (see Table 4 for the correspondence between rest-frame and observed bands) assuming a $\Lambda$ CDM model with a $H_{0}=67.8 \mathrm{~km} / \mathrm{Mpc} / \mathrm{s}$ (Planck Collaboration et al. 2014). We obtain a rest-frame $g-i=0.23 \pm 0.08$ and a restframe $M_{i}=-19.91 \pm 0.07$. These properties place it among the most extremely blue galaxies in the $z \approx 0$ sample of Gavazzi et al. (2010).

\subsection{Early Afterglow $(t<0.7$ days $)$}

To characterize the early afterglow data $(t<0.7$ days $)$, we used the $i Z Y J H$ aperture photometry from Table 6 as the contribution of the host galaxy can be neglected at early times. We fitted the flux densities with a powerlaw model $F=A_{\mathrm{E}} t^{-\alpha_{\mathrm{E}}}$, in which $F$ is the flux density in the filter, $A_{\mathrm{E}}$ is a constant, $t$ is the time since the BAT trigger (in days), and $\alpha_{\mathrm{E}}$ is the temporal index, assumed to be the same for all filters. The model has six free
TABLE 2

Host-Galaxy MAGNITUDES FOR GRB 130427A

\begin{tabular}{lcrc}
\hline \hline Filter & $m_{\text {RATIR }}$ & Exposure $(\mathrm{h})$ & $m_{\text {SDSS }}$ \\
\hline$g$ & $22.20 \pm 0.04$ & 10.6 & $22.14 \pm 0.12$ \\
$r$ & $21.57 \pm 0.04$ & 6.5 & $21.41 \pm 0.10$ \\
$i$ & $21.46 \pm 0.03$ & 17.8 & $21.53 \pm 0.23$ \\
$Z$ & $21.29 \pm 0.05$ & 8.3 & $\cdots$ \\
$Y$ & $21.34 \pm 0.07$ & 8.2 & $\cdots$ \\
$J$ & $21.30 \pm 0.09$ & 8.0 & $\cdots$ \\
$H$ & $21.27 \pm 0.13$ & 7.9 & \\
& & & \\
\hline
\end{tabular}

parameters: the five values of $A_{\mathrm{E}}$ and the one value of the index $\alpha_{\mathrm{E}}$.

We minimized the value of $\chi^{2}$ to find the best-fit parameters. The final fit has a $\chi^{2} / \mathrm{n}=1.64$ with $n=100$ degrees of freedom. The best-fit parameters are given in Table 3. The errors were calculated using the standard deviation of the best-fits parameters after Gaussian perturbations around the flux values observed over 10,000 trials. Table 7 shows the residuals to the fit in the sense of data minus model in units of $\mu \mathrm{Jy}$.

\subsection{Late Afterglow ( $t>0.7$ days)}

To characterize the late afterglow $(t>0.7$ days $)$, we use the griZYHJ flux densities from our PSF-fitting photometry of the subtracted images from Table 6 . We fitted with a model $F=A_{\mathrm{L}} t^{-\alpha_{\mathrm{L}}}$, in which $F$ is the flux density in each filter, $A_{\mathrm{L}}$ is a constant, $t$ is the time since the BAT trigger (in days) and $\alpha_{\mathrm{L}}$ is the temporal index, assumed to be the same for all filters. The model has eight free parameters: the seven values of $A_{\mathrm{L}}$ and the one value of the index $\alpha_{\mathrm{L}}$. 
TABLE 3

Fit PARAMETERS

\begin{tabular}{ccc}
\hline \hline Parameter & Band & Value \\
\hline$\alpha_{\mathrm{E}}$ & & $0.97 \pm 0.04$ \\
$A_{\mathrm{E}}$ & $i$ & $303 \pm 28 \mu \mathrm{Jy}$ \\
$A_{\mathrm{E}}$ & $Z$ & $348 \pm 38 \mu \mathrm{Jy}$ \\
$A_{\mathrm{E}}$ & $Y$ & $373 \pm 38 \mu \mathrm{Jy}$ \\
$A_{\mathrm{E}}$ & $J$ & $364 \pm 37 \mu \mathrm{Jy}$ \\
$A_{\mathrm{E}}$ & $H$ & $373 \pm 27 \mu \mathrm{Jy}$ \\
\hline$\alpha_{\mathrm{L}}$ & & $1.41 \pm 0.04$ \\
$A_{\mathrm{L}}$ & $g$ & $228 \pm 12 \mu \mathrm{Jy}$ \\
$A_{\mathrm{L}}$ & $r$ & $268 \pm 10 \mu \mathrm{Jy}$ \\
$A_{\mathrm{L}}$ & $i$ & $315 \pm 20 \mu \mathrm{Jy}$ \\
$A_{\mathrm{L}}$ & $Z$ & $388 \pm 37 \mu \mathrm{Jy}$ \\
$A_{\mathrm{L}}$ & $Y$ & $436 \pm 18 \mu \mathrm{Jy}$ \\
$A_{\mathrm{L}}$ & $J$ & $426 \pm 11 \mu \mathrm{Jy}$ \\
$A_{\mathrm{L}}$ & $H$ & $489 \pm 116 \mu \mathrm{Jy}$ \\
\hline
\end{tabular}

Again, we minimized the value of $\chi^{2}$ to find the best-fit parameters. To avoid the worst contamination from the SN, we fitted only the data points from 0.7 to 7 days and from 40 days onwards. The final fit has a $\chi^{2} / n=1.05$ with $n=327$ degrees of freedom. The best-fit parameters are given in Table 3. The errors were calculated using the standard deviation of the best-fits parameters with Gaussian perturbations to the flux value and 10,000 trials. Figures 2 to 8 show the data and the best fit. Table 8 shows the residuals to the fit in the sense of data minus model in units of $\mu \mathrm{Jy}$.

\section{RESULTS}

\subsection{SN Component}

The host-subtracted measurements minus the the bestfit afterglow models (Table 8), henceforth residuals, are show in Figures 2 to 8 . These residuals show a rise and fall from about 7 to about 40 days, confirming the suggestion of Watson et al. (2013). We propose that this is the photometric signature of SN 2013cq.

To compare our data to SN $1988 \mathrm{bw}$ at a redshift of $z=$ 0.0085 (Tinney et al. 1998), we need to account for the effects of redshift on the luminosity distance, observed band, and time dilation. For the luminosity distance, we used a $\lambda \mathrm{CDM}$ model with a $H_{0}=67.8 \mathrm{~km} / \mathrm{Mpc} / \mathrm{s}$ (Planck Collaboration et al. 2014). The effect of redshift on the filters is shown in Table 4. In this table, the first and second column give the RATIR filter and its central wavelength $\bar{\lambda}$ at $z=0$, the third column gives the central wavelength $\bar{\lambda}$ in the rest frame of SN 2013cq at $z=0.34$, and the fourth and fifth give the corresponding JohnsonCousins filters and their central wavelengths at $z=0$. Fortuitously, there is a good correspondence between the RATIR gri $Z Y H$ filters at $z=0.34$ and the Johnson $U B V R I J$ filters at $z \approx 0$. The time dilation correction is a factor of $(1+0.34) /(1+0.0085)=1.33$.

Figure 9 compares our photometry of SN 2013cq with that of SN 1998bw shifted to $z=0.34$ (Galama et al. 1998; Clocchiatti et al. 2011; Foley et al. 2006), both bands in rest frame. Qualitatively, the agreement is good, especially in the bluer griz filters although compared with SN 1998bw, SN 2013cq is fainter in $g$-band while is brighter in the riz filters.

The peak times, calculated by adjusting third-degree polynomials to the residuals (between 7 and 40 days), are $17.66,17.33$ and 22.00 days with $\chi^{2} /$ d.o.f. of 0.62 , 0.92 and 0.06 respectively, for the $g, r$, and $i$ bands, and
TABLE 4

CORRESPONDENCE BETWEEN RATIR FILTERS AT $z=0.34$ AND JOHNSON-COUSINS FILTERS $\mathrm{AT} z \approx 0$

\begin{tabular}{lrrcc}
\hline \hline Filter & $\begin{array}{c}\bar{\lambda}[\mathrm{nm}] \\
z=0\end{array}$ & $\begin{array}{c}\bar{\lambda}[\mathrm{nm}] \\
z=0.34\end{array}$ & Filter & $\begin{array}{c}\bar{\lambda}[\mathrm{nm}] \\
z=0\end{array}$ \\
\hline$g$ & 470 & 351 & $U$ & 360 \\
$r$ & 618 & 461 & $B$ & 440 \\
$i$ & 760 & 567 & $V$ & 550 \\
$Z$ & 878 & 655 & $R$ & 640 \\
$Y$ & 1020 & 761 & $I$ & 759 \\
$J$ & 1250 & 932 & $\cdots$ & $\cdots$ \\
$H$ & 1635 & 1220 & $J$ & 1260 \\
\hline
\end{tabular}

are consistent with $\mathrm{Xu}$ et al. (2013). A similar fit to the $\mathrm{Z}$ band did not produce a convincing fit (the reduced $\chi^{2} /$ d.o.f. was 5.44) and so we do not have confidence in the peak time for that band.

Moreover, Figure 9 also compares our residuals with the P60 (Perley et al. 2014), NOT (Xu et al. 2013) and VLT (Melandri et al. 2014) photometry. This shows the superior temporal coverage of the RATIR data (we have photometry for every night from nights 5 to 40) and the lower noise. For example, the errors (associated with the observations) in our residuals, calculated around the SN peak in $r$-band (between 18 and 26 days after the GRB trigger) are around $0.48 \mu \mathrm{Jy}$, while Perley et al. (2014), Xu et al. (2013), and Melandri et al. (2014) give estimated errors of $1.7,1.5$, and $0.7 \mu \mathrm{Jy}$ for their photometry with P60, NOT, and VLT, respectively.

We determined the peak flux density and magnitudes of SN 2013cq and SN 1998bw shifted to $z=0.34$, by averaging the residuals from days 18 to 26 . These are given in Table 5. The SEDs at the peak flux of both supernovae are shown in Figure 10. Within our considerable uncertainties, the broadband SED of SN 2013cq is compatible with that of SN 1998bw for 300-800 nm and suggest similarities in the ejected ${ }^{56} \mathrm{Ni}$ masses and kinetic energies between both SNe. For SN 2013cq the rest-frame $M_{r}$ magnitude (from our observed $Z$ magnitude) is $M_{r}=-18.43 \pm 0.11$ for SN 2013cq, which is close to the measured $M_{r}=-18.48 \pm 0.08$ for SN 1998bw.

\section{SUMMARY}

We have presented griZYJH photometry of the afterglow of GRB 130427A with the RATIR instrument from the night of the burst to 3 years later. Comparing our work to previous photometric studies (Perley et al. 2014; $\mathrm{Xu}$ et al. 2013; Melandri et al. 2014), we have better temporal sampling, deeper photometry, and we subtract a deep late-epoch image to remove the host galaxy.

Perley et al. (2014) were better able to study the afterglow over the first couple of days and show the existence of a temporal break at about 0.7 days. Our data, on the other hand, are better suited to looking for deviations from simple power-law model at later times associated with a supernova.

We fit the early afterglow (up to 0.7 days) and late afterglow (after 0.7 days) with power-laws. Prior to fitting for the late afterglow, we subtracted a late image to remove the contamination from the host galaxy. The temporal index of the power-law components changes from 0.97 during the early afterglow to 1.41 during the late afterglow, in agreement with the values and temporal break 

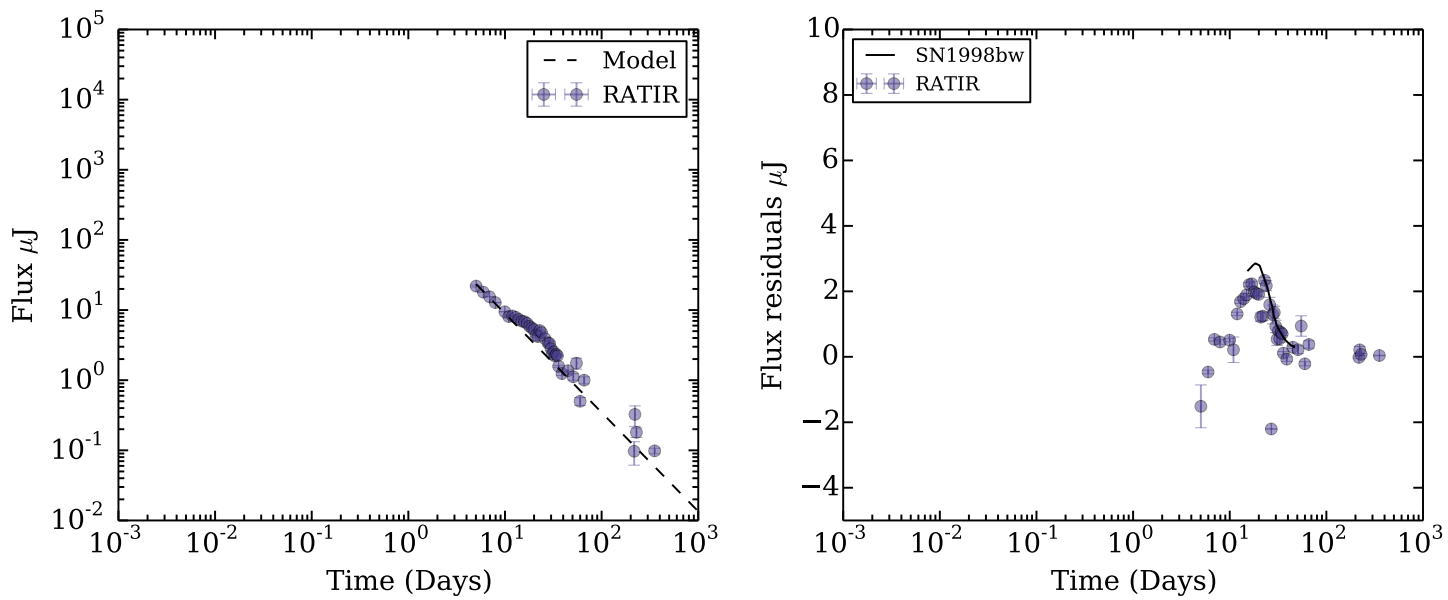

FIG. 2. - Left: The host-subtracted data (points) and power-law model (dashed line) in $g$ filter of RATIR (points). Right: Flux density residuals in $g$ (points) and the flux density of SN 1998bw in $U$ shifted to $z=0.34$ (continuous line).
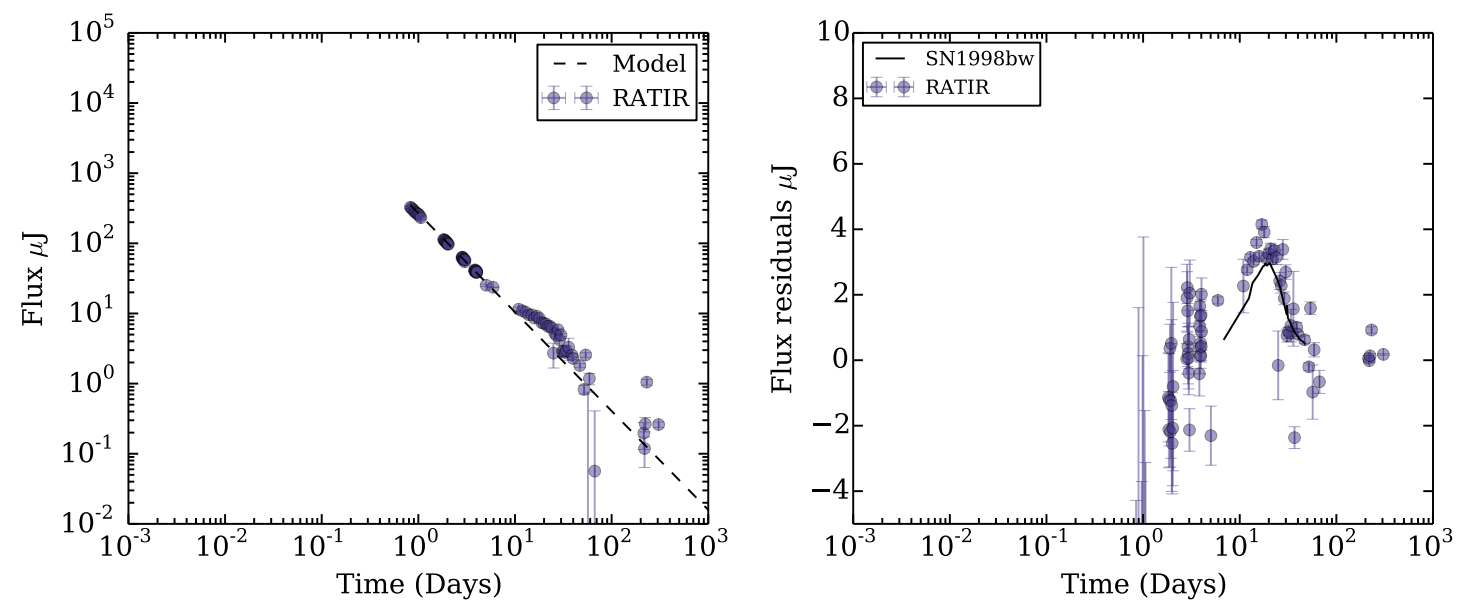

FIG. 3.- Left: The host-subtracted data (points) and power-law model (dashed line) in $r$ filter of RATIR (points). Right: Flux density residuals in $r$ (points) and the flux density of SN 1998bw in $B$ shifted to $z=0.34$ (continuous line).

TABLE 5

Peak flux Densities ANd ABSOlute Magnitudes For SN 2013CQ AND SN 1998BW SHIFTED TO $z=0.34$

\begin{tabular}{lcccc}
\hline \hline Band & \multicolumn{2}{c}{ SN 2013cq } & \multicolumn{2}{c}{ SN 1998bw at $z=0.34$} \\
& $F_{\nu}[\mu \mathrm{Jy}]$ & $M$ & $F_{\nu}[\mu \mathrm{Jy}]$ & $M$ \\
\hline$g$ & $+1.77 \pm 0.12$ & $-17.97 \pm 0.07$ & $2.58 \pm 0.07$ & $-18.38 \pm 0.03$ \\
$r$ & $+2.70 \pm 0.28$ & $-18.43 \pm 0.11$ & $2.82 \pm 0.08$ & $-18.48 \pm 0.03$ \\
$i$ & $+3.59 \pm 0.23$ & $-18.74 \pm 0.07$ & $3.15 \pm 0.09$ & $-18.60 \pm 0.03$ \\
$Z$ & $+2.80 \pm 0.87$ & $-18.47 \pm 0.33$ & $2.70 \pm 0.07$ & $-18.43 \pm 0.03$ \\
$Y$ & $+3.30 \pm 1.86$ & $-18.65 \pm 0.61$ & $\cdots$ & $\cdots$ \\
$J$ & $+2.28 \pm 2.69$ & $-18.24 \pm 0.28$ & $3.57 \pm 0.10$ & $-18.74 \pm 0.03$ \\
$H$ & $\cdots$ & $\cdots$ & $\cdots$ & $\cdots$ \\
\hline
\end{tabular}



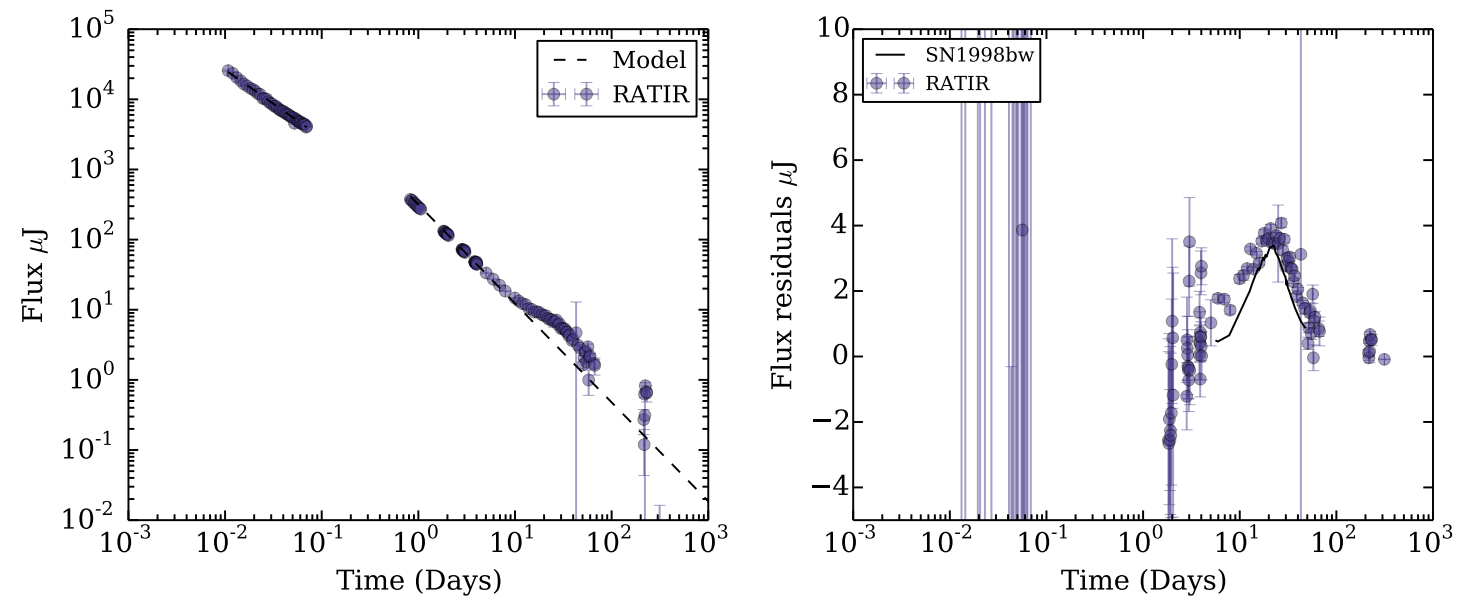

FIG. 4. - Left: The host-subtracted data (points) and power-law model (dashed line) in $i$ filter of RATIR (points). Right: Flux density residuals in $i$ (points) and the flux density of SN 1998bw in $V$ shifted to $z=0.34$ (continuous line).
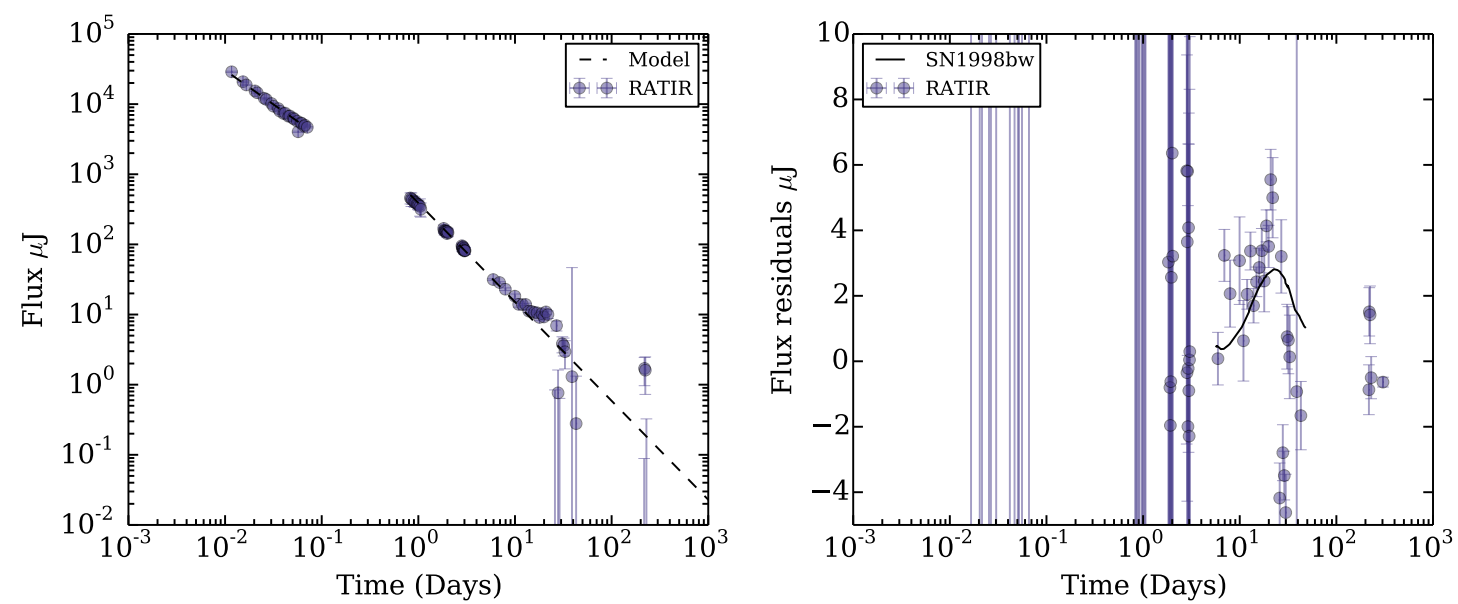

FIG. 5.- Left: The host-subtracted data (points) and power-law model (dashed line) in $Z$ filter of RATIR (points). Right: Flux density residuals in $Z$ (points) and the flux density of SN 1998bw in $R$ shifted to $z=0.34$ (continuous line). 

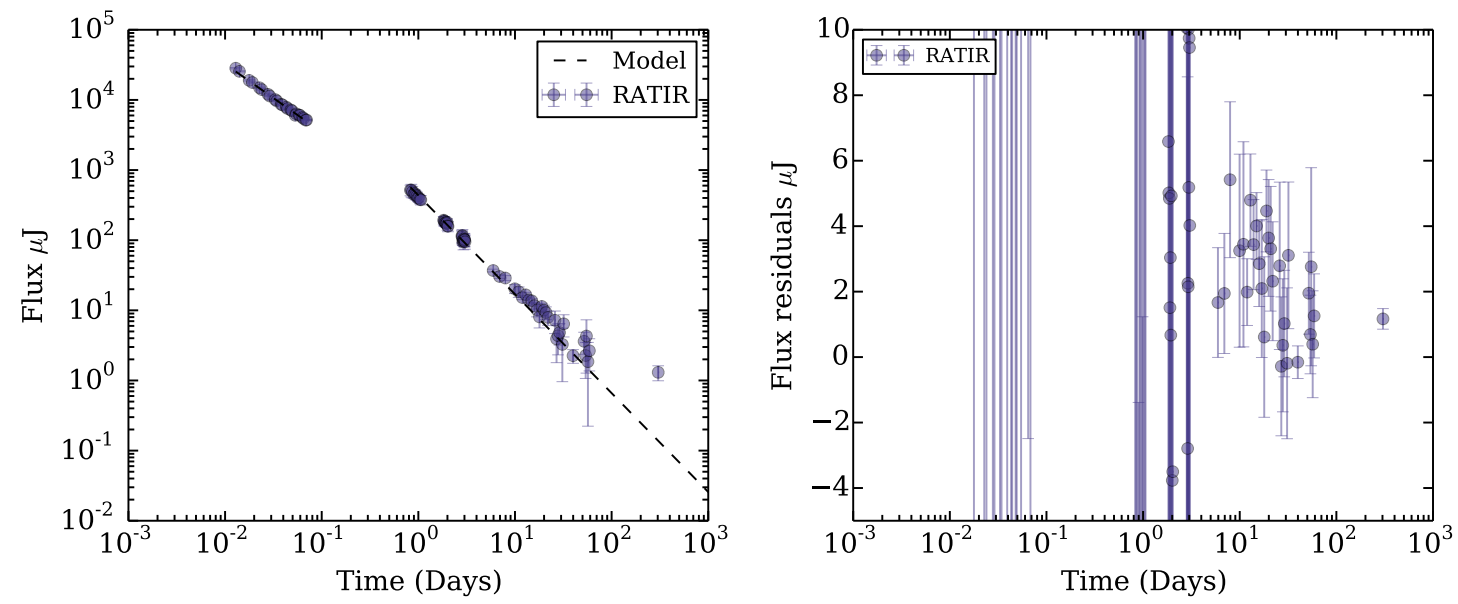

FIG. 6. - Left: The host-subtracted data (points) power-law model (dashed line) in $Y$ filter of RATIR. Right: Flux density residuals in $Y$ (points).
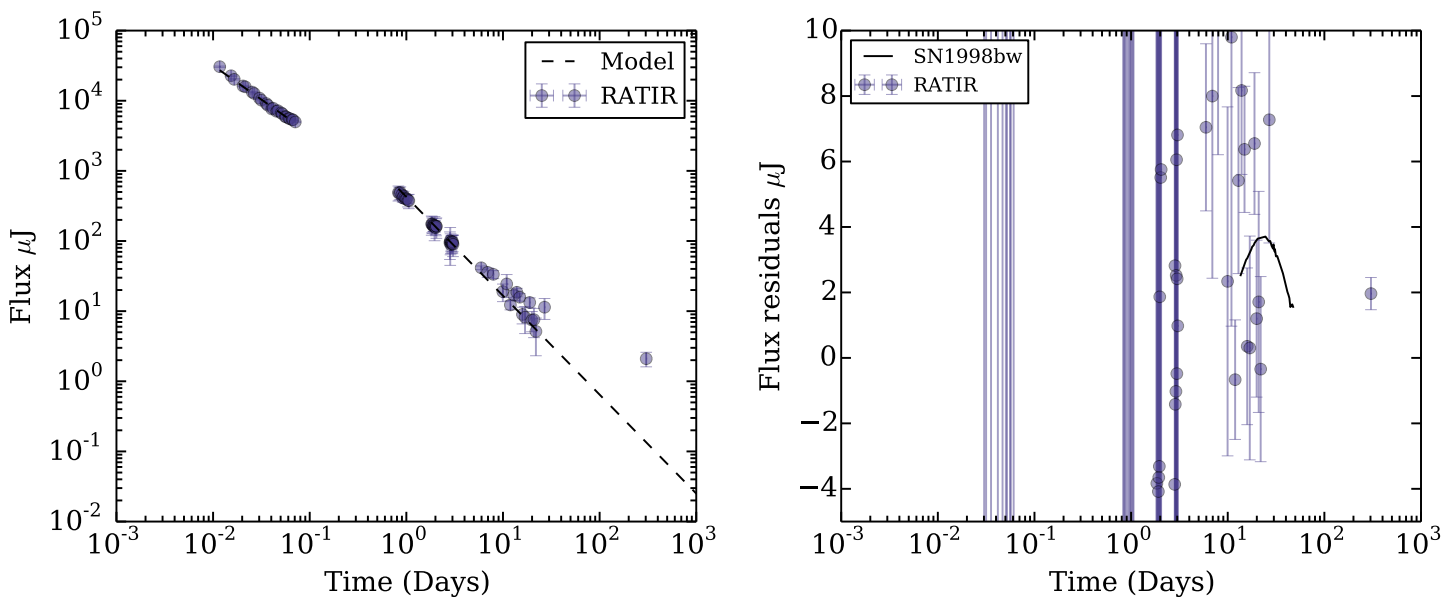

FIG. 7.- Left: The host-subtracted data (points) and power-law model (dashed line) in $J$ filter of RATIR. Right: Flux density residuals in $J$ (points) and the flux density of SN 1998bw in $I$ shifted to $z=0.34$ (continuous line).

around 0.7 days determined by Maselli et al. (2014) and Perley et al. (2014).

Positive residuals to the fits in griZ between about 7 and 40 days show that we are seeing the photometric signature of SN 2013cq, previously detected spectroscopically by de Ugarte Postigo et al. (2013) and Xu et al. (2013) and photometrically by Perley et al. (2014), Xu et al. (2013), and Melandri et al. (2014). The absolute magnitude and broadband SED of the supernova are consistent with those of the prototype SN 1998bw and suggest similar progenitors. The peak times agree with the reported by Xu et al. (2013) and is detailed for griz bands. The absolute magnitudes calculated match with SN 1998bw for riz bands. Our better temporal coverage and deeper photometry give us an improved light curve compared to previous work (Xu et al. 2013; Perley et al. 2014; Melandri et al. 2014). Photometric data obtained three years after the GRB 130427A suggest that the host galaxy is extremely blue compared to local samples.

GRB 130427A is among the handful of events with a confirmed GRB/SN association. In addition it is a highluminosity event, differing from sub-luminous, very local ones such as GRB980425/SN1998bw. The combination is thus unique, and the detailed, homogeneous photometry here presented aims to enlarge the sample for which detailed inferences can be made, eventually in a more statistically significant way when combined with other bursts. Then, the photometry here presented aims to enlarge the sample for which detailed inferences can be made, eventually in a more statistically significant way 

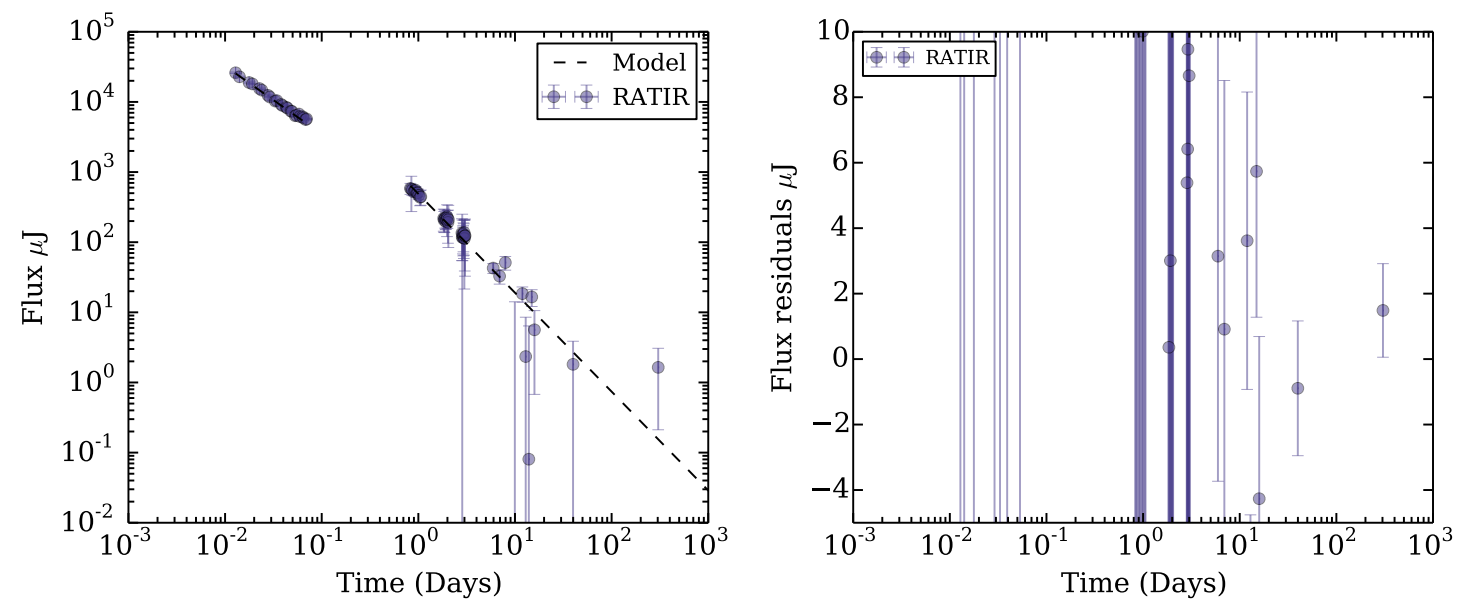

FIG. 8. - Left: The host-subtracted data (points) and power-law model (dashed line) in $H$ filter of RATIR. Right: Flux density residuals in $H$ (points).

when combined with other bursts.

\section{ACKNOWLEDGMENTS}

We thank the staff of the Observatorio Astronómico Nacional on Sierra San Pedro Mártir. We thank the anonymous referee for a very helpful report. We thank Fabio De Colle for useful comments on earlier drafts. RATIR is a collaboration between the University of California, the Universidad Nacional Autonóma de México, NASA Goddard Space Flight Center, and Arizona State
University, benefiting from the loan of an H2RG detector and hardware and software support from Teledyne Scientific and Imaging. RATIR, the automation of the Harold L. Johnson Telescope of the Observatorio Astronómico Nacional on Sierra San Pedro Mártir, and the operation of both are funded through NASA grants NNX09AH71G, NNX09AT02G, NNX10AI27G, and NNX12AE66G, CONACyT grants INFR-2009-01122785 and CB-2008-101958, UNAM PAPIIT grants IG100414 and IA102917, UC MEXUS-CONACyT grant CN 09-283, and the Instituto de Astronomía of the Universidad Nacional Autonóma de México.

\section{REFERENCES}

Ackermann, M., Ajello, M., Asano, K., et al. 2014, Science, 343 42

Arnett, W. D. 1982, ApJ, 253, 785

Barthelmy, S. D., Baumgartner, W. H., Cummings, J. R., et al. 2013, GRB Coordinates Network, 14470, 1

Becker, A. 2015, Astrophysics Source Code Library, ascl:1504.004

Berger, E., Fox, D. B., Cucchiara, A., \& Cenko, S. B. 2008, GRB Coordinates Network, 8335, 1

Berger, E., Chornock, R., Holmes, T. R., et al. 2011, ApJ, 743, 204

Bersier, D. 2012, arXiv:1206.6979

Bloom, J. S., Kulkarni, S. R., Djorgovski, S. G., et al. 1999, Nature, 401, 453

Bloom, J. S., Kulkarni, S. R., Price, P. A., et al. 2002, ApJ, 572, L45

Bufano, F., Pian, E., Sollerman, J., et al. 2012, ApJ, 753, 67

Butler, N., Klein, C., Fox, O., et al. 2012, Proc. SPIE, 8446, 844610

Butler, N., Watson, A. M., Kutyrev, A., et al. 2013, GRB Coordinates Network, 14459, 1

Butler, N., Watson, A. M., Kutyrev, A., et al. 2013, GRB Coordinates Network, 14483, 1

Butler, N., Watson, A. M., Kutyrev, A., et al. 2013, GRB Coordinates Network, 14506, 1

Butler, N., Watson, A. M., Kutyrev, A., et al. 2013, GRB Coordinates Network, 14514, 1

Watson, A. M., Butler, N., Kutyrev, A., et al. 2013, GRB Coordinates Network, 14606, 1

Watson, A. M., Butler, N., Kutyrev, A., et al. 2013, GRB Coordinates Network, 14666, 1
Campana, S., Mangano, V., Blustin, A. J., et al. 2006, Nature, 442, 1008

Cano, Z., Bersier, D., Guidorzi, C., et al. 2011, ApJ, 740, 41

Cano, Z., de Ugarte Postigo, A., Perley, D., et al. 2015, MNRAS, 452,1535

Chevalier, R. A., \& Li, Z.-Y. 2000, ApJ, 536, 195

Chornock, R., Berger, E., Levesque, E. M., et al. 2010, arXiv:1004.2262

Clocchiatti, A., Suntzeff, N. B., Covarrubias, R., \& Candia, P. 2011, AJ, 141, 163

Cobb, B. E., Bloom, J. S., Perley, D. A., et al. 2010, ApJ, 718, L150

de Ugarte Postigo, A., Xu, D., Leloudas, G., et al. 2013, GRB Coordinates Network, 14646, 1

D'Elia, V., Pian, E., Melandri, A., et al. 2015, A\&A, 577, A116

Della Valle, M., Malesani, D., Benetti, S., et al. 2003, A\&A, 406, L33

Della Valle, M., Malesani, D., Bloom, J. S., et al. 2006, ApJ, 642, L103

Della Valle, M., Benetti, S., Mazzali, P., et al. 2008, Central Bureau Electronic Telegrams, 1602, 1

Drout, M. R., Soderberg, A. M., Gal-Yam, A., et al. 2011, ApJ, 741,97

Eichler, D., Livio, M., Piran, T., \& Schramm, D. N. 1989, Nature, 340,126

Ferrero, P., Kann, D. A., Zeh, A., et al. 2006, A\&A, 457, 857

Flores, H., Covino, S., Xu, D., et al. 2013, GRB Coordinates Network, 14491, 1

Foley, S., Watson, D., Gorosabel, J., et al. 2006, A\&A, 447, 891

Fox, O. D., Kutyrev, A. S., Rapchun, D. A., et al. 2012,

Proc. SPIE, 8453, 84531O 

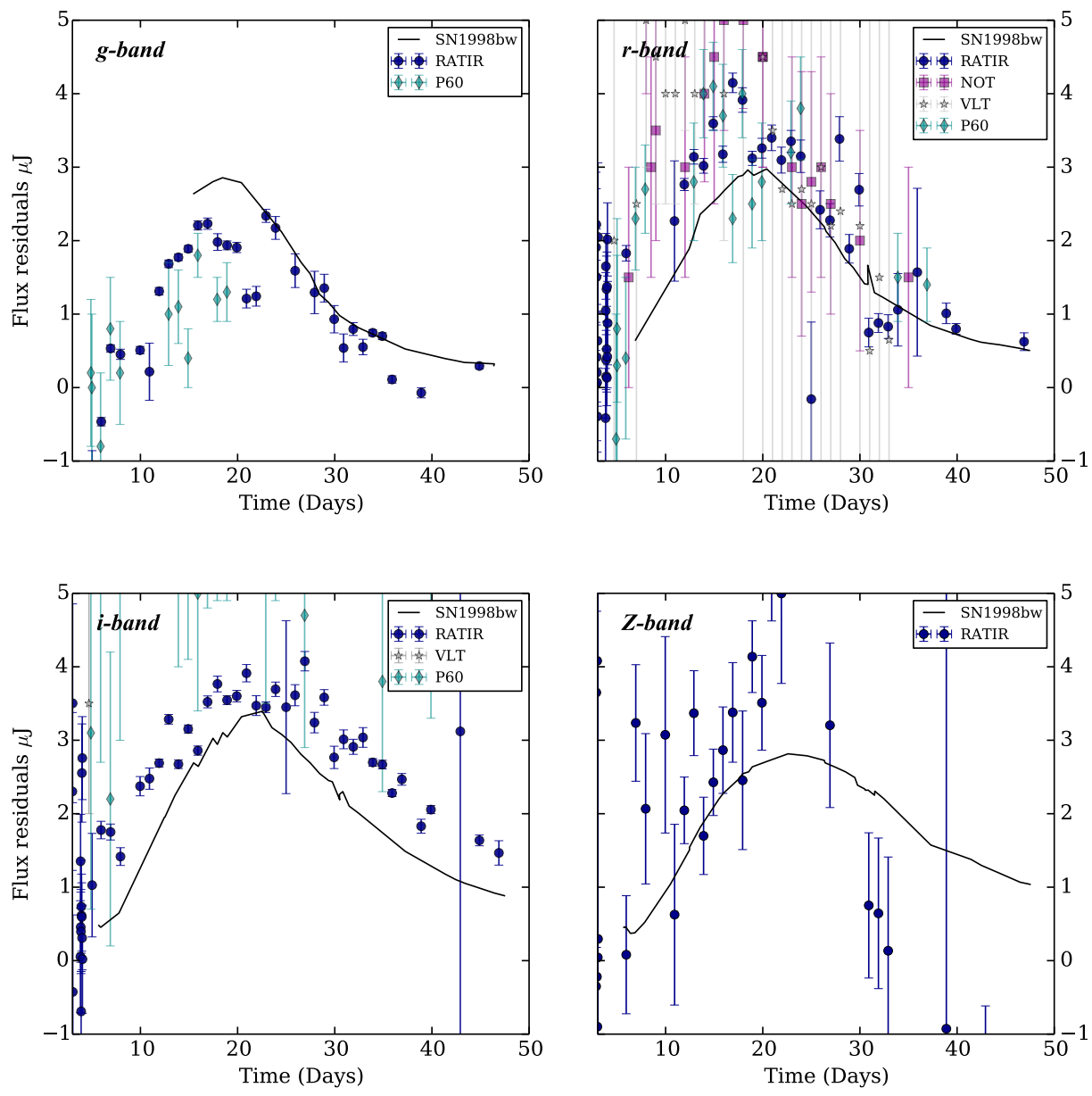

FIG. 9.- Light curves of SN 2013cq associated with GRB 130427A in the griZ bands. RATIR from this work (points), P60 from Perley et al. (2014) (diamonds), and NOT from (Xu et al. 2013) (squares), and VLT from (Melandri et al. 2014) (stars). The line shows the light curves for SN 1998bw are shifted to $z=0.34$.

Fraija, N. 2015, ApJ, 804, 105

Fraija, N., Lee, W., \& Veres, P. 2016, ApJ, 818, 190

Fraija, N., Lee, W., Veres, P. \& Barniol Duran, R. 2016, ApJ, 831,22

Fruchter, A.S. et al., 2006, Nature, 441, 463

Fynbo, J. U., Holland, S., Andersen, M. I., et al. 2000, ApJ, 542, L89

Gal-Yam, A., Moon, D.-S., Fox, D. B., et al. 2004, ApJ, 609, L59

Galama, T. J., Vreeswijk, P. M., van Paradijs, J., et al. 1998, Nature, 395, 670

Gao, H., Lei, W.-H., Zou, Y.-C., Wu, X.-F., \& Zhang, B. 2013, New Astronomy Reviews, 57, 141

Garnavich, P. M., Stanek, K. Z., Wyrzykowski, L., et al. 2003, ApJ, 582, 924

Gavazzi, G., Fumagalli, M., Cucciati, O., \& Boselli, A. 2010, A\&A, 517, A73

Gehrels, N., \& Razzaque, S. 2013, Frontiers of Physics, 8, 661 González Delgado, R. M., Cid Fernandes, R., Pérez, E., et al. 2016, A\&A, 590, A44

Granot, J., \& Sari, R. 2002, ApJ, 568, 820

Greiner, J., Klose, S., Reinsch, K., et al. 2003, Nature, 426, 157

Hammer, F., Flores, H., Schaerer, D., et al. 2006, A\&A, 454, 103
Hjorth, J., \& Bloom, J. S. 2012, Chapter 9 in "Gamma-Ray Bursts", Cambridge Astrophysics Series 51, eds. C. Kouveliotou, R. A. M. J. Wijers and S. Woosley, Cambridge University Press (Cambridge), p. 169-190, 169

Hjorth, J. 2013, Philosophical Transactions of the Royal Society of London Series A, 371, 20120275

Iwamoto, K., Nomoto, K., Mazzali, P. A., \& et al. 2003, Supernovae and Gamma-Ray Bursters, 598, 243

Koshut, T. M., Paciesas, W. S., Kouveliotou, C., et al. 1995, Bulletin of the American Astronomical Society, 27, \#53.01

Kouveliotou, C., Granot, J., Racusin, J. L., et al. 2013, ApJ, 779, L1

Kumar, P., \& Zhang, B. 2015, Phys. Rep., 561,

Kumar, P., \& Piran, T. 2000, ApJ, 532, 286

Kouveliotou, C., Meegan, C. A., Fishman, G. J., Bhat, N. P.,

Briggs, M. S., Koshut, T. M., Paciesas, W. S., \& Pendleton, G. N. 1993, ApJ, 413, L101

Lang, D., Hogg, D. W., Mierle, K., Blanton, M., \& Roweis, S. 2010, AJ, 139, 1782

Laskar, T., Berger, E., Zauderer, B. A., et al. 2013, ApJ, 776, 119

Lattimer, J. M., \& Schramm, D. N. 1976, ApJ, 210, 549

Lee, W. H., \& Ramirez-Ruiz, E. 2007, New Journal of Physics, 9, 17 


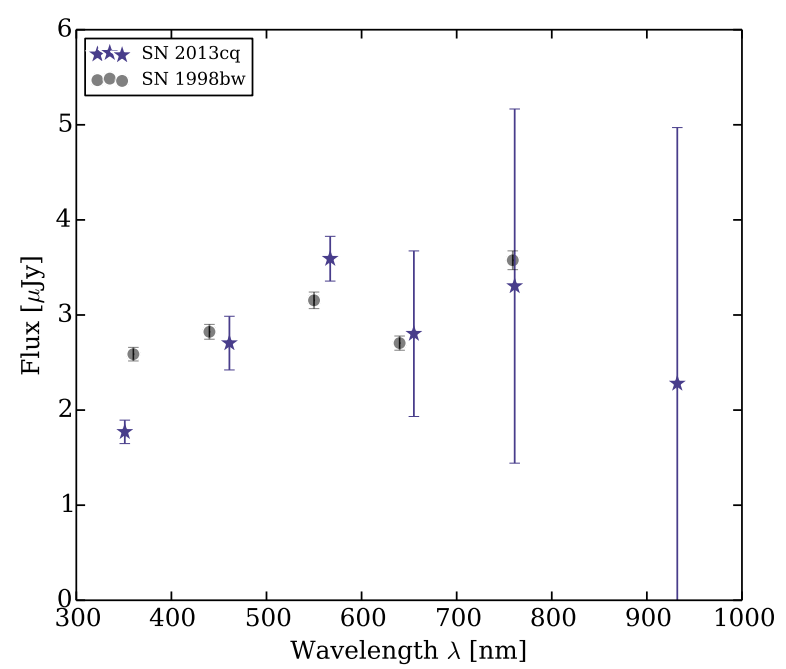

FIG. 10. - Broad-band rest-frame SED of SN 2013cq (stars) and SN 1998bw (circles) by averaging their fluxes from days 18 to 26 . The wavelength is in the rest frame, but the flux density is observed for SN 2013cq and shifted to $z=0.34$ for SN 1998bw.

Levan, A. J., Cenko, S. B., Perley, D. A., \& Tanvir, N. R. 2013, GRB Coordinates Network, 14455, 1

Levan, A. J., Fruchter, A. S., Graham, J., et al. 2013, GRB Coordinates Network, 14686, 1

Levan, A. J., Tanvir, N. R., Fruchter, A. S., et al. 2014, ApJ, 792, 115

Li, C., Kauffmann, G., Jing, Y. P., et al. 2006, MNRAS, 368, 21

Li, L.-X. 2006, MNRAS, 372, 1357

Littlejohns, O. M., Butler, N. R., Cucchiara, A., et al. 2015, MNRAS, 449, 2919

MacFadyen, A. I., \& Woosley, S. E. 1999, ApJ, 524, 262

Malesani, D., Tagliaferri, G., Chincarini, G., et al. 2004, ApJ, 609, L5

Maselli, A., Beardmore, A. P., Lien, A. Y., et al. 2013, GRB Coordinates Network, 14448, 1

Maselli, A., Melandri, A., Nava, L., et al. 2014, Science, 343, 48

Matheson, T., Garnavich, P. M., Stanek, K. Z., et al. 2003, ApJ, 599,394

Melandri, A., Pian, E., Ferrero, P., et al. 2012, A\&A, 547, A82

Melandri, A., Pian, E., D'Elia, V., et al. 2014, A\&A, 567, A29

Metzger, M. R., et al. 1997, Nature, 387, 878

Meszaros, P., \& Rees, M. J. 1994, MNRAS, 269, L41

Mészáros, P., \& Rees, M. J. 1997, ApJ, 476, 232

Mirabal, N., Halpern, J. P., An, D., Thorstensen, J. R., \& Terndrup, D. M. 2006, ApJ, 643, L99

Modjaz, M., Stanek, K. Z., Garnavich, P. M., et al. 2006, ApJ, 645, L21

Modjaz, M., Kewley, L., Kirshner, R. P., et al. 2008, AJ, 135, 1136

Modjaz, M. 2011, Astronomische Nachrichten, 332, 434

Nakar, E. 2007, Phys. Rep., 442, 166

Narayan, R., Paczynski, B., \& Piran, T. 1992, ApJ, 395, L83

Nakar, E., \& Piran, T. 2004, MNRAS, 353, 647

Niino, Y. 2013, EAS Publications Series, 61, 427

Nomoto, K., Tominaga, N., Tanaka, M., et al. 2006, Nuovo Cimento B Serie, 121, 1207

Olivares E., F., Greiner, J., Schady, P., et al. 2012, A\&A, 539, A76
Paczynski, B., 1989, ApJ, 308, L43

Paczynski, B., 1991, Acta Astron., 41, 257

Paczynski, B., \& Rhoads, J. E. 1993, ApJ, 418, L5

Panaitescu, A., \& Mészáros, P. 1998, ApJ, 493, L31

Panaitescu, A. 2007, MNRAS, 379, 331

Perley, D. A., Cenko, S. B., Corsi, A., et al. 2014, ApJ, 781, 37

Perley, D. A. 2013, GRB Coordinates Network, 14451, 1

Perley, D. A., \& Tang, S. 2013, GRB Coordinates Network, 14615, 1

Piran, T., \& Granot, J. 2001, Gamma-ray Bursts in the Afterglow Era, 300

Pian, E., Mazzali, P. A., Masetti, N., et al. 2006, Nature, 442, 1011

Planck Collaboration, Ade, P. A. R., Aghanim, N., et al. 2014, A\&A, 571, A1

Rees, M. J., \& Meszaros, P. 1992, MNRAS, 258, 41P

Rees, M. J., \& Meszaros, P. 1994, ApJ, 430, L93

Sari, R., Piran, T.,

Sari, R., \& Piran, T. 1999, ApJ, 520, 641 \& Narayan, R. 1998, ApJ, 497, L17

—. 1999, ApJ, 517, L109

Savaglio, S., Glazebrook, K., \& Le Borgne, D. 2009, ApJ, 691, 182

Schulze, S., Malesani, D., Cucchiara, A., et al. 2014, A\&A, 566, A102

Skibba, R. A., \& Sheth, R. K. 2009, MNRAS, 392, 1080

Soderberg, A. M., Kulkarni, S. R., Fox, D. B., et al. 2005, ApJ, 627,877

Soderberg, A., Berger, E., \& Fox, D. 2008, GRB Coordinates Network, 8662, 1

Sokolov, V. V., Castro-Tirado, A. J., Moskvitin, A. S., et al. 2013, GRB Coordinates Network, 14669, 1

Sollerman, J., Jaunsen, A. O., Fynbo, J. P. U., et al. 2006, A\&A, 454,503

Stanek, K. Z., Matheson, T., Garnavich, P. M., et al. 2003, ApJ, 591, L17

Starling, R. L. C., Wiersema, K., Levan, A. J., et al. 2011, MNRAS, 411, 2792

Tam, P.-H. T., Tang, Q.-W., Hou, S.-J., Liu, R.-Y., \& Wang, X.-Y. 2013, ApJ, 771, L13

Thomsen, B., Hjorth, J., Watson, D., et al. 2004, A\&A, 419, L21

Tinney, C., Stathakis, R., Cannon, R., et al. 1998, IAU Circ., 6896,1

Tominaga, N., Maeda, K., Umeda, H., et al. 2007, ApJ, 657, L77

Toy, V. L., Cenko, S. B., Silverman, J. M., et al. 2015 , arXiv:1508.00575

Vestrand, W. T., Wren, J. A., Panaitescu, A., et al. 2014, Science, 343,38

van der Horst, A. J., Paragi, Z., de Bruyn, A. G., et al. 2014, MNRAS, 444, 3151

von Kienlin, A. 2013, GRB Coordinates Network, 14473, 1

Volnova, A., Elenin, L., \& Pozanenko, A. 2013, GRB Coordinates Network, 14516, 1

Watson, A. M., Richer, M. G., Bloom, J. S., et al. 2012 , Proc. SPIE, 8444, 84445L

Watson, A. M., Butler, N., Kutyrev, A., et al. 2013, GRB

Coordinates Network, 14666, 1

Wiersema, K., Vaduvescu, O., Tanvir, N., Levan, A., \& Hartoog, O. 2013, GRB Coordinates Network, 14617, 1

Woosley, S. E., \& Bloom, J. S. 2006, ARA\&A, 44, 507

Woosley, S. E. 1993, ApJ, 405, 273

Woosley, S. E., \& Bloom, J. S. 2006, ARA\&A, 44, 507

$\mathrm{Xu}$, D., de Ugarte Postigo, A., Schulze, S., et al. 2013, GRB Coordinates Network, 14478, 1

Xu, D., de Ugarte Postigo, A., Leloudas, G., et al. 2013, ApJ, 776,98

Zeh, A., Klose, S., \& Hartmann, D. H. 2004, ApJ, 609, 952 
TABLE 6

Photometry of GRB 130427A

\begin{tabular}{|c|c|c|c|c|c|c|}
\hline$t_{0}(\mathrm{~d})$ & $t_{0}(\mathrm{~s})$ & $t_{e}(\mathrm{~s})$ & $t_{f}(\mathrm{~s})$ & Filter & $\begin{array}{c}m(\mathrm{AB}) \\
\text { (aperture) }\end{array}$ & $\begin{array}{c}\text { Flux }(\mu \mathrm{Jy}) \\
(\mathrm{PSF})\end{array}$ \\
\hline 0.01 & 930 & 80 & 0.01 & $i$ & $12.88 \pm 0.02$ & \\
\hline 0.01 & 1009 & 67 & 0.01 & $Z$ & $12.75 \pm 0.02$ & \\
\hline 0.01 & 1012 & 67 & 0.01 & $J$ & $12.69 \pm 0.02$ & \\
\hline 0.01 & 1037 & 80 & 0.01 & $i$ & $12.97 \pm 0.02$ & \\
\hline 0.01 & 1111 & 67 & 0.01 & $H$ & $12.86 \pm 0.02$ & \\
\hline 0.01 & 1114 & 67 & 0.01 & $Y$ & $12.77 \pm 0.02$ & \\
\hline 0.01 & 1141 & 80 & 0.01 & $i$ & $13.12 \pm 0.02$ & \\
\hline 0.01 & 1216 & 67 & 0.01 & $Y$ & $12.88 \pm 0.02$ & \\
\hline 0.01 & 1216 & 67 & 0.01 & $H$ & $13.00 \pm 0.02$ & \\
\hline 0.01 & 1249 & 80 & 0.02 & $i$ & $13.24 \pm 0.02$ & \\
\hline 0.02 & 1323 & 67 & 0.02 & $Z$ & $13.10 \pm 0.02$ & \\
\hline 0.02 & 1326 & 67 & 0.02 & $J$ & $13.01 \pm 0.02$ & \\
\hline 0.02 & 1348 & 80 & 0.02 & $i$ & $13.35 \pm 0.02$ & \\
\hline 0.02 & 1429 & 67 & 0.02 & $J$ & $13.14 \pm 0.02$ & \\
\hline 0.02 & 1432 & 67 & 0.02 & $Z$ & $13.22 \pm 0.02$ & \\
\hline 0.02 & 1458 & 80 & 0.02 & $i$ & $13.42 \pm 0.02$ & \\
\hline 0.02 & 1532 & 67 & 0.02 & $H$ & $13.21 \pm 0.03$ & \\
\hline 0.02 & 1534 & 67 & 0.02 & $Y$ & $13.20 \pm 0.02$ & \\
\hline 0.02 & 1579 & 80 & 0.02 & $i$ & $13.51 \pm 0.02$ & \\
\hline 0.02 & 1653 & 67 & 0.02 & $H$ & $13.26 \pm 0.02$ & \\
\hline 0.02 & 1653 & 67 & 0.02 & $Y$ & $13.28 \pm 0.02$ & \\
\hline 0.02 & 1684 & 80 & 0.02 & $i$ & $13.55 \pm 0.02$ & \\
\hline 0.02 & 1762 & 67 & 0.02 & $Z$ & $13.43 \pm 0.02$ & \\
\hline 0.02 & 1764 & 67 & 0.02 & $J$ & $13.37 \pm 0.02$ & \\
\hline 0.02 & 1778 & 80 & 0.02 & $i$ & $13.61 \pm 0.02$ & \\
\hline 0.02 & 1858 & 67 & 0.02 & $J$ & $13.40 \pm 0.02$ & \\
\hline 0.02 & 1859 & 67 & 0.02 & $Z$ & $13.50 \pm 0.02$ & \\
\hline 0.02 & 1888 & 80 & 0.02 & $i$ & $13.70 \pm 0.02$ & \\
\hline 0.02 & 1962 & 67 & 0.02 & $Y$ & $13.47 \pm 0.02$ & \\
\hline 0.02 & 1964 & 67 & 0.02 & $H$ & $13.43 \pm 0.03$ & \\
\hline 0.02 & 1993 & 80 & 0.02 & $i$ & $13.72 \pm 0.02$ & \\
\hline 0.02 & 2067 & 67 & 0.02 & $Y$ & $13.53 \pm 0.02$ & \\
\hline 0.02 & 2068 & 67 & 0.02 & $H$ & $13.48 \pm 0.03$ & \\
\hline 0.02 & 2121 & 80 & 0.03 & $i$ & $13.87 \pm 0.02$ & \\
\hline 0.03 & 2196 & 67 & 0.03 & $J$ & $13.59 \pm 0.02$ & \\
\hline 0.03 & 2196 & 67 & 0.03 & $Z$ & $13.69 \pm 0.02$ & \\
\hline 0.03 & 2224 & 80 & 0.03 & $i$ & $13.87 \pm 0.02$ & \\
\hline 0.03 & 2298 & 67 & 0.03 & $J$ & $13.64 \pm 0.02$ & \\
\hline 0.03 & 2300 & 67 & 0.03 & $Z$ & $13.73 \pm 0.02$ & \\
\hline 0.03 & 2329 & 80 & 0.03 & $i$ & $13.89 \pm 0.02$ & \\
\hline 0.03 & 2408 & 67 & 0.03 & $H$ & $13.67 \pm 0.02$ & \\
\hline 0.03 & 2408 & 67 & 0.03 & $Y$ & $13.70 \pm 0.02$ & \\
\hline 0.03 & 2422 & 80 & 0.03 & $i$ & $13.98 \pm 0.02$ & \\
\hline 0.03 & 2502 & 67 & 0.03 & $Y$ & $13.76 \pm 0.02$ & \\
\hline 0.03 & 2506 & 67 & 0.03 & $H$ & $13.72 \pm 0.02$ & \\
\hline 0.03 & 2531 & 80 & 0.03 & $i$ & $14.03 \pm 0.02$ & \\
\hline 0.03 & 2605 & 67 & 0.03 & $J$ & $13.81 \pm 0.02$ & \\
\hline 0.03 & 2607 & 67 & 0.03 & $Z$ & $13.88 \pm 0.02$ & \\
\hline 0.03 & 2655 & 80 & 0.03 & $i$ & $14.07 \pm 0.02$ & \\
\hline 0.03 & 2729 & 67 & 0.03 & $J$ & $13.88 \pm 0.02$ & \\
\hline 0.03 & 2732 & 67 & 0.03 & $Z$ & $13.97 \pm 0.02$ & \\
\hline 0.03 & 2780 & 80 & 0.03 & $i$ & $14.15 \pm 0.02$ & \\
\hline 0.03 & 2855 & 67 & 0.03 & $Y$ & $13.89 \pm 0.02$ & \\
\hline 0.03 & 2856 & 67 & 0.03 & $H$ & $13.87 \pm 0.03$ & \\
\hline 0.03 & 2885 & 80 & 0.03 & $i$ & $14.14 \pm 0.02$ & \\
\hline 0.03 & 2960 & 67 & 0.04 & $Y$ & $13.93 \pm 0.02$ & \\
\hline 0.03 & 2961 & 67 & 0.04 & $H$ & $13.85 \pm 0.03$ & \\
\hline 0.03 & 2990 & 80 & 0.04 & $i$ & $14.19 \pm 0.02$ & \\
\hline 0.04 & 3067 & 67 & 0.04 & $Z$ & $14.06 \pm 0.02$ & \\
\hline 0.04 & 3069 & 67 & 0.04 & $J$ & $14.00 \pm 0.02$ & \\
\hline 0.04 & 3108 & 80 & 0.04 & $i$ & $14.25 \pm 0.02$ & \\
\hline 0.04 & 3186 & 67 & 0.04 & $J$ & $14.06 \pm 0.02$ & \\
\hline 0.04 & 3186 & 67 & 0.04 & $Z$ & $14.15 \pm 0.02$ & \\
\hline 0.04 & 3214 & 80 & 0.04 & $i$ & $14.28 \pm 0.02$ & \\
\hline 0.04 & 3292 & 67 & 0.04 & $Y$ & $14.06 \pm 0.02$ & \\
\hline 0.04 & 3293 & 67 & 0.04 & $H$ & $13.99 \pm 0.03$ & \\
\hline 0.04 & 3307 & 80 & 0.04 & $i$ & $14.31 \pm 0.02$ & \\
\hline 0.04 & 3387 & 67 & 0.04 & $H$ & $14.03 \pm 0.03$ & \\
\hline 0.04 & 3389 & 67 & 0.04 & $Y$ & $14.08 \pm 0.02$ & \\
\hline 0.04 & 3418 & 80 & 0.04 & $i$ & $14.32 \pm 0.02$ & \\
\hline 0.04 & 3493 & 67 & 0.04 & $J$ & $14.19 \pm 0.02$ & \\
\hline 0.04 & 3493 & 67 & 0.04 & $Z$ & $14.24 \pm 0.02$ & \\
\hline 0.04 & 3535 & 80 & 0.04 & $i$ & $14.35 \pm 0.02$ & \\
\hline 0.04 & 3610 & 67 & 0.04 & $Z$ & $14.22 \pm 0.02$ & \\
\hline 0.04 & 3611 & 67 & 0.04 & $J$ & $14.17 \pm 0.02$ & \\
\hline
\end{tabular}


TABLE 6 - Continued

\begin{tabular}{|c|c|c|c|c|c|c|}
\hline$t_{0}(\mathrm{~d})$ & $t_{0}(\mathrm{~s})$ & $t_{e}(\mathrm{~s})$ & $t_{f}(\mathrm{~s})$ & Filter & $\begin{array}{c}m(\mathrm{AB}) \\
\text { (aperture) }\end{array}$ & $\begin{array}{c}\text { Flux }(\mu \mathrm{Jy}) \\
(\mathrm{PSF})\end{array}$ \\
\hline 0.04 & 3640 & 80 & 0.04 & $i$ & $14.40 \pm 0.02$ & \\
\hline 0.04 & 3719 & 67 & 0.04 & $H$ & $14.09 \pm 0.03$ & \\
\hline 0.04 & 3721 & 67 & 0.04 & $Y$ & $14.16 \pm 0.02$ & \\
\hline 0.04 & 3736 & 80 & 0.04 & $i$ & $14.41 \pm 0.02$ & \\
\hline 0.04 & 3814 & 67 & 0.04 & $Y$ & $14.21 \pm 0.02$ & \\
\hline 0.04 & 3815 & 67 & 0.04 & $H$ & $14.12 \pm 0.03$ & \\
\hline 0.04 & 3843 & 80 & 0.05 & $i$ & $14.44 \pm 0.02$ & \\
\hline 0.05 & 3918 & 67 & 0.05 & $J$ & $14.28 \pm 0.03$ & \\
\hline 0.05 & 3919 & 67 & 0.05 & $Z$ & $14.33 \pm 0.02$ & \\
\hline 0.05 & 3964 & 80 & 0.05 & $i$ & $14.45 \pm 0.02$ & \\
\hline 0.05 & 4038 & 67 & 0.05 & $J$ & $14.25 \pm 0.02$ & \\
\hline 0.05 & 4038 & 67 & 0.05 & $Z$ & $14.32 \pm 0.02$ & \\
\hline 0.05 & 4145 & 67 & 0.05 & $H$ & $14.23 \pm 0.03$ & \\
\hline 0.05 & 4146 & 67 & 0.05 & $Y$ & $14.26 \pm 0.02$ & \\
\hline 0.05 & 4172 & 80 & 0.05 & $i$ & $14.51 \pm 0.02$ & \\
\hline 0.05 & 4247 & 67 & 0.05 & $H$ & $14.24 \pm 0.03$ & \\
\hline 0.05 & 4248 & 67 & 0.05 & $Y$ & $14.28 \pm 0.02$ & \\
\hline 0.05 & 4295 & 80 & 0.05 & $i$ & $14.55 \pm 0.02$ & \\
\hline 0.05 & 4371 & 67 & 0.05 & $J$ & $14.33 \pm 0.02$ & \\
\hline 0.05 & 4371 & 67 & 0.05 & $Z$ & $14.40 \pm 0.02$ & \\
\hline 0.05 & 4415 & 80 & 0.05 & $i$ & $14.56 \pm 0.02$ & \\
\hline 0.05 & 4493 & 67 & 0.05 & $J$ & $14.36 \pm 0.02$ & \\
\hline 0.05 & 4493 & 67 & 0.05 & $Z$ & $14.44 \pm 0.02$ & \\
\hline 0.05 & 4523 & 80 & 0.05 & $i$ & $14.76 \pm 0.03$ & \\
\hline 0.05 & 4598 & 67 & 0.05 & $H$ & $14.39 \pm 0.07$ & \\
\hline 0.05 & 4598 & 67 & 0.05 & $Y$ & $14.45 \pm 0.03$ & \\
\hline 0.05 & 4643 & 80 & 0.05 & $i$ & $14.59 \pm 0.02$ & \\
\hline 0.05 & 4718 & 67 & 0.06 & $Y$ & $14.40 \pm 0.02$ & \\
\hline 0.05 & 4719 & 67 & 0.06 & $H$ & $14.38 \pm 0.03$ & \\
\hline 0.05 & 4742 & 80 & 0.06 & $i$ & $14.64 \pm 0.02$ & \\
\hline 0.06 & 4821 & 67 & 0.06 & $J$ & $14.46 \pm 0.02$ & \\
\hline 0.06 & 4822 & 67 & 0.06 & $Z$ & $14.51 \pm 0.02$ & \\
\hline 0.06 & 4847 & 80 & 0.06 & $i$ & $14.66 \pm 0.02$ & \\
\hline 0.06 & 4922 & 67 & 0.06 & $J$ & $14.48 \pm 0.02$ & \\
\hline 0.06 & 4923 & 67 & 0.06 & $Z$ & $14.89 \pm 0.02$ & \\
\hline 0.06 & 4954 & 80 & 0.06 & $i$ & $14.68 \pm 0.02$ & \\
\hline 0.06 & 5028 & 67 & 0.06 & $H$ & $14.33 \pm 0.05$ & \\
\hline 0.06 & 5032 & 67 & 0.06 & $Y$ & $14.44 \pm 0.03$ & \\
\hline 0.06 & 5059 & 80 & 0.06 & $i$ & $14.70 \pm 0.02$ & \\
\hline 0.06 & 5134 & 67 & 0.06 & $H$ & $14.42 \pm 0.03$ & \\
\hline 0.06 & 5135 & 67 & 0.06 & $Y$ & $14.45 \pm 0.02$ & \\
\hline 0.06 & 5186 & 80 & 0.06 & $i$ & $14.75 \pm 0.03$ & \\
\hline 0.06 & 5260 & 67 & 0.06 & $J$ & $14.53 \pm 0.02$ & \\
\hline 0.06 & 5262 & 67 & 0.06 & $Z$ & $14.57 \pm 0.02$ & \\
\hline 0.06 & 5288 & 80 & 0.06 & $i$ & $14.71 \pm 0.02$ & \\
\hline 0.06 & 5362 & 67 & 0.06 & $J$ & $14.54 \pm 0.02$ & \\
\hline 0.06 & 5362 & 67 & 0.06 & $Z$ & $14.59 \pm 0.02$ & \\
\hline 0.06 & 5395 & 80 & 0.06 & $i$ & $14.76 \pm 0.02$ & \\
\hline 0.06 & 5470 & 67 & 0.06 & $H$ & $14.43 \pm 0.04$ & \\
\hline 0.06 & 5470 & 67 & 0.06 & $Y$ & $14.52 \pm 0.02$ & \\
\hline 0.06 & 5506 & 80 & 0.06 & $i$ & $14.79 \pm 0.02$ & \\
\hline 0.06 & 5586 & 67 & 0.07 & $H$ & $14.47 \pm 0.03$ & \\
\hline 0.06 & 5586 & 67 & 0.07 & $Y$ & $14.56 \pm 0.02$ & \\
\hline 0.06 & 5615 & 80 & 0.07 & $i$ & $14.78 \pm 0.02$ & \\
\hline 0.07 & 5690 & 67 & 0.07 & $J$ & $14.58 \pm 0.02$ & \\
\hline 0.07 & 5694 & 67 & 0.07 & $Z$ & $14.69 \pm 0.02$ & \\
\hline 0.07 & 5715 & 80 & 0.07 & $i$ & $14.79 \pm 0.02$ & \\
\hline 0.07 & 5789 & 67 & 0.07 & $J$ & $14.58 \pm 0.02$ & \\
\hline 0.07 & 5790 & 67 & 0.07 & $Z$ & $14.65 \pm 0.02$ & \\
\hline 0.07 & 5823 & 80 & 0.07 & $i$ & $14.80 \pm 0.02$ & \\
\hline 0.07 & 5897 & 67 & 0.07 & $H$ & $14.53 \pm 0.02$ & \\
\hline 0.07 & 5898 & 67 & 0.07 & $Y$ & $14.62 \pm 0.02$ & \\
\hline 0.07 & 5943 & 80 & 0.07 & $i$ & $14.89 \pm 0.03$ & \\
\hline 0.07 & 6018 & 67 & 0.07 & $Y$ & $14.61 \pm 0.02$ & \\
\hline 0.07 & 6020 & 67 & 0.07 & $H$ & $14.51 \pm 0.03$ & \\
\hline 0.07 & 6051 & 80 & 0.07 & $i$ & $14.86 \pm 0.02$ & \\
\hline 0.07 & 6124 & 67 & 0.07 & $J$ & $14.66 \pm 0.02$ & \\
\hline 0.07 & 6125 & 67 & 0.07 & $Z$ & $14.73 \pm 0.02$ & \\
\hline 0.83 & 71485 & 1280 & 0.85 & $i$ & $17.45 \pm 0.42$ & $374.99 \pm 12.58$ \\
\hline 0.83 & 71485 & 1280 & 0.85 & $r$ & $17.59 \pm 0.42$ & $327.32 \pm 13.34$ \\
\hline 0.83 & 71489 & 536 & 0.85 & $Y$ & $17.12 \pm 0.42$ & $519.07 \pm 89.32$ \\
\hline 0.83 & 71489 & 536 & 0.85 & $Z$ & $17.23 \pm 0.42$ & $459.20 \pm 82.36$ \\
\hline 0.83 & 71490 & 536 & 0.85 & $H$ & $17.01 \pm 0.42$ & $584.25 \pm 104.51$ \\
\hline 0.83 & 71490 & 536 & 0.85 & $J$ & $17.14 \pm 0.42$ & $489.86 \pm 118.33$ \\
\hline 0.85 & 73170 & 1280 & 0.87 & $i$ & $17.48 \pm 0.42$ & $360.45 \pm 12.42$ \\
\hline 0.85 & 73170 & 1280 & 0.87 & $r$ & $17.61 \pm 0.42$ & $319.00 \pm 16.78$ \\
\hline
\end{tabular}


TABLE 6 - Continued

\begin{tabular}{|c|c|c|c|c|c|c|}
\hline$t_{0}(\mathrm{~d})$ & $t_{0}(\mathrm{~s})$ & $t_{e}(\mathrm{~s})$ & $t_{f}(\mathrm{~s})$ & Filter & $\begin{array}{c}m(\mathrm{AB}) \\
\text { (aperture) }\end{array}$ & $\begin{array}{c}\text { Flux }(\mu \mathrm{Jy}) \\
(\mathrm{PSF})\end{array}$ \\
\hline 0.85 & 73171 & 536 & 0.87 & $Y$ & $17.11 \pm 0.42$ & $519.29 \pm 106.76$ \\
\hline 0.85 & 73171 & 536 & 0.87 & $Z$ & $17.26 \pm 0.42$ & $444.71 \pm 97.80$ \\
\hline 0.85 & 73173 & 536 & 0.87 & $H$ & $17.04 \pm 0.42$ & $572.18 \pm 298.77$ \\
\hline 0.85 & 73173 & 536 & 0.87 & $J$ & $17.16 \pm 0.42$ & $482.33 \pm 99.53$ \\
\hline 0.87 & 74870 & 1280 & 0.89 & $i$ & $17.50 \pm 0.42$ & $361.40 \pm 11.22$ \\
\hline 0.87 & 74870 & 1280 & 0.89 & $r$ & $17.66 \pm 0.42$ & $307.57 \pm 6.73$ \\
\hline 0.87 & 74872 & 536 & 0.89 & $H$ & $17.02 \pm 0.42$ & $556.82 \pm 94.64$ \\
\hline 0.87 & 74872 & 536 & 0.89 & $J$ & $17.19 \pm 0.42$ & $492.18 \pm 98.82$ \\
\hline 0.87 & 74873 & 536 & 0.89 & $Y$ & $17.14 \pm 0.42$ & $492.73 \pm 91.88$ \\
\hline 0.87 & 74873 & 536 & 0.89 & $Z$ & $17.29 \pm 0.42$ & $434.49 \pm 62.79$ \\
\hline 0.90 & 77485 & 1280 & 0.92 & $r$ & $17.71 \pm 0.42$ & $297.70 \pm 17.58$ \\
\hline 0.90 & 77485 & 1280 & 0.92 & $i$ & $17.56 \pm 0.42$ & $335.92 \pm 15.12$ \\
\hline 0.90 & 77490 & 536 & 0.92 & $Y$ & $17.27 \pm 0.42$ & $451.80 \pm 55.35$ \\
\hline 0.90 & 77490 & 536 & 0.92 & $Z$ & $17.32 \pm 0.42$ & $413.81 \pm 67.52$ \\
\hline 0.90 & 77491 & 536 & 0.92 & $H$ & $17.14 \pm 0.42$ & $520.21 \pm 61.26$ \\
\hline 0.90 & 77491 & 536 & 0.92 & $J$ & $17.25 \pm 0.42$ & $456.95 \pm 65.92$ \\
\hline 0.92 & 79150 & 1280 & 0.93 & $i$ & $17.59 \pm 0.42$ & $329.32 \pm 11.05$ \\
\hline 0.92 & 79150 & 1280 & 0.93 & $r$ & $17.76 \pm 0.42$ & $281.54 \pm 8.12$ \\
\hline 0.92 & 79151 & 536 & 0.93 & $Y$ & $17.28 \pm 0.42$ & $466.42 \pm 62.22$ \\
\hline 0.92 & 79151 & 536 & 0.93 & $Z$ & $17.36 \pm 0.42$ & $399.12 \pm 62.30$ \\
\hline 0.92 & 79151 & 536 & 0.93 & $H$ & $17.11 \pm 0.42$ & $539.28 \pm 76.97$ \\
\hline 0.92 & 79151 & 536 & 0.93 & $J$ & $17.33 \pm 0.42$ & $412.45 \pm 54.73$ \\
\hline 0.94 & 80826 & 1280 & 0.95 & $i$ & $17.62 \pm 0.42$ & $314.80 \pm 10.13$ \\
\hline 0.94 & 80826 & 1280 & 0.95 & $r$ & $17.77 \pm 0.42$ & $275.47 \pm 8.21$ \\
\hline 0.94 & 80827 & 536 & 0.95 & $Y$ & $17.26 \pm 0.42$ & $441.59 \pm 64.98$ \\
\hline 0.94 & 80827 & 536 & 0.95 & $Z$ & $17.41 \pm 0.42$ & $391.78 \pm 29.23$ \\
\hline 0.94 & 80828 & 536 & 0.95 & $H$ & $17.17 \pm 0.42$ & $551.47 \pm 69.19$ \\
\hline 0.94 & 80828 & 536 & 0.95 & $J$ & $17.26 \pm 0.42$ & $437.22 \pm 73.40$ \\
\hline 0.97 & 83586 & 1280 & 0.99 & $i$ & $17.65 \pm 0.42$ & $306.26 \pm 11.71$ \\
\hline 0.97 & 83586 & 1280 & 0.99 & $r$ & $17.81 \pm 0.42$ & $267.65 \pm 10.62$ \\
\hline 0.97 & 83593 & 536 & 0.99 & $H$ & $17.23 \pm 0.42$ & $504.95 \pm 57.64$ \\
\hline 0.97 & 83593 & 536 & 0.99 & $J$ & $17.35 \pm 0.42$ & $411.12 \pm 57.56$ \\
\hline 0.97 & 83632 & 536 & 0.99 & $Y$ & $17.30 \pm 0.42$ & $427.96 \pm 57.65$ \\
\hline 0.97 & 83632 & 536 & 0.99 & $Z$ & $17.42 \pm 0.42$ & $380.83 \pm 41.56$ \\
\hline 0.99 & 85331 & 1280 & 1.01 & $i$ & $17.69 \pm 0.42$ & $295.68 \pm 8.70$ \\
\hline 0.99 & 85331 & 1280 & 1.01 & $r$ & $17.84 \pm 0.42$ & $260.68 \pm 13.34$ \\
\hline 0.99 & 85333 & 536 & 1.01 & $Y$ & $17.37 \pm 0.42$ & $401.57 \pm 43.58$ \\
\hline 0.99 & 85333 & 536 & 1.01 & $Z$ & $17.43 \pm 0.42$ & $372.94 \pm 56.57$ \\
\hline 0.99 & 85333 & 536 & 1.01 & $H$ & $17.23 \pm 0.42$ & $507.92 \pm 63.59$ \\
\hline 0.99 & 85333 & 536 & 1.01 & $J$ & $17.38 \pm 0.42$ & $395.39 \pm 53.28$ \\
\hline 1.01 & 87061 & 1280 & 1.03 & $r$ & $17.84 \pm 0.42$ & $258.40 \pm 11.61$ \\
\hline 1.01 & 87061 & 1280 & 1.03 & $i$ & $17.73 \pm 0.42$ & $285.60 \pm 10.05$ \\
\hline 1.01 & 87064 & 536 & 1.03 & $Y$ & $17.40 \pm 0.42$ & $401.01 \pm 52.93$ \\
\hline 1.01 & 87064 & 536 & 1.03 & $Z$ & $17.49 \pm 0.42$ & $362.49 \pm 54.65$ \\
\hline 1.01 & 87066 & 536 & 1.03 & $H$ & $17.25 \pm 0.42$ & $466.24 \pm 60.97$ \\
\hline 1.01 & 87066 & 536 & 1.03 & $J$ & $17.38 \pm 0.42$ & $400.08 \pm 54.92$ \\
\hline 1.04 & 89861 & 1280 & 1.06 & $i$ & $17.76 \pm 0.42$ & $278.74 \pm 9.38$ \\
\hline 1.04 & 89861 & 1280 & 1.06 & $r$ & $17.90 \pm 0.42$ & $243.80 \pm 9.30$ \\
\hline 1.04 & 89867 & 536 & 1.06 & $Y$ & $17.41 \pm 0.42$ & $382.43 \pm 51.77$ \\
\hline 1.04 & 89867 & 536 & 1.06 & $Z$ & $17.50 \pm 0.42$ & $348.99 \pm 96.74$ \\
\hline 1.04 & 89871 & 536 & 1.06 & $H$ & $17.33 \pm 0.42$ & $437.23 \pm 106.18$ \\
\hline 1.04 & 89871 & 536 & 1.06 & $J$ & $17.41 \pm 0.42$ & $380.89 \pm 75.30$ \\
\hline 1.06 & 91596 & 1280 & 1.08 & $i$ & $17.78 \pm 0.42$ & $271.33 \pm 9.76$ \\
\hline 1.06 & 91596 & 1280 & 1.08 & $r$ & $17.94 \pm 0.42$ & $231.70 \pm 13.04$ \\
\hline 1.06 & 91600 & 536 & 1.08 & $H$ & $17.28 \pm 0.42$ & $445.08 \pm 108.89$ \\
\hline 1.06 & 91600 & 536 & 1.08 & $J$ & $17.45 \pm 0.42$ & $375.58 \pm 86.51$ \\
\hline 1.06 & 91600 & 536 & 1.08 & $Y$ & $17.41 \pm 0.42$ & $376.09 \pm 57.58$ \\
\hline 1.06 & 91600 & 536 & 1.08 & $Z$ & $17.58 \pm 0.42$ & $317.29 \pm 72.73$ \\
\hline 1.83 & 157967 & 1280 & 1.85 & $r$ & $18.70 \pm 0.42$ & $113.69 \pm 1.72$ \\
\hline 1.83 & 157967 & 1280 & 1.85 & $i$ & $18.53 \pm 0.42$ & $132.35 \pm 3.46$ \\
\hline 1.83 & 157972 & 536 & 1.85 & $H$ & $18.05 \pm 0.43$ & $219.71 \pm 76.44$ \\
\hline 1.83 & 157972 & 536 & 1.85 & $J$ & $18.23 \pm 0.43$ & $173.86 \pm 53.15$ \\
\hline 1.83 & 157972 & 536 & 1.85 & $Y$ & $18.08 \pm 0.42$ & $192.81 \pm 23.85$ \\
\hline 1.83 & 157972 & 536 & 1.85 & $Z$ & $18.25 \pm 0.42$ & $169.00 \pm 20.03$ \\
\hline 1.85 & 159663 & 1280 & 1.87 & $r$ & $18.70 \pm 0.42$ & $110.99 \pm 1.53$ \\
\hline 1.85 & 159663 & 1280 & 1.87 & $i$ & $18.55 \pm 0.42$ & $130.24 \pm 3.16$ \\
\hline 1.85 & 159666 & 536 & 1.87 & $H$ & $18.06 \pm 0.43$ & $206.18 \pm 67.93$ \\
\hline 1.85 & 159666 & 536 & 1.87 & $J$ & $18.21 \pm 0.43$ & $175.43 \pm 44.56$ \\
\hline 1.85 & 159668 & 536 & 1.87 & $Y$ & $18.12 \pm 0.42$ & $188.45 \pm 27.83$ \\
\hline 1.85 & 159668 & 536 & 1.87 & $Z$ & $18.34 \pm 0.42$ & $158.21 \pm 15.76$ \\
\hline 1.87 & 161334 & 1280 & 1.89 & $i$ & $18.55 \pm 0.42$ & $129.04 \pm 2.97$ \\
\hline 1.87 & 161334 & 1280 & 1.89 & $r$ & $18.72 \pm 0.42$ & $110.25 \pm 1.77$ \\
\hline 1.87 & 161335 & 536 & 1.89 & $Y$ & $18.11 \pm 0.42$ & $185.62 \pm 27.80$ \\
\hline 1.87 & 161335 & 536 & 1.89 & $Z$ & $18.34 \pm 0.42$ & $153.69 \pm 17.65$ \\
\hline 1.87 & 161337 & 536 & 1.89 & $H$ & $18.08 \pm 0.43$ & $215.43 \pm 78.45$ \\
\hline 1.87 & 161337 & 536 & 1.89 & $J$ & $18.23 \pm 0.43$ & $169.34 \pm 41.59$ \\
\hline
\end{tabular}


TABLE 6 - Continued

\begin{tabular}{|c|c|c|c|c|c|c|}
\hline$t_{0}(\mathrm{~d})$ & $t_{0}(\mathrm{~s})$ & $t_{e}(\mathrm{~s})$ & $t_{f}(\mathrm{~s})$ & Filter & $\begin{array}{c}m(\mathrm{AB}) \\
\text { (aperture) }\end{array}$ & $\begin{array}{l}\text { Flux }(\mu \mathrm{Jy}) \\
\quad(\mathrm{PSF})\end{array}$ \\
\hline 1.89 & 163694 & 1280 & 1.91 & $i$ & $18.57 \pm 0.42$ & $125.75 \pm 1.90$ \\
\hline 1.89 & 163694 & 1280 & 1.91 & $r$ & $18.72 \pm 0.42$ & $109.57 \pm 1.17$ \\
\hline 1.89 & 163700 & 536 & 1.91 & $Y$ & $18.19 \pm 0.42$ & $178.61 \pm 17.89$ \\
\hline 1.89 & 163700 & 536 & 1.91 & $Z$ & $18.35 \pm 0.42$ & $157.04 \pm 13.47$ \\
\hline 1.89 & 163702 & 536 & 1.91 & $H$ & $18.06 \pm 0.43$ & $219.76 \pm 61.30$ \\
\hline 1.89 & 163702 & 536 & 1.91 & $J$ & $18.34 \pm 0.42$ & $163.73 \pm 28.56$ \\
\hline 1.91 & 165405 & 1280 & 1.93 & $i$ & $18.60 \pm 0.42$ & $124.18 \pm 2.92$ \\
\hline 1.91 & 165405 & 1280 & 1.93 & $r$ & $18.77 \pm 0.42$ & $105.43 \pm 1.43$ \\
\hline 1.91 & 165407 & 536 & 1.93 & $Y$ & $18.20 \pm 0.42$ & $177.57 \pm 18.95$ \\
\hline 1.91 & 165407 & 536 & 1.93 & $Z$ & $18.36 \pm 0.42$ & $153.59 \pm 12.91$ \\
\hline 1.91 & 165408 & 536 & 1.93 & $H$ & $18.14 \pm 0.43$ & $198.83 \pm 46.64$ \\
\hline 1.91 & 165408 & 536 & 1.93 & $J$ & $18.30 \pm 0.42$ & $166.47 \pm 29.66$ \\
\hline 1.93 & 167079 & 1280 & 1.95 & $i$ & $18.60 \pm 0.42$ & $122.24 \pm 2.87$ \\
\hline 1.93 & 167079 & 1280 & 1.95 & $r$ & $18.76 \pm 0.42$ & $104.86 \pm 2.11$ \\
\hline 1.93 & 167081 & 536 & 1.95 & $H$ & $18.06 \pm 0.42$ & $209.93 \pm 56.56$ \\
\hline 1.93 & 167081 & 536 & 1.95 & $J$ & $18.28 \pm 0.42$ & $164.50 \pm 26.93$ \\
\hline 1.93 & 167081 & 536 & 1.95 & $Y$ & $18.23 \pm 0.42$ & $172.74 \pm 18.35$ \\
\hline 1.93 & 167081 & 536 & 1.95 & $Z$ & $18.39 \pm 0.42$ & $152.73 \pm 12.78$ \\
\hline 1.96 & 169250 & 536 & 1.98 & $\bar{H}$ & $18.04 \pm 0.42$ & $209.45 \pm 45.11$ \\
\hline 1.96 & 169250 & 536 & 1.98 & $J$ & $18.34 \pm 0.42$ & $161.81 \pm 61.05$ \\
\hline 1.96 & 169286 & 1280 & 1.98 & $r$ & $18.78 \pm 0.42$ & $104.67 \pm 2.68$ \\
\hline 1.96 & 169286 & 1280 & 1.98 & $i$ & $18.62 \pm 0.42$ & $120.64 \pm 2.56$ \\
\hline 1.96 & 169310 & 469 & 1.98 & $Y$ & $18.29 \pm 0.42$ & $173.81 \pm 21.84$ \\
\hline 1.96 & 169310 & 604 & 1.98 & $Z$ & $18.36 \pm 0.42$ & $153.08 \pm 13.55$ \\
\hline 1.98 & 170985 & 1280 & 2.00 & $i$ & $18.63 \pm 0.42$ & $120.42 \pm 2.36$ \\
\hline 1.98 & 170985 & 1280 & 2.00 & $r$ & $18.80 \pm 0.42$ & $101.31 \pm 2.98$ \\
\hline 1.98 & 170986 & 536 & 2.00 & $H$ & $18.04 \pm 0.43$ & $230.80 \pm 110.74$ \\
\hline 1.98 & 170986 & 536 & 2.00 & $J$ & $18.23 \pm 0.42$ & $164.63 \pm 30.78$ \\
\hline 1.98 & 171108 & 536 & 2.00 & $Y$ & $18.21 \pm 0.42$ & $180.27 \pm 22.90$ \\
\hline 1.98 & 171108 & 536 & 2.00 & $Z$ & $18.45 \pm 0.42$ & $142.52 \pm 16.11$ \\
\hline 2.00 & 172728 & 1280 & 2.02 & $r$ & $18.82 \pm 0.42$ & $98.70 \pm 1.90$ \\
\hline 2.00 & 172728 & 1280 & 2.02 & $i$ & $18.62 \pm 0.42$ & $120.02 \pm 2.87$ \\
\hline 2.00 & 172735 & 536 & 2.02 & $H$ & $18.10 \pm 0.43$ & $219.87 \pm 63.24$ \\
\hline 2.00 & 172735 & 536 & 2.02 & $J$ & $18.37 \pm 0.43$ & $154.87 \pm 31.02$ \\
\hline 2.00 & 173091 & 536 & 2.03 & $Y$ & $18.28 \pm 0.42$ & $159.93 \pm 21.83$ \\
\hline 2.00 & 173091 & 536 & 2.03 & $Z$ & $18.37 \pm 0.42$ & $152.26 \pm 18.02$ \\
\hline 2.02 & 174940 & 1280 & 2.04 & $r$ & $18.83 \pm 0.42$ & $97.36 \pm 2.12$ \\
\hline 2.02 & 174940 & 1280 & 2.04 & $i$ & $18.65 \pm 0.42$ & $117.40 \pm 2.51$ \\
\hline 2.02 & 174946 & 536 & 2.04 & $H$ & $18.23 \pm 0.43$ & $191.66 \pm 95.17$ \\
\hline 2.02 & 174946 & 536 & 2.04 & $J$ & $18.31 \pm 0.43$ & $163.10 \pm 44.53$ \\
\hline 2.03 & 175056 & 536 & 2.05 & $Y$ & $18.32 \pm 0.42$ & $157.62 \pm 25.69$ \\
\hline 2.03 & 175056 & 536 & 2.05 & $Z$ & $18.41 \pm 0.42$ & $146.81 \pm 18.00$ \\
\hline 2.04 & 176669 & 1280 & 2.06 & $i$ & $18.68 \pm 0.42$ & $114.04 \pm 4.09$ \\
\hline 2.04 & 176669 & 1280 & 2.06 & $r$ & $18.85 \pm 0.42$ & $97.26 \pm 2.93$ \\
\hline 2.04 & 176673 & 536 & 2.06 & $H$ & $18.14 \pm 0.43$ & $209.82 \pm 125.97$ \\
\hline 2.04 & 176673 & 536 & 2.06 & $J$ & $18.35 \pm 0.43$ & $161.18 \pm 52.20$ \\
\hline 2.83 & 244162 & 1280 & 2.85 & $i$ & $19.15 \pm 0.42$ & $71.80 \pm 1.38$ \\
\hline 2.83 & 244162 & 1280 & 2.85 & $r$ & $19.28 \pm 0.42$ & $62.18 \pm 0.91$ \\
\hline 2.83 & 244168 & 536 & 2.85 & $H$ & $18.49 \pm 0.43$ & $135.44 \pm 80.88$ \\
\hline 2.83 & 244168 & 536 & 2.85 & $J$ & $18.81 \pm 0.43$ & $94.62 \pm 40.25$ \\
\hline 2.83 & 244171 & 536 & 2.85 & $Y$ & $18.72 \pm 0.43$ & $114.77 \pm 24.01$ \\
\hline 2.83 & 244171 & 536 & 2.85 & $Z$ & $18.84 \pm 0.42$ & $95.64 \pm 8.66$ \\
\hline 2.85 & 245865 & 1280 & 2.86 & $i$ & $19.13 \pm 0.42$ & $72.81 \pm 1.67$ \\
\hline 2.85 & 245865 & 1280 & 2.86 & $r$ & $19.26 \pm 0.42$ & $63.45 \pm 1.38$ \\
\hline 2.85 & 245867 & 469 & 2.86 & $H$ & $18.51 \pm 0.43$ & $117.37 \pm 133.41$ \\
\hline 2.85 & 245867 & 536 & 2.86 & $Y$ & $18.67 \pm 0.43$ & $109.85 \pm 23.93$ \\
\hline 2.85 & 245867 & 536 & 2.86 & $Z$ & $18.92 \pm 0.42$ & $88.60 \pm 9.96$ \\
\hline 2.85 & 245867 & 604 & 2.86 & $J$ & $18.81 \pm 0.43$ & $100.35 \pm 55.57$ \\
\hline 2.87 & 247553 & 1280 & 2.88 & $i$ & $19.14 \pm 0.42$ & $71.86 \pm 1.42$ \\
\hline 2.87 & 247553 & 1280 & 2.88 & $r$ & $19.28 \pm 0.42$ & $63.17 \pm 0.93$ \\
\hline 2.87 & 247555 & 536 & 2.88 & $Y$ & $18.66 \pm 0.43$ & $116.44 \pm 25.16$ \\
\hline 2.87 & 247555 & 536 & 2.88 & $Z$ & $18.91 \pm 0.42$ & $91.74 \pm 8.23$ \\
\hline 2.87 & 247556 & 536 & 2.88 & $\bar{H}$ & $18.56 \pm 0.43$ & $130.53 \pm 76.23$ \\
\hline 2.87 & 247556 & 536 & 2.88 & $J$ & $18.86 \pm 0.43$ & $95.17 \pm 31.71$ \\
\hline 2.89 & 249656 & 536 & 2.91 & $H$ & $18.72 \pm 0.43$ & $116.01 \pm 48.13$ \\
\hline 2.89 & 249656 & 536 & 2.91 & $J$ & $18.89 \pm 0.43$ & $88.72 \pm 22.27$ \\
\hline 2.89 & 249656 & 536 & 2.91 & $Y$ & $18.81 \pm 0.43$ & $94.88 \pm 11.61$ \\
\hline 2.89 & 249656 & 536 & 2.91 & $Z$ & $18.86 \pm 0.42$ & $92.86 \pm 5.93$ \\
\hline 2.89 & 249693 & 1280 & 2.91 & $i$ & $19.16 \pm 0.42$ & $70.79 \pm 1.21$ \\
\hline 2.89 & 249693 & 1280 & 2.91 & $r$ & $19.31 \pm 0.42$ & $61.72 \pm 1.09$ \\
\hline 2.91 & 251349 & 1280 & 2.93 & $i$ & $19.16 \pm 0.42$ & $69.79 \pm 1.31$ \\
\hline 2.91 & 251349 & 1280 & 2.93 & $r$ & $19.32 \pm 0.42$ & $60.07 \pm 1.05$ \\
\hline 2.91 & 251350 & 536 & 2.93 & $Y$ & $18.76 \pm 0.43$ & $99.00 \pm 15.22$ \\
\hline 2.91 & 251350 & 536 & 2.93 & $Z$ & $18.94 \pm 0.42$ & $84.23 \pm 7.06$ \\
\hline 2.91 & 251352 & 536 & 2.93 & $H$ & $18.70 \pm 0.43$ & $118.01 \pm 54.31$ \\
\hline 2.91 & 251352 & 536 & 2.93 & $J$ & $18.88 \pm 0.43$ & $93.52 \pm 23.21$ \\
\hline
\end{tabular}


TABLE 6 - Continued

\begin{tabular}{|c|c|c|c|c|c|c|}
\hline$t_{0}(\mathrm{~d})$ & $t_{0}(\mathrm{~s})$ & $t_{e}(\mathrm{~s})$ & $t_{f}(\mathrm{~s})$ & Filter & $\begin{array}{c}m(\mathrm{AB}) \\
\text { (aperture) }\end{array}$ & $\begin{array}{c}\text { Flux } \\
(\mu \mathrm{PSF})\end{array}$ \\
\hline 2.93 & 253022 & 1280 & 2.95 & $i$ & $19.18 \pm 0.42$ & $69.02 \pm 1.33$ \\
\hline 2.93 & 253022 & 1280 & 2.95 & $r$ & $19.33 \pm 0.42$ & $59.30 \pm 1.37$ \\
\hline 2.93 & 253024 & 536 & 2.95 & $Y$ & $18.77 \pm 0.43$ & $97.99 \pm 25.24$ \\
\hline 2.93 & 253024 & 536 & 2.95 & $Z$ & $18.94 \pm 0.42$ & $85.20 \pm 7.17$ \\
\hline 2.93 & 253024 & 536 & 2.95 & $H$ & $18.60 \pm 0.43$ & $130.78 \pm 58.04$ \\
\hline 2.93 & 253024 & 536 & 2.95 & $J$ & $18.86 \pm 0.43$ & $96.18 \pm 22.58$ \\
\hline 2.95 & 255197 & 536 & 2.97 & $Y$ & $18.70 \pm 0.43$ & $104.72 \pm 17.90$ \\
\hline 2.95 & 255197 & 536 & 2.97 & $Z$ & $18.89 \pm 0.42$ & $88.48 \pm 7.17$ \\
\hline 2.95 & 255199 & 536 & 2.97 & $H$ & $18.70 \pm 0.43$ & $120.87 \pm 62.41$ \\
\hline 2.95 & 255199 & 536 & 2.97 & $J$ & $18.85 \pm 0.43$ & $98.59 \pm 21.59$ \\
\hline 2.95 & 255236 & 1280 & 2.97 & $r$ & $19.36 \pm 0.42$ & $58.44 \pm 1.39$ \\
\hline 2.95 & 255236 & 1280 & 2.97 & $i$ & $19.18 \pm 0.42$ & $68.25 \pm 1.39$ \\
\hline 2.97 & 256928 & 1280 & 2.99 & $r$ & $19.38 \pm 0.42$ & $57.44 \pm 1.10$ \\
\hline 2.97 & 256930 & 1280 & 2.99 & $i$ & $19.19 \pm 0.42$ & $67.23 \pm 1.40$ \\
\hline 2.97 & 256934 & 536 & 2.99 & $H$ & $18.66 \pm 0.43$ & $137.34 \pm 72.34$ \\
\hline 2.97 & 256934 & 536 & 2.99 & $J$ & $18.92 \pm 0.43$ & $91.18 \pm 22.10$ \\
\hline 2.97 & 256936 & 536 & 2.99 & $Y$ & $18.77 \pm 0.43$ & $98.98 \pm 17.71$ \\
\hline 2.97 & 256936 & 536 & 2.99 & $Z$ & $19.03 \pm 0.42$ & $82.69 \pm 8.79$ \\
\hline 2.99 & 258662 & 1280 & 3.01 & $i$ & $19.17 \pm 0.42$ & $69.61 \pm 1.43$ \\
\hline 2.99 & 258662 & 1280 & 3.01 & $r$ & $19.37 \pm 0.42$ & $57.93 \pm 1.33$ \\
\hline 2.99 & 258663 & 536 & 3.01 & $Y$ & $18.74 \pm 0.43$ & $102.65 \pm 22.76$ \\
\hline 2.99 & 258663 & 536 & 3.01 & $Z$ & $18.96 \pm 0.42$ & $80.52 \pm 9.23$ \\
\hline 2.99 & 258664 & 536 & 3.01 & $H$ & $18.68 \pm 0.43$ & $112.91 \pm 91.56$ \\
\hline 2.99 & 258664 & 536 & 3.01 & $J$ & $18.80 \pm 0.43$ & $93.21 \pm 27.34$ \\
\hline 3.02 & 260909 & 536 & 3.04 & $Y$ & $18.72 \pm 0.43$ & $101.24 \pm 20.76$ \\
\hline 3.02 & 260909 & 536 & 3.04 & $Z$ & $18.93 \pm 0.42$ & $81.85 \pm 8.58$ \\
\hline 3.02 & 260910 & 536 & 3.04 & $H$ & $18.67 \pm 0.43$ & $123.48 \pm 84.77$ \\
\hline 3.02 & 260910 & 536 & 3.04 & $J$ & $18.83 \pm 0.43$ & $96.51 \pm 26.43$ \\
\hline 3.02 & 260945 & 1280 & 3.04 & $r$ & $19.40 \pm 0.42$ & $54.45 \pm 1.09$ \\
\hline 3.02 & 260945 & 1280 & 3.04 & $i$ & $19.19 \pm 0.42$ & $69.98 \pm 1.71$ \\
\hline 3.04 & 262654 & 1280 & 3.06 & $i$ & $19.23 \pm 0.42$ & $65.45 \pm 1.41$ \\
\hline 3.04 & 262654 & 1280 & 3.06 & $r$ & $19.36 \pm 0.42$ & $58.12 \pm 1.37$ \\
\hline 3.04 & 262658 & 536 & 3.06 & $H$ & $18.57 \pm 0.43$ & $123.33 \pm 90.72$ \\
\hline 3.04 & 262658 & 536 & 3.06 & $J$ & $18.93 \pm 0.43$ & $89.83 \pm 30.08$ \\
\hline 3.04 & 262659 & 536 & 3.06 & $Y$ & $18.84 \pm 0.43$ & $94.95 \pm 20.75$ \\
\hline 3.04 & 262659 & 536 & 3.06 & $Z$ & $19.01 \pm 0.43$ & $81.33 \pm 9.88$ \\
\hline 3.82 & 330101 & 1280 & 3.84 & $r$ & $19.70 \pm 0.42$ & $40.20 \pm 1.12$ \\
\hline 3.82 & 330102 & 1280 & 3.84 & $i$ & $19.53 \pm 0.42$ & $47.78 \pm 1.20$ \\
\hline 3.84 & 331631 & 1280 & 3.86 & $r$ & $19.65 \pm 0.42$ & $41.40 \pm 0.96$ \\
\hline 3.84 & 331631 & 1280 & 3.86 & $i$ & $19.50 \pm 0.42$ & $48.77 \pm 1.08$ \\
\hline 3.86 & 333148 & 1280 & 3.87 & $r$ & $19.63 \pm 0.42$ & $41.75 \pm 0.89$ \\
\hline 3.86 & 333148 & 1280 & 3.87 & $i$ & $19.54 \pm 0.42$ & $47.57 \pm 0.92$ \\
\hline 3.88 & 335229 & 1280 & 3.90 & $r$ & $19.69 \pm 0.42$ & $40.12 \pm 0.84$ \\
\hline 3.88 & 335229 & 1280 & 3.90 & $i$ & $19.54 \pm 0.42$ & $47.09 \pm 1.01$ \\
\hline 3.90 & 336754 & 1280 & 3.92 & $r$ & $19.69 \pm 0.42$ & $39.64 \pm 1.01$ \\
\hline 3.90 & 336754 & 1280 & 3.92 & $i$ & $19.54 \pm 0.42$ & $45.71 \pm 0.98$ \\
\hline 3.92 & 338278 & 1280 & 3.93 & $r$ & $19.72 \pm 0.42$ & $40.58 \pm 0.89$ \\
\hline 3.92 & 338279 & 1280 & 3.93 & $i$ & $19.54 \pm 0.42$ & $46.84 \pm 1.07$ \\
\hline 3.94 & 340206 & 1280 & 3.95 & $r$ & $19.70 \pm 0.42$ & $39.45 \pm 1.02$ \\
\hline 3.94 & 340206 & 1280 & 3.95 & $i$ & $19.54 \pm 0.42$ & $46.35 \pm 1.00$ \\
\hline 3.96 & 341738 & 1280 & 3.97 & $r$ & $19.74 \pm 0.42$ & $38.82 \pm 0.82$ \\
\hline 3.96 & 341738 & 1280 & 3.97 & $i$ & $19.56 \pm 0.42$ & $46.04 \pm 0.90$ \\
\hline 3.97 & 343270 & 1280 & 3.99 & $r$ & $19.70 \pm 0.42$ & $39.81 \pm 0.91$ \\
\hline 3.97 & 343271 & 1280 & 3.99 & $i$ & $19.53 \pm 0.42$ & $47.72 \pm 1.11$ \\
\hline 4.00 & 345316 & 1280 & 4.01 & $r$ & $19.73 \pm 0.42$ & $38.54 \pm 0.86$ \\
\hline 4.00 & 345316 & 1280 & 4.01 & $i$ & $19.57 \pm 0.42$ & $45.09 \pm 0.87$ \\
\hline 4.01 & 346882 & 1280 & 4.03 & $r$ & $19.72 \pm 0.42$ & $39.89 \pm 0.94$ \\
\hline 4.01 & 346882 & 1280 & 4.03 & $i$ & $19.56 \pm 0.42$ & $47.26 \pm 1.00$ \\
\hline 4.03 & 348470 & 1280 & 4.05 & $r$ & $19.75 \pm 0.42$ & $38.51 \pm 1.01$ \\
\hline 4.03 & 348471 & 1280 & 4.05 & $i$ & $19.62 \pm 0.42$ & $44.24 \pm 1.18$ \\
\hline 5.03 & 434444 & 1280 & 5.05 & $g$ & $20.35 \pm 0.42$ & $21.96 \pm 1.09$ \\
\hline 5.03 & 434467 & 2320 & 5.08 & $r$ & $20.06 \pm 0.42$ & $25.27 \pm 1.34$ \\
\hline 5.03 & 434468 & 3839 & 5.08 & $i$ & $19.84 \pm 0.42$ & $33.42 \pm 1.14$ \\
\hline 5.94 & 513072 & 5119 & 6.11 & $r$ & $20.16 \pm 0.42$ & $23.64 \pm 0.55$ \\
\hline 5.95 & 513912 & 10240 & 6.14 & $i$ & $20.02 \pm 0.42$ & $27.34 \pm 0.56$ \\
\hline 5.95 & 513920 & 4228 & 6.14 & $Y$ & $19.68 \pm 0.42$ & $36.96 \pm 2.04$ \\
\hline 5.95 & 513920 & 4295 & 6.14 & $Z$ & $19.82 \pm 0.42$ & $31.53 \pm 1.24$ \\
\hline 5.95 & 513921 & 4295 & 6.14 & $H$ & $19.45 \pm 0.43$ & $42.74 \pm 7.19$ \\
\hline 5.95 & 513921 & 4295 & 6.14 & $J$ & $19.66 \pm 0.42$ & $41.53 \pm 2.91$ \\
\hline 5.97 & 515483 & 5119 & 6.12 & $g$ & $20.54 \pm 0.42$ & $17.98 \pm 0.50$ \\
\hline 6.93 & 598530 & 12400 & 7.13 & $i$ & $20.16 \pm 0.42$ & $22.37 \pm 0.55$ \\
\hline 6.93 & 598765 & 11759 & 7.13 & $g$ & $20.67 \pm 0.42$ & $15.46 \pm 0.49$ \\
\hline 6.93 & 598772 & 4832 & 7.13 & $\dot{Y}$ & $19.87 \pm 0.43$ & $30.39 \pm 2.19$ \\
\hline 6.93 & 598772 & 5033 & 7.13 & $Z$ & $19.90 \pm 0.42$ & $28.59 \pm 1.23$ \\
\hline 6.93 & 598776 & 4966 & 7.13 & $H$ & $19.67 \pm 0.43$ & $32.84 \pm 7.91$ \\
\hline 6.93 & 598776 & 5033 & 7.13 & $J$ & $19.82 \pm 0.43$ & $35.80 \pm 5.87$ \\
\hline
\end{tabular}


TABLE 6 - Continued

\begin{tabular}{|c|c|c|c|c|c|c|}
\hline$t_{0}(\mathrm{~d})$ & $t_{0}(\mathrm{~s})$ & $t_{e}(\mathrm{~s})$ & $t_{f}(\mathrm{~s})$ & Filter & $\begin{array}{c}m(\mathrm{AB}) \\
\text { (aperture) }\end{array}$ & $\begin{array}{l}\text { Flux }(\mu \mathrm{Jy}) \\
(\mathrm{PSF})\end{array}$ \\
\hline 7.94 & 686416 & 9040 & 8.14 & $g$ & $20.82 \pm 0.42$ & $12.77 \pm 0.51$ \\
\hline 7.94 & 686416 & 9679 & 8.14 & $i$ & $20.31 \pm 0.42$ & $18.42 \pm 0.56$ \\
\hline 7.95 & 686463 & 3557 & 8.14 & $Z$ & $20.07 \pm 0.43$ & $22.98 \pm 1.38$ \\
\hline 7.95 & 686463 & 3557 & 8.14 & $J$ & $19.80 \pm 0.43$ & $33.50 \pm 4.67$ \\
\hline 7.95 & 686463 & 4093 & 8.14 & $Y$ & $19.87 \pm 0.43$ & $28.88 \pm 2.74$ \\
\hline 7.95 & 686463 & 4698 & 8.14 & $H$ & $19.80 \pm 0.43$ & $51.25 \pm 11.57$ \\
\hline 9.96 & 860894 & 5999 & 10.08 & $g$ & $21.04 \pm 0.42$ & $9.46 \pm 0.49$ \\
\hline 9.96 & 860894 & 5999 & 10.08 & $i$ & $20.46 \pm 0.42$ & $14.73 \pm 0.57$ \\
\hline 9.96 & 860902 & 2483 & 10.08 & $J$ & $20.22 \pm 0.43$ & $19.00 \pm 5.64$ \\
\hline 9.96 & 860902 & 2550 & 10.08 & $H$ & $20.19 \pm 0.44$ & $-1.10 \pm 15.50$ \\
\hline 9.96 & 860904 & 2483 & 10.08 & $Z$ & $20.18 \pm 0.43$ & $18.27 \pm 1.70$ \\
\hline 9.96 & 860904 & 2550 & 10.08 & $\bar{Y}$ & $20.18 \pm 0.43$ & $20.30 \pm 3.31$ \\
\hline 10.91 & 943033 & 1120 & 10.93 & $r$ & $20.64 \pm 0.43$ & $11.51 \pm 1.26$ \\
\hline 10.93 & 943968 & 3892 & 11.15 & $Z$ & $20.35 \pm 0.43$ & $13.97 \pm 1.59$ \\
\hline 10.93 & 943968 & 3959 & 11.15 & $Y$ & $20.27 \pm 0.43$ & $18.42 \pm 3.49$ \\
\hline 10.93 & 944062 & 9840 & 11.15 & $i$ & $20.54 \pm 0.42$ & $13.32 \pm 0.58$ \\
\hline 10.93 & 944113 & 2480 & 11.15 & $g$ & $21.17 \pm 0.43$ & $8.07 \pm 0.83$ \\
\hline 10.93 & 944130 & 4093 & 11.15 & $J$ & $20.21 \pm 0.44$ & $24.43 \pm 9.14$ \\
\hline 11.93 & 1030399 & 4832 & 12.13 & $Y$ & $20.38 \pm 0.43$ & $15.22 \pm 1.38$ \\
\hline 11.93 & 1030399 & 4832 & 12.13 & $Z$ & $20.40 \pm 0.42$ & $13.84 \pm 0.89$ \\
\hline 11.93 & 1030400 & 4832 & 12.13 & $H$ & $20.09 \pm 0.43$ & $18.47 \pm 4.85$ \\
\hline 11.93 & 1030400 & 4832 & 12.13 & $J$ & $20.43 \pm 0.43$ & $12.27 \pm 2.19$ \\
\hline 11.93 & 1030435 & 6399 & 12.13 & $r$ & $20.68 \pm 0.42$ & $10.92 \pm 0.52$ \\
\hline 11.93 & 1030435 & 5119 & 12.09 & $g$ & $21.12 \pm 0.42$ & $8.26 \pm 0.49$ \\
\hline 11.93 & 1030435 & 11519 & 12.13 & $i$ & $20.58 \pm 0.42$ & $12.27 \pm 0.49$ \\
\hline 12.91 & 1115381 & 5119 & 13.09 & $r$ & $20.71 \pm 0.42$ & $10.44 \pm 0.54$ \\
\hline 12.91 & 1115381 & 10240 & 13.09 & $i$ & $20.60 \pm 0.42$ & $11.86 \pm 0.50$ \\
\hline 12.91 & 1115386 & 4295 & 13.09 & $Y$ & $20.33 \pm 0.43$ & $16.63 \pm 1.77$ \\
\hline 12.91 & 1115386 & 4295 & 13.09 & $Z$ & $20.39 \pm 0.43$ & $13.92 \pm 1.02$ \\
\hline 12.91 & 1115413 & 5119 & 13.05 & $g$ & $21.17 \pm 0.42$ & $7.90 \pm 0.49$ \\
\hline 12.91 & 1115428 & 4295 & 13.09 & $\vec{H}$ & $20.34 \pm 0.44$ & $2.34 \pm 6.49$ \\
\hline 12.91 & 1115428 & 4295 & 13.09 & $J$ & $20.34 \pm 0.43$ & $16.98 \pm 3.20$ \\
\hline 13.91 & 1201686 & 5119 & 14.04 & $g$ & $21.24 \pm 0.42$ & $7.36 \pm 0.48$ \\
\hline 13.91 & 1201710 & 5119 & 14.08 & $r$ & $20.76 \pm 0.42$ & $9.59 \pm 0.54$ \\
\hline 13.91 & 1201710 & 10160 & 14.08 & $i$ & $20.67 \pm 0.42$ & $10.39 \pm 0.50$ \\
\hline 13.91 & 1201715 & 4228 & 14.08 & $Y$ & $20.40 \pm 0.43$ & $14.09 \pm 1.74$ \\
\hline 13.91 & 1201715 & 4295 & 14.08 & $Z$ & $20.52 \pm 0.43$ & $11.19 \pm 0.97$ \\
\hline 13.91 & 1201716 & 4295 & 14.08 & $H$ & $20.33 \pm 0.44$ & $0.08 \pm 6.63$ \\
\hline 13.91 & 1201716 & 4295 & 14.08 & $J$ & $20.36 \pm 0.43$ & $18.58 \pm 2.92$ \\
\hline 14.90 & 1287710 & 5119 & 15.05 & $r$ & $20.78 \pm 0.42$ & $9.55 \pm 0.53$ \\
\hline 14.91 & 1288530 & 10240 & 15.08 & $i$ & $20.72 \pm 0.42$ & $10.15 \pm 0.49$ \\
\hline 14.91 & 1288579 & 4295 & 15.08 & $H$ & $20.26 \pm 0.43$ & $16.57 \pm 4.77$ \\
\hline 14.91 & 1288579 & 4295 & 15.08 & $J$ & $20.53 \pm 0.43$ & $15.81 \pm 2.29$ \\
\hline 14.91 & 1288581 & 4295 & 15.08 & $Y$ & $20.41 \pm 0.43$ & $13.66 \pm 1.38$ \\
\hline 14.91 & 1288581 & 4295 & 15.08 & $Z$ & $20.49 \pm 0.43$ & $11.03 \pm 0.89$ \\
\hline 14.92 & 1289370 & 5119 & 15.07 & $g$ & $21.26 \pm 0.42$ & $6.95 \pm 0.49$ \\
\hline 15.89 & 1373149 & 5119 & 16.03 & $r$ & $20.83 \pm 0.42$ & $8.62 \pm 0.55$ \\
\hline 15.90 & 1373982 & 10240 & 16.06 & $i$ & $20.75 \pm 0.42$ & $9.25 \pm 0.50$ \\
\hline 15.90 & 1374029 & 4295 & 16.06 & $Y$ & $20.52 \pm 0.43$ & $11.67 \pm 1.67$ \\
\hline 15.90 & 1374029 & 4295 & 16.06 & $Z$ & $20.54 \pm 0.43$ & $10.73 \pm 1.03$ \\
\hline 15.90 & 1374030 & 4295 & 16.06 & $H$ & $20.36 \pm 0.44$ & $5.63 \pm 5.26$ \\
\hline 15.90 & 1374030 & 4295 & 16.06 & $J$ & $20.60 \pm 0.43$ & $8.97 \pm 2.75$ \\
\hline 15.91 & 1374818 & 5119 & 16.05 & $g$ & $21.29 \pm 0.42$ & $6.83 \pm 0.50$ \\
\hline 16.91 & 1460973 & 4480 & 17.04 & $r$ & $20.81 \pm 0.42$ & $9.13 \pm 0.57$ \\
\hline 16.91 & 1460973 & 8319 & 17.04 & $i$ & $20.75 \pm 0.42$ & $9.38 \pm 0.52$ \\
\hline 16.91 & 1460979 & 3489 & 17.04 & $Y$ & $20.55 \pm 0.43$ & $10.18 \pm 2.47$ \\
\hline 16.91 & 1460979 & 3489 & 17.04 & $Z$ & $20.60 \pm 0.43$ & $10.59 \pm 1.12$ \\
\hline 16.91 & 1460981 & 3489 & 17.04 & $J$ & $20.55 \pm 0.44$ & $8.21 \pm 3.78$ \\
\hline 16.91 & 1461359 & 3839 & 17.02 & $g$ & $21.33 \pm 0.42$ & $6.47 \pm 0.52$ \\
\hline 17.91 & 1547770 & 3839 & 18.06 & $r$ & $20.87 \pm 0.42$ & $8.51 \pm 0.61$ \\
\hline 17.93 & 1548872 & 7680 & 18.09 & $i$ & $20.75 \pm 0.42$ & $9.16 \pm 0.55$ \\
\hline 17.93 & 1548882 & 3221 & 18.09 & $Y$ & $20.59 \pm 0.43$ & $8.06 \pm 2.81$ \\
\hline 17.93 & 1548882 & 3221 & 18.09 & $Z$ & $20.61 \pm 0.43$ & $9.09 \pm 1.38$ \\
\hline 17.94 & 1549668 & 3839 & 18.08 & $g$ & $21.39 \pm 0.42$ & $5.89 \pm 0.55$ \\
\hline 18.92 & 1634492 & 6399 & 19.11 & $r$ & $20.91 \pm 0.42$ & $7.38 \pm 0.54$ \\
\hline 18.92 & 1634492 & 11359 & 19.11 & $i$ & $20.79 \pm 0.42$ & $8.55 \pm 0.50$ \\
\hline 18.92 & 1634525 & 4959 & 19.07 & $g$ & $21.38 \pm 0.42$ & $5.56 \pm 0.50$ \\
\hline 18.92 & 1634538 & 4765 & 19.10 & $J$ & $20.48 \pm 0.43$ & $13.30 \pm 2.52$ \\
\hline 18.92 & 1634542 & 4765 & 19.10 & $Y$ & $20.57 \pm 0.43$ & $11.37 \pm 1.61$ \\
\hline 18.92 & 1634542 & 4765 & 19.10 & $Z$ & $20.59 \pm 0.43$ & $10.29 \pm 0.93$ \\
\hline 19.91 & 1720548 & 6079 & 20.09 & $r$ & $20.91 \pm 0.42$ & $7.22 \pm 0.57$ \\
\hline 19.91 & 1720548 & 11120 & 20.09 & $i$ & $20.82 \pm 0.42$ & $8.25 \pm 0.52$ \\
\hline 19.91 & 1720583 & 5119 & 20.05 & $g$ & $21.43 \pm 0.42$ & $5.28 \pm 0.51$ \\
\hline 19.91 & 1720594 & 4563 & 20.09 & $J$ & $20.59 \pm 0.43$ & $7.48 \pm 2.76$ \\
\hline 19.91 & 1720597 & 4429 & 20.09 & $Y$ & $20.57 \pm 0.43$ & $10.06 \pm 2.14$ \\
\hline 19.91 & 1720597 & 4496 & 20.09 & $Z$ & $20.70 \pm 0.43$ & $9.23 \pm 1.08$ \\
\hline
\end{tabular}


TABLE 6 - Continued

\begin{tabular}{|c|c|c|c|c|c|c|}
\hline$t_{0}(\mathrm{~d})$ & $t_{0}(\mathrm{~s})$ & $t_{e}(\mathrm{~s})$ & $t_{f}(\mathrm{~s})$ & Filter & $\begin{array}{c}m(\mathrm{AB}) \\
\text { (aperture) }\end{array}$ & $\begin{array}{l}\text { Flux }(\mu \mathrm{Jy}) \\
(\mathrm{PSF})\end{array}$ \\
\hline 20.91 & 1807055 & 6399 & 21.10 & $r$ & $20.91 \pm 0.42$ & $7.10 \pm 0.61$ \\
\hline 20.91 & 1807055 & 11439 & 21.10 & $i$ & $20.79 \pm 0.42$ & $8.25 \pm 0.56$ \\
\hline 20.92 & 1807079 & 5039 & 21.06 & $g$ & $21.52 \pm 0.43$ & $4.36 \pm 0.57$ \\
\hline 20.92 & 1807103 & 4765 & 21.10 & $J^{3}$ & $20.78 \pm 0.44$ & $7.57 \pm 3.74$ \\
\hline 20.92 & 1807104 & 4765 & 21.10 & $Z$ & $20.58 \pm 0.43$ & $10.89 \pm 1.36$ \\
\hline 20.92 & 1807104 & 4832 & 21.10 & $Y$ & $20.60 \pm 0.43$ & $9.30 \pm 2.26$ \\
\hline 21.92 & 1893510 & 6399 & 22.10 & $r$ & $20.96 \pm 0.42$ & $6.56 \pm 0.62$ \\
\hline 21.92 & 1893511 & 11519 & 22.10 & $i$ & $20.84 \pm 0.42$ & $7.54 \pm 0.57$ \\
\hline 21.92 & 1893520 & 5119 & 22.06 & $g$ & $21.51 \pm 0.43$ & $4.19 \pm 0.57$ \\
\hline 21.92 & 1893559 & 4832 & 22.09 & $J$ & $21.04 \pm 0.45$ & $5.14 \pm 3.19$ \\
\hline 21.92 & 1893561 & 4832 & 22.09 & $Z$ & $20.59 \pm 0.43$ & $10.00 \pm 1.58$ \\
\hline 21.92 & 1893561 & 4832 & 22.09 & $Y$ & $20.64 \pm 0.43$ & $7.93 \pm 2.18$ \\
\hline 22.91 & 1979266 & 5119 & 23.04 & $r$ & $20.94 \pm 0.42$ & $6.60 \pm 0.59$ \\
\hline 22.92 & 1980062 & 10240 & 23.07 & $i$ & $20.87 \pm 0.42$ & $7.27 \pm 0.51$ \\
\hline 22.93 & 1980833 & 5119 & 23.06 & $g$ & $21.53 \pm 0.43$ & $5.10 \pm 0.53$ \\
\hline 23.91 & 2065542 & 4959 & 24.04 & $g$ & $21.46 \pm 0.43$ & $4.78 \pm 0.59$ \\
\hline 23.91 & 2065553 & 6399 & 24.08 & $r$ & $20.91 \pm 0.42$ & $6.21 \pm 0.66$ \\
\hline 23.91 & 2065553 & 11519 & 24.08 & $i$ & $20.86 \pm 0.42$ & $7.29 \pm 0.54$ \\
\hline 24.99 & 2159267 & 1200 & 25.01 & $r$ & $21.24 \pm 0.44$ & $2.72 \pm 1.41$ \\
\hline 24.99 & 2159267 & 1200 & 25.01 & $i$ & $20.92 \pm 0.43$ & $6.83 \pm 1.54$ \\
\hline 25.90 & 2237953 & 4879 & 26.05 & $r$ & $21.10 \pm 0.43$ & $5.15 \pm 0.70$ \\
\hline 25.91 & 2238775 & 10000 & 26.08 & $i$ & $20.91 \pm 0.42$ & $6.82 \pm 0.58$ \\
\hline 25.91 & 2238821 & 4161 & 26.08 & $Y$ & $20.85 \pm 0.44$ & $7.22 \pm 2.92$ \\
\hline 25.91 & 2238821 & 4228 & 26.08 & $Z$ & $20.78 \pm 0.43$ & $-0.23 \pm 1.43$ \\
\hline 25.92 & 2239618 & 5039 & 26.07 & $g$ & $21.61 \pm 0.43$ & $3.91 \pm 0.67$ \\
\hline 26.91 & 2325407 & 3839 & 27.01 & $g$ & $21.69 \pm 0.43$ & $0.00 \pm 0.44$ \\
\hline 26.92 & 2325639 & 5119 & 27.06 & $r$ & $21.07 \pm 0.43$ & $4.87 \pm 0.67$ \\
\hline 26.92 & 2325639 & 8960 & 27.06 & $i$ & $20.86 \pm 0.42$ & $7.12 \pm 0.57$ \\
\hline 26.92 & 2325685 & 3758 & 27.06 & $J$ & $20.56 \pm 0.44$ & $11.38 \pm 4.12$ \\
\hline 26.92 & 2325688 & 3758 & 27.06 & $Z$ & $20.72 \pm 0.43$ & $6.95 \pm 1.48$ \\
\hline 26.92 & 2325688 & 3758 & 27.06 & $Y$ & $20.84 \pm 0.44$ & $3.92 \pm 2.48$ \\
\hline 27.90 & 2410506 & 5119 & 28.04 & $r$ & $21.09 \pm 0.43$ & $5.85 \pm 0.74$ \\
\hline 27.91 & 2411320 & 10240 & 28.07 & $i$ & $20.96 \pm 0.42$ & $6.13 \pm 0.58$ \\
\hline 27.91 & 2411368 & 4295 & 28.07 & $Z$ & $20.84 \pm 0.43$ & $0.76 \pm 1.29$ \\
\hline 27.91 & 2411368 & 4295 & 28.07 & $Y$ & $20.86 \pm 0.44$ & $4.35 \pm 2.39$ \\
\hline 27.92 & 2412156 & 5119 & 28.06 & $g$ & $21.64 \pm 0.43$ & $3.39 \pm 0.73$ \\
\hline 28.90 & 2496872 & 4959 & 29.04 & $r$ & $21.13 \pm 0.43$ & $4.23 \pm 0.63$ \\
\hline 28.91 & 2497696 & 10080 & 29.07 & $i$ & $20.91 \pm 0.42$ & $6.33 \pm 0.55$ \\
\hline 28.91 & 2497747 & 4228 & 29.07 & $Y$ & $20.70 \pm 0.43$ & $4.82 \pm 1.99$ \\
\hline 28.91 & 2497747 & 4228 & 29.07 & $Z$ & $20.79 \pm 0.43$ & $-0.11 \pm 1.18$ \\
\hline 28.92 & 2498536 & 5119 & 29.06 & $g$ & $21.75 \pm 0.43$ & $3.35 \pm 0.63$ \\
\hline 29.92 & 2584960 & 3520 & 30.02 & $r$ & $21.15 \pm 0.43$ & $4.92 \pm 0.66$ \\
\hline 29.93 & 2586106 & 7119 & 30.06 & $i$ & $21.00 \pm 0.42$ & $5.39 \pm 0.59$ \\
\hline 29.93 & 2586153 & 3020 & 30.06 & $Z$ & $20.81 \pm 0.43$ & $-1.40 \pm 1.53$ \\
\hline 29.94 & 2586921 & 3440 & 30.05 & $g$ & $21.78 \pm 0.43$ & $2.83 \pm 0.63$ \\
\hline 30.91 & 2670326 & 3199 & 31.00 & $g$ & $21.90 \pm 0.43$ & $2.35 \pm 0.63$ \\
\hline 30.91 & 2670536 & 4879 & 31.05 & $r$ & $21.30 \pm 0.43$ & $2.88 \pm 0.63$ \\
\hline 30.91 & 2670536 & 8080 & 31.05 & $i$ & $20.97 \pm 0.42$ & $5.52 \pm 0.57$ \\
\hline 30.91 & 2670583 & 3355 & 31.05 & $Y$ & $20.66 \pm 0.43$ & $3.27 \pm 2.66$ \\
\hline 30.91 & 2670583 & 3422 & 31.05 & $Z$ & $20.66 \pm 0.43$ & $3.83 \pm 1.43$ \\
\hline 31.90 & 2756306 & 3680 & 32.00 & $g$ & $21.82 \pm 0.43$ & $2.53 \pm 0.53$ \\
\hline 31.90 & 2756574 & 5119 & 32.05 & $r$ & $21.22 \pm 0.42$ & $2.92 \pm 0.57$ \\
\hline 31.90 & 2756574 & 8800 & 32.05 & $i$ & $21.00 \pm 0.42$ & $5.30 \pm 0.54$ \\
\hline 31.91 & 2756622 & 3691 & 32.05 & $Y$ & $20.73 \pm 0.43$ & $6.41 \pm 2.60$ \\
\hline 31.91 & 2756622 & 3691 & 32.05 & $Z$ & $20.81 \pm 0.43$ & $3.59 \pm 1.38$ \\
\hline 32.90 & 2842792 & 3440 & 33.00 & $g$ & $21.83 \pm 0.43$ & $2.21 \pm 0.54$ \\
\hline 32.91 & 2843068 & 4879 & 33.05 & $r$ & $21.22 \pm 0.43$ & $2.78 \pm 0.60$ \\
\hline 32.91 & 2843068 & 8319 & 33.05 & $i$ & $21.01 \pm 0.42$ & $5.33 \pm 0.57$ \\
\hline 32.91 & 2843117 & 3557 & 33.05 & $Z$ & $20.74 \pm 0.43$ & $2.95 \pm 1.64$ \\
\hline 33.90 & 2929382 & 3839 & 34.01 & $g$ & $21.86 \pm 0.43$ & $2.34 \pm 0.48$ \\
\hline 33.91 & 2929674 & 4800 & 34.05 & $r$ & $21.32 \pm 0.43$ & $2.93 \pm 0.93$ \\
\hline 33.91 & 2929674 & 8960 & 34.05 & $i$ & $21.02 \pm 0.42$ & $4.89 \pm 0.49$ \\
\hline 34.89 & 3014902 & 3839 & 35.00 & $g$ & $21.91 \pm 0.43$ & $2.23 \pm 0.48$ \\
\hline 34.90 & 3015214 & 8960 & 35.05 & $i$ & $21.06 \pm 0.42$ & $4.78 \pm 0.50$ \\
\hline 35.90 & 3101699 & 3839 & 36.00 & $g$ & $21.95 \pm 0.43$ & $1.58 \pm 0.49$ \\
\hline 35.90 & 3101980 & 8960 & 36.05 & $i$ & $21.06 \pm 0.42$ & $4.31 \pm 0.49$ \\
\hline 35.90 & 3102033 & 2160 & 36.05 & $r$ & $21.23 \pm 0.44$ & $3.30 \pm 1.50$ \\
\hline 36.89 & 3187381 & 3520 & 37.00 & $r$ & $21.71 \pm 0.43$ & $-0.70 \pm 0.77$ \\
\hline 36.90 & 3188503 & 8080 & 37.04 & $i$ & $21.08 \pm 0.42$ & $4.42 \pm 0.52$ \\
\hline 38.88 & 3359533 & 3839 & 38.98 & $r$ & $21.33 \pm 0.43$ & $2.55 \pm 0.58$ \\
\hline 38.89 & 3360354 & 7680 & 39.01 & $i$ & $21.13 \pm 0.42$ & $3.64 \pm 0.53$ \\
\hline 38.89 & 3360403 & 3221 & 39.01 & $Z$ & $20.83 \pm 0.43$ & $1.30 \pm 45.53$ \\
\hline 38.90 & 3361179 & 3839 & 39.00 & $g$ & $22.07 \pm 0.43$ & $1.24 \pm 0.51$ \\
\hline 39.90 & 3446958 & 7680 & 40.01 & $r$ & $21.34 \pm 0.42$ & $2.29 \pm 0.51$ \\
\hline 39.90 & 3446958 & 7680 & 40.01 & $i$ & $21.14 \pm 0.42$ & $3.80 \pm 0.49$ \\
\hline 39.90 & 3447005 & 6442 & 40.01 & $H$ & $20.73 \pm 0.43$ & $1.81 \pm 2.42$ \\
\hline
\end{tabular}


TABLE 6 - Continued

\begin{tabular}{|c|c|c|c|c|c|c|}
\hline$t_{0}(\mathrm{~d})$ & $t_{0}(\mathrm{~s})$ & $t_{e}(\mathrm{~s})$ & $t_{f}(\mathrm{~s})$ & Filter & $\begin{array}{c}m(\mathrm{AB}) \\
\text { (aperture) }\end{array}$ & $\begin{array}{l}\text { Flux }(\mu \mathrm{Jy}) \\
\quad(\mathrm{PSF})\end{array}$ \\
\hline 39.90 & 3447006 & 6442 & 40.01 & $Y$ & $20.93 \pm 0.43$ & $2.26 \pm 0.94$ \\
\hline 42.92 & 3708410 & 2640 & 42.99 & $i$ & $21.23 \pm 0.43$ & $4.70 \pm 8.51$ \\
\hline 42.92 & 3708417 & 2214 & 42.99 & $Z$ & $21.25 \pm 0.44$ & $0.28 \pm 1.40$ \\
\hline 44.88 & 3877978 & 6399 & 44.98 & $g$ & $21.98 \pm 0.43$ & $1.37 \pm 0.48$ \\
\hline 44.88 & 3877978 & 6399 & 44.98 & $i$ & $21.18 \pm 0.42$ & $3.12 \pm 0.51$ \\
\hline 46.89 & 4051373 & 6560 & 47.00 & $r$ & $21.41 \pm 0.43$ & $1.81 \pm 0.56$ \\
\hline 46.89 & 4051373 & 6319 & 47.00 & $i$ & $21.15 \pm 0.43$ & $2.86 \pm 0.61$ \\
\hline 50.87 & 4395336 & 3680 & 50.93 & $g$ & $22.11 \pm 0.43$ & $1.12 \pm 0.56$ \\
\hline 50.87 & 4395336 & 3680 & 50.93 & $i$ & $21.28 \pm 0.43$ & $1.64 \pm 0.63$ \\
\hline 51.88 & 4482266 & 5119 & 51.96 & $r$ & $21.41 \pm 0.43$ & $0.83 \pm 0.56$ \\
\hline 51.88 & 4482267 & 5119 & 51.96 & $i$ & $21.20 \pm 0.43$ & $2.08 \pm 0.58$ \\
\hline 51.88 & 4482314 & 4295 & 51.96 & $Y$ & $21.06 \pm 0.43$ & $3.62 \pm 1.61$ \\
\hline 52.90 & 4570263 & 2560 & 52.94 & $i$ & $21.23 \pm 0.43$ & $2.51 \pm 0.74$ \\
\hline 53.88 & 4655540 & 3839 & 53.94 & $r$ & $21.36 \pm 0.43$ & $2.56 \pm 0.63$ \\
\hline 53.88 & 4655540 & 3839 & 53.94 & $i$ & $21.16 \pm 0.43$ & $2.54 \pm 0.64$ \\
\hline 53.88 & 4655589 & 3221 & 53.94 & $Y$ & $21.13 \pm 0.44$ & $2.27 \pm 1.56$ \\
\hline 54.88 & 4741565 & 4800 & 54.96 & $g$ & $21.94 \pm 0.44$ & $1.75 \pm 0.75$ \\
\hline 54.88 & 4741565 & 5119 & 54.96 & $i$ & $21.29 \pm 0.43$ & $1.82 \pm 0.62$ \\
\hline 54.88 & 4741615 & 2147 & 54.96 & $Y$ & $20.74 \pm 0.44$ & $4.30 \pm 3.39$ \\
\hline 56.88 & 4914230 & 5119 & 56.96 & $r$ & $21.54 \pm 0.43$ & $-0.07 \pm 1.27$ \\
\hline 56.88 & 4914230 & 5119 & 56.96 & $i$ & $21.16 \pm 0.43$ & $2.96 \pm 0.72$ \\
\hline 56.88 & 4914278 & 4295 & 56.96 & $Y$ & $20.99 \pm 0.43$ & $1.86 \pm 1.99$ \\
\hline 57.87 & 4999923 & 3839 & 57.93 & $i$ & $21.40 \pm 0.43$ & $0.99 \pm 0.83$ \\
\hline 58.87 & 5086058 & 3839 & 58.92 & $r$ & $21.39 \pm 0.43$ & $1.18 \pm 0.66$ \\
\hline 58.87 & 5086058 & 3839 & 58.92 & $i$ & $21.15 \pm 0.43$ & $2.11 \pm 0.68$ \\
\hline 58.87 & 5086106 & 3221 & 58.92 & $Y$ & $21.03 \pm 0.43$ & $2.65 \pm 1.64$ \\
\hline 59.87 & 5172391 & 3839 & 59.92 & $g$ & $22.19 \pm 0.43$ & $0.50 \pm 0.50$ \\
\hline 59.87 & 5172391 & 3839 & 59.92 & $i$ & $21.36 \pm 0.43$ & $2.19 \pm 0.61$ \\
\hline 65.86 & 5690438 & 2560 & 65.90 & $i$ & $21.33 \pm 0.43$ & $1.71 \pm 0.67$ \\
\hline 65.86 & 5690438 & 2560 & 65.90 & $g$ & $22.20 \pm 0.43$ & $1.00 \pm 0.55$ \\
\hline 66.86 & 5776448 & 2480 & 66.90 & $i$ & $21.43 \pm 0.43$ & $1.61 \pm 0.88$ \\
\hline 66.86 & 5776448 & 2560 & 66.90 & $r$ & $21.51 \pm 0.43$ & $0.06 \pm 0.79$ \\
\hline 215.15 & 18588991 & 8479 & 215.29 & $r$ & $21.60 \pm 0.43$ & $0.20 \pm 0.52$ \\
\hline 215.15 & 18588991 & 8479 & 215.29 & $i$ & $21.35 \pm 0.42$ & $0.27 \pm 0.55$ \\
\hline 217.15 & 18761668 & 8880 & 217.30 & $g$ & $22.26 \pm 0.43$ & $0.10 \pm 0.47$ \\
\hline 217.15 & 18761668 & 8880 & 217.30 & $i$ & $21.40 \pm 0.42$ & $0.12 \pm 0.52$ \\
\hline 217.15 & 18761678 & 3691 & 217.30 & $Z$ & $21.07 \pm 0.43$ & $-0.67 \pm 1.20$ \\
\hline 219.14 & 18933938 & 4093 & 219.30 & $Z$ & $21.17 \pm 0.43$ & $1.71 \pm 1.18$ \\
\hline 219.14 & 18933983 & 9920 & 219.31 & $r$ & $21.57 \pm 0.42$ & $0.12 \pm 0.49$ \\
\hline 219.14 & 18933983 & 9920 & 219.31 & $i$ & $21.41 \pm 0.42$ & $0.63 \pm 0.51$ \\
\hline 221.11 & 19104115 & 6319 & 221.23 & $g$ & $22.25 \pm 0.43$ & $0.33 \pm 0.54$ \\
\hline 221.11 & 19104115 & 6560 & 221.23 & $i$ & $21.46 \pm 0.43$ & $0.31 \pm 0.79$ \\
\hline 223.14 & 19279069 & 10240 & 223.30 & $r$ & $21.61 \pm 0.42$ & $0.27 \pm 0.50$ \\
\hline 223.14 & 19279069 & 10240 & 223.30 & $i$ & $21.45 \pm 0.42$ & $0.83 \pm 0.53$ \\
\hline 223.14 & 19279078 & 4295 & 223.30 & $Z$ & $20.99 \pm 0.43$ & $1.61 \pm 1.32$ \\
\hline 229.14 & 19797449 & 4765 & 229.32 & $Z$ & $21.06 \pm 0.43$ & $-0.32 \pm 1.08$ \\
\hline 229.14 & 19797488 & 11040 & 229.32 & $g$ & $22.26 \pm 0.43$ & $0.18 \pm 0.47$ \\
\hline 229.14 & 19797488 & 11439 & 229.32 & $i$ & $21.47 \pm 0.42$ & $0.67 \pm 0.50$ \\
\hline 231.13 & 19969644 & 10479 & 231.33 & $i$ & $21.38 \pm 0.43$ & $0.65 \pm 0.61$ \\
\hline 231.13 & 19969803 & 10240 & 231.33 & $r$ & $22.18 \pm 0.43$ & $1.05 \pm 0.54$ \\
\hline 303.53 & 26224942 & 28254 & 384.70 & $Y$ & $21.16 \pm 0.43$ & $1.31 \pm 0.75$ \\
\hline 303.53 & 26224942 & 28389 & 384.70 & $Z$ & $21.06 \pm 0.43$ & $-0.51 \pm 0.59$ \\
\hline 303.53 & 26224983 & 27986 & 384.70 & $J$ & $21.36 \pm 0.43$ & $2.10 \pm 0.93$ \\
\hline 303.53 & 26224983 & 28694 & 384.70 & $H$ & $20.86 \pm 0.44$ & $1.64 \pm 1.79$ \\
\hline 306.52 & 26483726 & 79599 & 395.67 & $r$ & $21.58 \pm 0.42$ & $0.26 \pm 0.46$ \\
\hline 314.00 & 27129366 & 145272 & 418.10 & $i$ & $21.44 \pm 0.42$ & $0.01 \pm 0.45$ \\
\hline 353.44 & 30537092 & 53200 & 378.66 & $g$ & $22.25 \pm 0.42$ & $0.10 \pm 0.45$ \\
\hline 1152.86 & 99607079 & 23439 & 1192.87 & $r$ & $21.57 \pm 0.42$ & $0.00 \pm 0.44$ \\
\hline 1153.36 & 99650438 & 63920 & 1194.37 & $i$ & $21.46 \pm 0.42$ & $-0.00 \pm 0.48$ \\
\hline 1153.36 & 99650451 & 28456 & 1194.37 & $H$ & $21.27 \pm 0.44$ & $5.55 \pm 54.54$ \\
\hline 1153.36 & 99650451 & 28657 & 1194.37 & $J$ & $21.30 \pm 0.43$ & $0.11 \pm 1.62$ \\
\hline 1153.36 & 99650553 & 29463 & 1194.37 & $Y$ & $21.34 \pm 0.43$ & $0.00 \pm 0.96$ \\
\hline 1153.36 & 99650553 & 29865 & 1194.37 & $Z$ & $21.21 \pm 0.43$ & $0.05 \pm 0.73$ \\
\hline 1153.85 & 99692829 & 38160 & 1193.88 & $g$ & $22.21 \pm 0.42$ & $0.00 \pm 0.44$ \\
\hline
\end{tabular}


TABLE 7

RESIDUALS BETWEEN OBSERVATIONS AND POWER-LAW MODEL FOR RATIR DATA FOR EARLY AFTERGLOW (BEFORE 0.7 DAYS)

\begin{tabular}{|c|c|c|c|c|c|}
\hline \multirow[t]{2}{*}{$t_{0}(\mathrm{~s})$} & \multicolumn{5}{|c|}{ Filter } \\
\hline & $i[\mu \mathrm{Jy}]$ & $Z[\mu \mathrm{Jy}]$ & $Y[\mu \mathrm{Jy}]$ & $J[\mu \mathrm{Jy}]$ & $H[\mu \mathrm{Jy}]$ \\
\hline 929 & $1099.93 \pm 539.14$ & $\ldots$ & $\ldots$ & $\ldots$ & $\ldots$ \\
\hline 1009 & $\ldots$ & $2805.23 \pm 601.42$ & $\ldots$ & $\ldots$ & $\ldots$ \\
\hline 1012 & $\ldots$ & $\ldots$ & $\ldots$ & $3354.17 \pm 649.69$ & $\ldots$ \\
\hline 1037 & $1373.25 \pm 518.59$ & $\ldots$ & $\ldots$ & $\ldots$ & $\ldots$ \\
\hline 1111 & $\ldots$ & $\ldots$ & $\ldots$ & $\ldots$ & $591.10 \pm 605.02$ \\
\hline 1114 & $\ldots$ & $\ldots$ & $2951.70 \pm 651.46$ & $\ldots$ & $\ldots$ \\
\hline 1140 & $314.13 \pm 467.87$ & $\ldots$ & $\ldots$ & $\ldots$ & $\ldots$ \\
\hline 1216 & $\ldots$ & $\cdots$ & $-497.04 \pm 535.12$ & $\cdots$ & $2238.44 \pm 567.07$ \\
\hline 1249 & $-34.68 \pm 385.64$ & $\ldots$ & $\ldots$ & $\ldots$ & $\ldots$ \\
\hline 1323 & $\ldots$ & $832.70 \pm 441.93$ & $\ldots$ & $\ldots$ & $\ldots$ \\
\hline 1326 & ( & $\ldots$ & $\ldots$ & $1761.38 \pm 465.51$ & $\ldots$ \\
\hline 1348 & $-541.16 \pm 341.65$ & $\ldots$ & $\ldots$ & $\ldots$ & $\ldots$ \\
\hline 1429 & $\ldots$ & $\ldots$ & $\ldots$ & $705.36 \pm 425.84$ & $\ldots$ \\
\hline 1432 & $\ldots$ & $207.34 \pm 388.42$ & $\ldots$ & $\ldots$ & $\ldots$ \\
\hline 1457 & $-341.40 \pm 325.81$ & $\ldots$ & $\ldots$ & $\ldots$ & $\ldots$ \\
\hline 1532 & $\ldots$ & $\ldots$ & $\ldots$ & $\ldots$ & $271.86 \pm 473.29$ \\
\hline 1534 & $\ldots$ & $\ldots$ & $398.85 \pm 421.20$ & $\ldots$ & $\ldots$ \\
\hline 1579 & $-344.62 \pm 303.13$ & $\ldots$ & $\ldots$ & $\ldots$ & $\ldots$ \\
\hline 1653 & $\ldots$ & $\ldots$ & $760.35 \pm 424.83$ & $\ldots$ & $413.36 \pm 387.92$ \\
\hline 1683 & $-10.17 \pm 294.07$ & $\ldots$ & $\ldots$ & $\ldots$ & $\ldots$ \\
\hline 1762 & $\ldots$ & $237.31 \pm 321.87$ & $\ldots$ & $\ldots$ & $\ldots$ \\
\hline 1764 & $\ldots$ & $\ldots$ & $\ldots$ & $362.12 \pm 345.01$ & $\ldots$ \\
\hline 1778 & $-26.80 \pm 276.89$ & $\ldots$ & $\ldots$ & & $\ldots$ \\
\hline 1858 & $\ldots$ & $\ldots$ & $\ldots$ & $702.49 \pm 330.55$ & $\ldots$ \\
\hline 1859 & $\ldots$ & $98.84 \pm 303.47$ & $\ldots$ & $\ldots$ & $\ldots$ \\
\hline 1887 & $-345.25 \pm 280.13$ & $\ldots$ & $\ldots$ & $\ldots$ & $\ldots$ \\
\hline 1962 & $\ldots$ & $\ldots$ & $260.62 \pm 336.24$ & $\ldots$ & $\ldots$ \\
\hline 1964 & $\ldots$ & $\ldots$ & $\ldots$ & $\ldots$ & $843.22 \pm 386.70$ \\
\hline 1993 & $63.20 \pm 253.56$ & $\ldots$ & $\ldots$ & $\ldots$ & $\ldots$ \\
\hline 2067 & $\ldots$ & $\ldots$ & $116.67 \pm 309.69$ & $\ldots$ & $\ldots$ \\
\hline 2068 & $\ldots$ & $\ldots$ & $\ldots$ & $\ldots$ & $810.12 \pm 362.53$ \\
\hline 2120 & $-776.28 \pm 223.10$ & $\ldots$ & $\ldots$ & $\ldots$ & $\ldots$ \\
\hline 2196 & & $1021.73 \pm 282.60$ & $\ldots$ & $-721.88 \pm 256.29$ & $\ldots$ \\
\hline 2223 & $-304.10 \pm 218.44$ & $\ldots$ & $\ldots$ & $\ldots$ & $\ldots$ \\
\hline 2298 & $\ldots$ & $\ldots$ & $\ldots$ & $416.47 \pm 272.67$ & $\ldots$ \\
\hline 2300 & $\ldots$ & $-45.74 \pm 248.32$ & $\ldots$ & $\ldots$ & $\ldots$ \\
\hline 2328 & $11.83 \pm 217.43$ & $\ldots$ & $\ldots$ & $\ldots$ & $\ldots$ \\
\hline 2408 & & $\ldots$ & $365.80 \pm 287.64$ & $\ldots$ & $-10.21 \pm 276.78$ \\
\hline 2422 & $-416.26 \pm 212.59$ & $\cdots$ & $\ldots$ & $\cdots$ & $\ldots$ \\
\hline 2502 & $\ldots$ & $\ldots$ & $-192.37 \pm 258.31$ & $\ldots$ & $\ldots$ \\
\hline 2506 & $\ldots$ & $\ldots$ & $\ldots$ & $\ldots$ & $191.98 \pm 266.82$ \\
\hline 2531 & $-446.33 \pm 184.76$ & $\ldots$ & $\ldots$ & $\ldots$ & $\ldots$ \\
\hline 2605 & $\ldots$ & $\ldots$ & $\ldots$ & $26.02 \pm 236.63$ & $\ldots$ \\
\hline 2607 & $\ldots$ & $-175.13 \pm 217.63$ & $\ldots$ & $\ldots$ & $\ldots$ \\
\hline 2654 & $-305.91 \pm 178.58$ & . & $\ldots$ & $\ldots$ & $\ldots$ \\
\hline 2729 & $\ldots$ & $\ldots$ & $\cdots$ & $-206.29 \pm 217.49$ & $\cdots$ \\
\hline 2732 & $\ldots$ & $-567.47 \pm 204.66$ & $\cdots$ & . & $\cdots$ \\
\hline 2780 & $-522.44 \pm 193.74$ & $\ldots$ & $\ldots$ & $\ldots$ & $\ldots$ \\
\hline 2855 & $\ldots$ & $\ldots$ & $-77.25 \pm 243.18$ & $\ldots$ & $\ldots$ \\
\hline 2856 & $\ldots$ & $\ldots$ & $\ldots$ & $\ldots$ & $106.19 \pm 273.15$ \\
\hline 2884 & $-204.39 \pm 164.74$ & $\ldots$ & $\ldots$ & $\ldots$ & $\ldots$ \\
\hline 2960 & $\ldots$ & $\ldots$ & $-147.92 \pm 218.22$ & $\ldots$ & $\ldots$ \\
\hline 2961 & $\ldots$ & $\ldots$ & $\ldots$ & $\ldots$ & $605.39 \pm 291.40$ \\
\hline 2990 & $-278.80 \pm 162.89$ & $\ldots$ & $\ldots$ & $\ldots$ & $\ldots$ \\
\hline 3067 & $\ldots$ & $-239.20 \pm 178.90$ & $\ldots$ & $\ldots$ & $\ldots$ \\
\hline 3069 & $\ldots$ & $\ldots$ & $\ldots$ & $-166.34 \pm 196.09$ & $\ldots$ \\
\hline 3108 & $-360.19 \pm 150.44$ & $\ldots$ & $\ldots$ & $\ldots$ & $\ldots$ \\
\hline 3186 & .. & $77.07 \pm 182.57$ & $\ldots$ & $-1002.49 \pm 167.46$ & $\ldots$ \\
\hline 3213 & $-362.91 \pm 150.66$ & $\ldots$ & $\ldots$ & $\ldots$ & $\cdots$ \\
\hline 3292 & $\ldots$ & $\ldots$ & $-227.89 \pm 191.97$ & $\ldots$ & $\ldots$ \\
\hline 3293 & $\ldots$ & $\ldots$ & . & $\ldots$ & $329.50 \pm 284.11$ \\
\hline 3307 & $-333.22 \pm 144.97$ & $\ldots$ & $\ldots$ & $\ldots$ & $\ldots$ \\
\hline 3387 & - & $\cdots$ & $\ldots$ & $\cdots$ & $247.13 \pm 256.03$ \\
\hline 3389 & & $\cdots$ & $-131.45 \pm 190.18$ & $\cdots$ & $\ldots$ \\
\hline 3418 & $-168.13 \pm 156.71$ & $\ldots$ & $\ldots$ & $\ldots$ & $\cdots$ \\
\hline 3493 & & $-169.05 \pm 185.52$ & $\ldots$ & $-868.70 \pm 156.80$ & $\cdots$ \\
\hline 3535 & $-105.29 \pm 140.17$ & & $\ldots$ & $\ldots$ & $\ldots$ \\
\hline 3610 & ‥ & $-128.75 \pm 158.66$ & $\ldots$ & $\ldots$ & $\ldots$ \\
\hline 3611 & $\ldots$ & $\ldots$ & $\ldots$ & $-100.35 \pm 168.94$ & $\ldots$ \\
\hline 3640 & $-217.72 \pm 133.67$ & $\cdots$ & $\cdots$ & . & $\ldots$ \\
\hline 3719 & $\ldots$ & $\cdots$ & $\ldots$ & $\cdots$ & $491.39 \pm 228.38$ \\
\hline 3721 & $\ldots$ & $\cdots$ & $11.29 \pm 175.04$ & $\ldots$ & $\ldots$ \\
\hline 3735 & $-143.56 \pm 143.25$ & $\ldots$ & . & $\cdots$ & $\cdots$ \\
\hline
\end{tabular}


TABLE 7 - Continued

\begin{tabular}{|c|c|c|c|c|c|}
\hline \multirow[t]{2}{*}{$t_{0}(\mathrm{~s})$} & \multicolumn{5}{|c|}{ Filter } \\
\hline & $i[\mu \mathrm{Jy}]$ & $Z[\mu \mathrm{Jy}]$ & $Y[\mu \mathrm{Jy}]$ & $J[\mu \mathrm{Jy}]$ & $H[\mu \mathrm{Jy}]$ \\
\hline 3814 & $\ldots$ & $\ldots$ & $-144.77 \pm 170.13$ & $\ldots$ & $\ldots$ \\
\hline 3815 & $\ldots$ & $\ldots$ & .. & $\cdots$ & $476.88 \pm 207.06$ \\
\hline 3842 & $-116.80 \pm 128.28$ & $\ldots$ & $\ldots$ & $\ldots$ & $\ldots$ \\
\hline 3918 & $\ldots$ & $\ldots$ & $\cdots$ & $-245.69 \pm 183.08$ & $\cdots$ \\
\hline 3919 & $\ldots$ & $-282.94 \pm 158.48$ & $\ldots$ & $\ldots$ & $\ldots$ \\
\hline 3963 & $-22.03 \pm 123.97$ & ... & $\cdots$ & $\ldots$ & $\ldots$ \\
\hline 4038 & $\ldots$ & $429.42 \pm 154.41$ & $\cdots$ & $-334.68 \pm 144.00$ & $\cdots$ \\
\hline 4069 & $-204.18 \pm 131.80$ & $\ldots$ & $\cdots$ & $\ldots$ & $\ldots$ \\
\hline 4145 & . & $\cdots$ & $\ldots$ & $\cdots$ & $294.22 \pm 210.48$ \\
\hline 4146 & $\ldots$ & $\ldots$ & $88.92 \pm 159.62$ & $\ldots$ & $\ldots$ \\
\hline 4171 & $-43.37 \pm 129.77$ & $\ldots$ & $\ldots$ & $\ldots$ & $\ldots$ \\
\hline 4247 & $\ldots$ & $\ldots$ & $\ldots$ & $\ldots$ & $403.42 \pm 206.94$ \\
\hline 4248 & $\ldots$ & $\cdots$ & $92.01 \pm 155.91$ & $\cdots$ & $\ldots$ \\
\hline 4295 & $-68.97 \pm 114.90$ & $\ldots$ & $\ldots$ & $\ldots$ & $\cdots$ \\
\hline 4371 & $\ldots$ & $430.77 \pm 141.70$ & $\cdots$ & $-268.65 \pm 134.35$ & $\cdots$ \\
\hline 4414 & $20.95 \pm 113.41$ & & $\ldots$ & $\ldots$ & $\ldots$ \\
\hline 4493 & $\ldots$ & $405.50 \pm 142.94$ & $\cdots$ & $-305.07 \pm 129.43$ & $\cdots$ \\
\hline 4523 & $-753.30 \pm 142.08$ & $\ldots$ & $\ldots$ & $\ldots$ & $\ldots$ \\
\hline 4598 & $\ldots$ & $\cdots$ & $-62.47 \pm 384.04$ & $\cdots$ & $-379.43 \pm 186.04$ \\
\hline 4642 & $128.93 \pm 111.30$ & $\cdots$ & $\ldots$ & $\cdots$ & $\ldots$ \\
\hline 4718 & $\ldots$ & $\cdots$ & $68.32 \pm 145.11$ & $\cdots$ & $\ldots$ \\
\hline 4719 & $\ldots$ & $\ldots$ & $\ldots$ & $\ldots$ & $193.53 \pm 181.91$ \\
\hline 4742 & $13.53 \pm 106.79$ & $\ldots$ & $\cdots$ & $\ldots$ & $\ldots$ \\
\hline 4821 & $\ldots$ & $\ldots$ & $\ldots$ & $-34.60 \pm 133.02$ & $\ldots$ \\
\hline 4822 & $\ldots$ & $-10.51 \pm 122.19$ & $\cdots$ & $\cdots$ & $\cdots$ \\
\hline 4846 & $3.86 \pm 107.35$ & $\ldots$ & $\cdots$ & $\ldots$ & $\cdots$ \\
\hline 4922 & $\ldots$ & $\ldots$ & $\cdots$ & $16.76 \pm 130.56$ & $\cdots$ \\
\hline 4923 & $\ldots$ & $-1574.14 \pm 86.59$ & $\ldots$ & $\ldots$ & $\ldots$ \\
\hline 4953 & $12.21 \pm 106.93$ & $\ldots$ & $\ldots$ & $\ldots$ & $\ldots$ \\
\hline 5028 & $\ldots$ & $\ldots$ & $\ldots$ & $\ldots$ & $826.11 \pm 322.54$ \\
\hline 5032 & $\ldots$ & $\ldots$ & $216.10 \pm 150.56$ & $\cdots$ & $\cdots$ \\
\hline 5059 & $33.37 \pm 106.53$ & $\cdots$ & $\ldots$ & $\cdots$ & $\ldots$ \\
\hline 5134 & $\ldots$ & $\cdots$ & $\ldots$ & $\ldots$ & $422.64 \pm 177.33$ \\
\hline 5135 & $\ldots$ & $\ldots$ & $270.93 \pm 131.95$ & $\ldots$ & $\ldots$ \\
\hline 5186 & $-59.76 \pm 133.88$ & $\ldots$ & $\ldots$ & $\ldots$ & $\ldots$ \\
\hline 5260 & $\ldots$ & $\ldots$ & $\ldots$ & $107.47 \pm 126.22$ & $\ldots$ \\
\hline 5262 & $\ldots$ & $139.56 \pm 115.97$ & $\ldots$ & $\ldots$ & $\ldots$ \\
\hline 5287 & $187.44 \pm 103.88$ & & $\cdots$ & & $\ldots$ \\
\hline 5362 & $\ldots$ & $402.54 \pm 128.18$ & $\cdots$ & $-101.23 \pm 111.83$ & $\cdots$ \\
\hline 5394 & $48.19 \pm 97.91$ & $\ldots$ & $\ldots$ & $\ldots$ & $\ldots$ \\
\hline 5470 & $\ldots$ & $\ldots$ & $732.22 \pm 209.78$ & $\ldots$ & $207.25 \pm 125.30$ \\
\hline 5506 & $22.50 \pm 97.93$ & $\ldots$ & $\ldots$ & $\ldots$ & $\ldots$ \\
\hline 5586 & $\ldots$ & $\ldots$ & $605.06 \pm 160.57$ & $\ldots$ & $124.24 \pm 126.73$ \\
\hline 5615 & $165.21 \pm 94.75$ & $\cdots$ & $\ldots$ & $\ldots$ & $\ldots$ \\
\hline 5690 & $\ldots$ & $\ldots$ & $\ldots$ & $255.73 \pm 118.96$ & $\cdots$ \\
\hline 5694 & $\ldots$ & $-29.63 \pm 109.85$ & $\cdots$ & $\ldots$ & $\cdots$ \\
\hline 5715 & $176.93 \pm 92.38$ & $\ldots$ & $\ldots$ & $\ldots$ & $\ldots$ \\
\hline 5789 & $\ldots$ & $\ldots$ & $\ldots$ & $338.34 \pm 120.32$ & $\ldots$ \\
\hline 5790 & $\ldots$ & $224.85 \pm 106.05$ & $\ldots$ & $\ldots$ & $\ldots$ \\
\hline 5823 & $203.43 \pm 90.44$ & $\ldots$ & $\ldots$ & $\ldots$ & $\ldots$ \\
\hline 5897 & $\ldots$ & $\cdots$ & $\ldots$ & $\cdots$ & $557.04 \pm 129.97$ \\
\hline 5898 & $\ldots$ & $\cdots$ & $112.54 \pm 123.03$ & $\cdots$ & $\ldots$ \\
\hline 5942 & $-57.18 \pm 99.26$ & $\ldots$ & $\ldots$ & $\ldots$ & $\ldots$ \\
\hline 6018 & ... & $\cdots$ & $240.91 \pm 114.64$ & $\ldots$ & $\ldots$ \\
\hline 6020 & $\ldots$ & $\ldots$ & $\ldots$ & $\ldots$ & $782.68 \pm 182.53$ \\
\hline 6050 & $120.56 \pm 87.90$ & $\cdots$ & $\cdots$ & $\ldots$ & $\cdots$ \\
\hline 6124 & $\ldots$ & $\ldots$ & $\cdots$ & $236.29 \pm 108.81$ & $\cdots$ \\
\hline 6125 & $\cdots$ & $138.35 \pm 99.38$ & $\cdots$ & $\ldots$ & $\cdots$ \\
\hline
\end{tabular}

TABLE 8

RESIDUALS BETWEEN OBSERVATIONS AND POWER-LAW MODEL FOR RATIR DATA FOR LATE AFTERGLOW (AFTER 0.7 DAYS)

\begin{tabular}{|c|c|c|c|c|c|c|c|}
\hline \multirow[t]{2}{*}{$t_{0}(\mathrm{~d})$} & \multicolumn{7}{|c|}{ Filter } \\
\hline & $g[\mu \mathrm{Jy}]$ & $r[\mu \mathrm{Jy}]$ & $i[\mu \mathrm{Jy}]$ & $Z[\mu \mathrm{Jy}]$ & $Y[\mu \mathrm{Jy}]$ & $J[\mu \mathrm{Jy}]$ & $H[\mu \mathrm{Jy}]$ \\
\hline 0.83 & $\cdots$ & $-23.90 \pm 3.62$ & $-37.66 \pm 3.51$ & $-48.43 \pm 9.07$ & $-50.50 \pm 9.44$ & $-66.74 \pm 10.87$ & $-54.80 \pm 10.22$ \\
\hline 0.85 & $\cdots$ & $-20.88 \pm 4.07$ & $-38.87 \pm 3.49$ & $-46.55 \pm 9.88$ & $-31.91 \pm 10.33$ & $-56.30 \pm 9.97$ & $-46.25 \pm 17.28$ \\
\hline 0.87 & $\ldots$ & $-21.47 \pm 2.53$ & $-25.19 \pm 3.31$ & $-41.10 \pm 7.91$ & $-40.88 \pm 9.58$ & $-29.30 \pm 9.93$ & $-41.91 \pm 9.72$ \\
\hline 0.90 & $\ldots$ & $-15.79 \pm 4.17$ & $-32.39 \pm 3.86$ & $-39.28 \pm 8.21$ & $-56.57 \pm 7.43$ & $-39.85 \pm 8.11$ & $-50.19 \pm 7.82$ \\
\hline 0.92 & $\ldots$ & $-22.70 \pm 2.79$ & $-28.13 \pm 3.29$ & $-40.63 \pm 7.88$ & $-26.98 \pm 7.88$ & $-69.72 \pm 7.39$ & $-14.33 \pm 8.76$ \\
\hline
\end{tabular}


TABLE $8-$ Continued

\begin{tabular}{|c|c|c|c|c|c|c|c|}
\hline \multirow[t]{2}{*}{$t_{0}(\mathrm{~d})$} & \multicolumn{7}{|c|}{ Filter } \\
\hline & $g[\mu \mathrm{Jy}]$ & $r[\mu \mathrm{Jy}]$ & $i[\mu \mathrm{Jy}]$ & $Z[\mu \mathrm{Jy}]$ & $Y[\mu \mathrm{Jy}]$ & $J[\mu \mathrm{Jy}]$ & $H[\mu \mathrm{Jy}]$ \\
\hline 0.94 & $\ldots$ & $-19.90 \pm 2.81$ & $-32.23 \pm 3.14$ & $-35.16 \pm 5.39$ & $-37.45 \pm 8.05$ & $-30.91 \pm 8.56$ & $14.00 \pm 8.31$ \\
\hline 0.97 & $\ldots$ & $-14.07 \pm 3.22$ & $-24.73 \pm 3.38$ & $-26.06 \pm 6.43$ & $-28.58 \pm 7.58$ & $-35.32 \pm 7.58$ & $-7.64 \pm 7.58$ \\
\hline 0.99 & $\ldots$ & $-12.95 \pm 3.62$ & $-25.80 \pm 2.90$ & $-22.56 \pm 7.51$ & $-42.19 \pm 6.59$ & $-38.27 \pm 7.29$ & $10.01 \pm 7.96$ \\
\hline 1.01 & $\ldots$ & $-7.59 \pm 3.37$ & $-26.91 \pm 3.13$ & $-21.96 \pm 7.38$ & $-30.36 \pm 7.26$ & $-21.45 \pm 7.40$ & $-17.75 \pm 7.80$ \\
\hline 1.04 & $\ldots$ & $-10.58 \pm 3.01$ & $-20.13 \pm 3.02$ & $-18.67 \pm 9.83$ & $-30.09 \pm 7.18$ & $-22.21 \pm 8.67$ & $-25.60 \pm 10.30$ \\
\hline 1.06 & $\ldots$ & $-15.91 \pm 3.58$ & $-19.59 \pm 3.08$ & $-40.60 \pm 8.52$ & $-25.47 \pm 7.58$ & $-16.84 \pm 9.29$ & $-5.48 \pm 10.43$ \\
\hline 1.83 & $\cdots$ & $-1.14 \pm 1.17$ & $-2.56 \pm 1.76$ & $3.03 \pm 4.45$ & $6.59 \pm 4.86$ & $-8.12 \pm 7.28$ & $10.77 \pm 8.73$ \\
\hline 1.85 & $\ldots$ & $-2.12 \pm 1.08$ & $-2.66 \pm 1.67$ & $-5.28 \pm 3.94$ & $5.02 \pm 5.26$ & $-3.83 \pm 6.66$ & $0.36 \pm 8.23$ \\
\hline 1.87 & $\ldots$ & $-1.21 \pm 1.19$ & $-1.91 \pm 1.62$ & $-7.42 \pm 4.18$ & $4.85 \pm 5.26$ & $-7.31 \pm 6.44$ & $12.60 \pm 8.85$ \\
\hline 1.89 & $\ldots$ & $0.36 \pm 0.86$ & $-2.55 \pm 1.24$ & $-0.80 \pm 3.64$ & $1.51 \pm 4.21$ & $-9.33 \pm 5.33$ & $21.06 \pm 7.82$ \\
\hline 1.91 & $\ldots$ & $-2.19 \pm 1.04$ & $-2.26 \pm 1.60$ & $-1.96 \pm 3.56$ & $3.04 \pm 4.33$ & $-4.08 \pm 5.43$ & $3.01 \pm 6.82$ \\
\hline 1.93 & $\ldots$ & $-1.24 \pm 1.32$ & $-2.41 \pm 1.59$ & $-0.62 \pm 3.54$ & $0.67 \pm 4.26$ & $-3.65 \pm 5.17$ & $16.87 \pm 7.51$ \\
\hline 1.96 & $\ldots$ & $0.51 \pm 1.52$ & $-1.73 \pm 1.48$ & $2.56 \pm 3.65$ & $4.93 \pm 4.65$ & $-3.31 \pm 7.80$ & $19.87 \pm 6.70$ \\
\hline 1.98 & $\ldots$ & $-1.38 \pm 1.62$ & $-0.24 \pm 1.41$ & $-5.77 \pm 3.98$ & $13.89 \pm 4.77$ & $1.87 \pm 5.53$ & $43.92 \pm 10.52$ \\
\hline 2.00 & $\ldots$ & $-2.54 \pm 1.24$ & $1.08 \pm 1.59$ & $6.36 \pm 4.22$ & $-3.77 \pm 4.65$ & $-5.57 \pm 5.55$ & $35.66 \pm 7.94$ \\
\hline 2.02 & $\ldots$ & $-2.07 \pm 1.33$ & $0.57 \pm 1.47$ & & $\ldots$ & $5.51 \pm 6.66$ & $10.72 \pm 9.75$ \\
\hline 2.03 & $\ldots$ & & & $3.21 \pm 4.22$ & $-3.50 \pm 5.05$ & & \\
\hline 2.04 & $\ldots$ & $-0.80 \pm 1.60$ & $-1.18 \pm 1.93$ & & & $5.76 \pm 7.21$ & $31.37 \pm 11.22$ \\
\hline 2.83 & $\cdots$ & $0.04 \pm 0.68$ & $-1.22 \pm 1.01$ & $5.82 \pm 2.89$ & $13.99 \pm 4.88$ & $-3.86 \pm 6.33$ & $22.36 \pm 8.99$ \\
\hline 2.85 & $\ldots$ & $1.91 \pm 1.01$ & $0.51 \pm 1.14$ & $-0.35 \pm 3.12$ & $10.05 \pm 4.87$ & $2.82 \pm 7.44$ & $5.39 \pm 11.54$ \\
\hline 2.87 & $\ldots$ & $2.22 \pm 0.70$ & $0.25 \pm 0.99$ & $3.65 \pm 2.81$ & $17.59 \pm 5.00$ & $-1.42 \pm 5.62$ & $19.63 \pm 8.72$ \\
\hline 2.89 & $\ldots$ & $1.50 \pm 0.81$ & $0.05 \pm 0.88$ & $5.80 \pm 2.37$ & $-2.79 \pm 3.37$ & $-6.73 \pm 4.70$ & $6.42 \pm 6.93$ \\
\hline 2.91 & $\ldots$ & $0.41 \pm 0.78$ & $-0.30 \pm 0.93$ & $-2.00 \pm 2.60$ & $2.25 \pm 3.87$ & $-1.02 \pm 4.80$ & $9.47 \pm 7.36$ \\
\hline 2.93 & $\ldots$ & $0.20 \pm 0.96$ & $-0.41 \pm 0.94$ & $-0.22 \pm 2.62$ & $2.15 \pm 5.01$ & $2.52 \pm 4.73$ & $23.24 \pm 7.61$ \\
\hline 2.95 & $\ldots$ & $0.07 \pm 0.97$ & $-0.34 \pm 0.97$ & $4.08 \pm 2.62$ & $10.02 \pm 4.21$ & $6.05 \pm 4.63$ & $14.62 \pm 7.89$ \\
\hline 2.97 & $\ldots$ & $-0.39 \pm 0.81$ & $-0.72 \pm 0.98$ & $-0.90 \pm 2.91$ & $5.18 \pm 4.19$ & $-0.48 \pm 4.68$ & $32.10 \pm 8.50$ \\
\hline 2.99 & $\ldots$ & $0.64 \pm 0.94$ & $2.30 \pm 1.04$ & $-2.29 \pm 2.99$ & $9.74 \pm 4.75$ & $2.42 \pm 5.21$ & $8.66 \pm 9.56$ \\
\hline 3.02 & $\ldots$ & $-2.13 \pm 0.81$ & $3.50 \pm 1.16$ & $0.04 \pm 2.88$ & $9.46 \pm 4.54$ & $6.81 \pm 5.12$ & $20.49 \pm 9.20$ \\
\hline 3.04 & $\ldots$ & $2.05 \pm 1.00$ & $-0.43 \pm 1.02$ & $0.30 \pm 3.10$ & $4.02 \pm 4.53$ & $0.98 \pm 5.47$ & $21.31 \pm 9.52$ \\
\hline 3.82 & $\ldots$ & $-0.42 \pm 0.82$ & $0.05 \pm 0.87$ & $\ldots$ & $\ldots$ & $\ldots$ & $\ldots$ \\
\hline 3.84 & $\ldots$ & $1.05 \pm 0.72$ & $1.35 \pm 0.80$ & $\cdots$ & $\cdots$ & $\cdots$ & $\cdots$ \\
\hline 3.86 & $\ldots$ & $1.65 \pm 0.67$ & $0.46 \pm 0.69$ & $\ldots$ & $\cdots$ & $\cdots$ & $\ldots$ \\
\hline 3.88 & $\ldots$ & $0.38 \pm 0.63$ & $0.40 \pm 0.76$ & $\ldots$ & $\ldots$ & $\ldots$ & $\ldots$ \\
\hline 3.90 & $\ldots$ & $0.15 \pm 0.75$ & $-0.69 \pm 0.74$ & $\ldots$ & $\ldots$ & $\ldots$ & $\ldots$ \\
\hline 3.92 & $\ldots$ & $1.34 \pm 0.67$ & $0.73 \pm 0.80$ & $\ldots$ & $\ldots$ & $\ldots$ & $\ldots$ \\
\hline 3.94 & $\ldots$ & $0.52 \pm 0.76$ & $0.61 \pm 0.75$ & $\ldots$ & $\ldots$ & $\ldots$ & $\ldots$ \\
\hline 3.96 & $\ldots$ & $0.13 \pm 0.62$ & $0.59 \pm 0.68$ & $\ldots$ & $\ldots$ & $\ldots$ & $\ldots$ \\
\hline 3.97 & $\ldots$ & $1.37 \pm 0.69$ & $2.55 \pm 0.82$ & $\ldots$ & $\ldots$ & $\ldots$ & $\ldots$ \\
\hline 4.00 & $\cdots$ & $0.42 \pm 0.65$ & $0.31 \pm 0.66$ & $\cdots$ & $\cdots$ & $\cdots$ & $\cdots$ \\
\hline 4.01 & $\ldots$ & $2.02 \pm 0.71$ & $2.76 \pm 0.75$ & $\ldots$ & $\ldots$ & $\ldots$ & $\ldots$ \\
\hline 4.03 & $\ldots$ & $0.88 \pm 0.75$ & $0.02 \pm 0.86$ & $\ldots$ & $\ldots$ & $\ldots$ & $\ldots$ \\
\hline 5.03 & $-1.51 \pm 0.81$ & $-2.30 \pm 0.95$ & $1.03 \pm 0.84$ & $\ldots$ & $\ldots$ & $\ldots$ & $\ldots$ \\
\hline 5.94 & $\ldots$ & $1.83 \pm 0.33$ & & $\ldots$ & & $\ldots$ & \\
\hline 5.95 & $\ldots$ & $\ldots$ & $1.78 \pm 0.35$ & $0.08 \pm 0.90$ & $1.67 \pm 1.29$ & $7.04 \pm 1.60$ & $3.14 \pm 2.62$ \\
\hline 5.97 & $-0.46 \pm 0.24$ & $\cdots$ & & & & & \\
\hline 6.93 & $0.53 \pm 0.22$ & $\cdots$ & $1.75 \pm 0.33$ & $3.24 \pm 0.89$ & $1.94 \pm 1.35$ & $8.00 \pm 2.36$ & $0.91 \pm 2.76$ \\
\hline 7.94 & $0.45 \pm 0.26$ & $\ldots$ & $1.42 \pm 0.35$ & & & & \\
\hline 7.95 & - & $\ldots$ & & $2.07 \pm 1.01$ & $5.42 \pm 1.54$ & $10.57 \pm 2.09$ & $24.92 \pm 3.36$ \\
\hline 9.96 & $0.51 \pm 0.23$ & $\ldots$ & $2.37 \pm 0.36$ & $3.07 \pm 1.16$ & $3.25 \pm 1.72$ & $2.34 \pm 2.31$ & $-20.23 \pm 3.90$ \\
\hline 10.91 & & $2.27 \pm 0.90$ & & & & & \\
\hline 10.93 & $0.22 \pm 0.62$ & & $2.48 \pm 0.38$ & $0.63 \pm 1.11$ & $3.45 \pm 1.77$ & $9.80 \pm 2.97$ & \\
\hline 11.93 & $1.31 \pm 0.23$ & $2.76 \pm 0.29$ & $2.69 \pm 0.23$ & $2.05 \pm 0.67$ & $1.99 \pm 1.01$ & $-0.66 \pm 1.35$ & $3.62 \pm 2.13$ \\
\hline 12.91 & $1.68 \pm 0.23$ & $3.14 \pm 0.31$ & $3.28 \pm 0.26$ & $3.37 \pm 0.76$ & $4.80 \pm 1.19$ & $5.42 \pm 1.69$ & $-10.94 \pm 2.49$ \\
\hline 13.91 & $1.77 \pm 0.21$ & $3.02 \pm 0.32$ & $2.67 \pm 0.24$ & $1.70 \pm 0.73$ & $3.44 \pm 1.17$ & $8.17 \pm 1.60$ & $-11.87 \pm 2.51$ \\
\hline 14.90 & $\ldots$ & $3.59 \pm 0.30$ & $\ldots$ & & & & \\
\hline 14.91 & $\ldots$ & $\ldots$ & $3.15 \pm 0.23$ & $2.43 \pm 0.67$ & $4.01 \pm 1.01$ & $6.37 \pm 1.39$ & $5.74 \pm 2.11$ \\
\hline 14.92 & $1.89 \pm 0.21$ & $\ldots$ & $\ldots$ & $\ldots$ & $\ldots$ & $\ldots$ & $\ldots$ \\
\hline 15.89 & $\ldots$ & $3.18 \pm 0.33$ & $\ldots$ & $\ldots$ & $\ldots$ & $\ldots$ & $\ldots$ \\
\hline 15.90 & $\ldots$ & $\ldots$ & $2.86 \pm 0.25$ & $2.86 \pm 0.77$ & $2.85 \pm 1.14$ & $0.35 \pm 1.55$ & $-4.27 \pm 2.23$ \\
\hline 15.91 & $2.21 \pm 0.25$ & & & & & & \\
\hline 16.91 & $2.23 \pm 0.28$ & $4.15 \pm 0.36$ & $3.52 \pm 0.28$ & $3.38 \pm 0.82$ & $2.10 \pm 1.45$ & $0.30 \pm 1.85$ & $\ldots$ \\
\hline 17.91 & $\ldots$ & $3.91 \pm 0.41$ & & & & $\ldots$ & $\ldots$ \\
\hline 17.93 & $\ldots$ & $\ldots$ & $3.77 \pm 0.33$ & $2.45 \pm 0.97$ & $0.61 \pm 1.57$ & $\ldots$ & $\ldots$ \\
\hline 17.94 & $1.98 \pm 0.34$ & & & & & $\ldots$ & $\cdots$ \\
\hline 18.92 & $1.94 \pm 0.25$ & $3.12 \pm 0.31$ & $3.55 \pm 0.25$ & $4.14 \pm 0.70$ & $4.46 \pm 1.12$ & $6.55 \pm 1.47$ & $\ldots$ \\
\hline 19.91 & $1.91 \pm 0.26$ & $3.26 \pm 0.37$ & $3.60 \pm 0.28$ & $3.51 \pm 0.80$ & $3.64 \pm 1.34$ & $1.20 \pm 1.55$ & $\cdots$ \\
\hline 20.91 & $\ldots$ & $3.40 \pm 0.42$ & $3.91 \pm 0.34$ & $\ldots$ & $\ldots$ & $\ldots$ & $\ldots$ \\
\hline 20.92 & $1.21 \pm 0.36$ & $\ldots$ & & $5.55 \pm 0.96$ & $3.31 \pm 1.38$ & $1.71 \pm 1.84$ & $\ldots$ \\
\hline 21.92 & $1.24 \pm 0.37$ & $3.10 \pm 0.42$ & $3.47 \pm 0.37$ & $5.00 \pm 1.11$ & $2.32 \pm 1.35$ & $-0.34 \pm 1.68$ & $\ldots$ \\
\hline 22.91 & $\ldots$ & $3.35 \pm 0.39$ & & $\ldots$ & $\ldots$ & $\ldots$ & $\ldots$ \\
\hline 22.92 & $\ldots$ & $\ldots$ & $3.45 \pm 0.27$ & $\cdots$ & $\ldots$ & $\ldots$ & $\ldots$ \\
\hline 22.93 & $2.34 \pm 0.30$ & $\ldots$ & & $\ldots$ & $\ldots$ & $\ldots$ & $\ldots$ \\
\hline 23.91 & $2.17 \pm 0.39$ & $3.15 \pm 0.47$ & $3.69 \pm 0.31$ & $\ldots$ & $\ldots$ & $\cdots$ & $\ldots$ \\
\hline 24.99 & $\ldots$ & $-0.16 \pm 1.02$ & $3.45 \pm 1.08$ & $\ldots$ & $\ldots$ & $\ldots$ & $\ldots$ \\
\hline 25.90 & $\ldots$ & $2.42 \pm 0.51$ & & $\cdots$ & $\cdots$ & $\ldots$ & $\ldots$ \\
\hline
\end{tabular}


TABLE 8 - Continued

\begin{tabular}{|c|c|c|c|c|c|c|c|}
\hline \multirow[t]{2}{*}{$t_{0}(\mathrm{~d})$} & \multicolumn{7}{|c|}{ Filter } \\
\hline & $g[\mu \mathrm{Jy}]$ & $r[\mu \mathrm{Jy}]$ & $i[\mu \mathrm{Jy}]$ & $Z[\mu \mathrm{Jy}]$ & $Y[\mu \mathrm{Jy}]$ & $J[\mu \mathrm{Jy}]$ & $H[\mu \mathrm{Jy}]$ \\
\hline 25.91 & & $\ldots$ & $3.61 \pm 0.38$ & $-4.18 \pm 1.03$ & $2.79 \pm 1.60$ & $\ldots$ & $\ldots$ \\
\hline 25.92 & $1.59 \pm 0.48$ & $\cdots$ & $\ldots$ & $\ldots$ & $\ldots$ & $\ldots$ & $\ldots$ \\
\hline 26.91 & $-2.20 \pm 0.00$ & $\ldots$ & $\ldots$ & $\ldots$ & & $\ldots$ & $\ldots$ \\
\hline 26.92 & $\ldots$ & $2.28 \pm 0.48$ & $4.08 \pm 0.36$ & $3.20 \pm 1.06$ & $-0.28 \pm 1.46$ & $7.28 \pm 1.94$ & $\ldots$ \\
\hline 27.90 & $\ldots$ & $3.38 \pm 0.55$ & $\ldots$ & $\ldots$ & & $\ldots$ & $\ldots$ \\
\hline 27.91 & $\ldots$ & $\ldots$ & $3.24 \pm 0.37$ & $-2.79 \pm 0.92$ & $0.36 \pm 1.43$ & $\ldots$ & $\ldots$ \\
\hline 27.92 & $1.29 \pm 0.54$ & $\ldots$ & $\ldots$ & $\ldots$ & $\ldots$ & $\ldots$ & $\ldots$ \\
\hline 28.90 & $\ldots$ & $1.89 \pm 0.44$ & $\ldots$ & $\ldots$ & $\ldots$ & $\ldots$ & $\ldots$ \\
\hline 28.91 & $\ldots$ & $\ldots$ & $3.58 \pm 0.33$ & $-3.49 \pm 0.86$ & $1.02 \pm 1.28$ & $\ldots$ & $\ldots$ \\
\hline 28.92 & $1.35 \pm 0.43$ & $\ldots$ & $\ldots$ & $\ldots$ & $\ldots$ & $\ldots$ & $\ldots$ \\
\hline 29.92 & $\ldots$ & $2.69 \pm 0.47$ & $\ldots$ & $\ldots$ & $\ldots$ & $\ldots$ & $\ldots$ \\
\hline 29.93 & $\ldots$ & $\ldots$ & $2.77 \pm 0.39$ & $-4.62 \pm 1.08$ & $\ldots$ & $\ldots$ & $\ldots$ \\
\hline 29.94 & $0.93 \pm 0.43$ & $\ldots$ & & $\ldots$ & $\ldots$ & $\cdots$ & $\cdots$ \\
\hline 30.91 & $0.54 \pm 0.44$ & $0.75 \pm 0.44$ & $3.01 \pm 0.36$ & $0.75 \pm 0.99$ & $-0.19 \pm 1.52$ & $\ldots$ & $\ldots$ \\
\hline 31.90 & $0.80 \pm 0.30$ & $0.88 \pm 0.36$ & $2.91 \pm 0.32$ & $\ldots$ & $\ldots$ & $\ldots$ & $\ldots$ \\
\hline 31.91 & & $\ldots$ & $\ldots$ & $0.64 \pm 1.01$ & $3.11 \pm 1.50$ & $\ldots$ & $\ldots$ \\
\hline 32.90 & $0.55 \pm 0.33$ & $\ldots$ & $\ldots$ & $\ldots$ & $\ldots$ & $\ldots$ & $\ldots$ \\
\hline 32.91 & $\ldots$ & $0.83 \pm 0.40$ & $3.04 \pm 0.37$ & $0.13 \pm 1.13$ & $\ldots$ & $\ldots$ & $\ldots$ \\
\hline 33.90 & $0.75 \pm 0.21$ & & & $\ldots$ & $\ldots$ & $\ldots$ & $\ldots$ \\
\hline 33.91 & $\ldots$ & $1.06 \pm 0.70$ & $2.70 \pm 0.23$ & $\ldots$ & $\cdots$ & $\cdots$ & $\ldots$ \\
\hline 34.89 & $0.70 \pm 0.21$ & $\ldots$ & $\ldots$ & $\ldots$ & $\ldots$ & $\ldots$ & $\ldots$ \\
\hline 34.90 & $\ldots$ & $\ldots$ & $2.67 \pm 0.24$ & $\ldots$ & $\ldots$ & $\ldots$ & $\ldots$ \\
\hline 35.90 & $0.11 \pm 0.22$ & $1.57 \pm 1.07$ & $2.28 \pm 0.22$ & $\ldots$ & $\ldots$ & $\ldots$ & $\ldots$ \\
\hline 36.89 & $\ldots$ & $-2.36 \pm 0.58$ & $\ldots$ & $\ldots$ & $\ldots$ & $\ldots$ & $\ldots$ \\
\hline 36.90 & $\ldots$ & $\ldots$ & $2.47 \pm 0.28$ & $\ldots$ & $\ldots$ & $\ldots$ & $\ldots$ \\
\hline 38.88 & $\ldots$ & $1.01 \pm 0.37$ & & $\ldots$ & $\ldots$ & $\ldots$ & $\ldots$ \\
\hline 38.89 & $\ldots$ & $\ldots$ & $1.83 \pm 0.31$ & $-0.93 \pm 6.74$ & $\ldots$ & $\ldots$ & $\ldots$ \\
\hline 38.90 & $-0.07 \pm 0.26$ & $\ldots$ & & $\ldots$ & $\ldots$ & $\ldots$ & $\ldots$ \\
\hline 39.90 & $\ldots$ & $0.80 \pm 0.27$ & $2.06 \pm 0.23$ & $\ldots$ & $-0.16 \pm 0.71$ & $\ldots$ & $-0.89 \pm 1.43$ \\
\hline 42.92 & $\ldots$ & $\ldots$ & $3.12 \pm 2.86$ & $-1.66 \pm 1.02$ & $\ldots$ & $\ldots$ & $\ldots$ \\
\hline 44.88 & $0.29 \pm 0.19$ & $\ldots$ & $1.64 \pm 0.27$ & $\ldots$ & $\ldots$ & $\ldots$ & $\ldots$ \\
\hline 46.89 & & $0.62 \pm 0.35$ & $1.47 \pm 0.41$ & $\ldots$ & $\cdots$ & $\ldots$ & $\ldots$ \\
\hline 50.87 & $0.22 \pm 0.34$ & $\ldots$ & $0.40 \pm 0.43$ & $\ldots$ & $\ldots$ & $\ldots$ & $\ldots$ \\
\hline 51.88 & $\ldots$ & $-0.20 \pm 0.34$ & $0.88 \pm 0.38$ & $\ldots$ & $1.95 \pm 1.12$ & $\ldots$ & $\ldots$ \\
\hline 52.90 & $\ldots$ & $\ldots$ & $1.34 \pm 0.55$ & $\ldots$ & $\ldots$ & $\ldots$ & $\ldots$ \\
\hline 53.88 & $\ldots$ & $1.59 \pm 0.44$ & $1.40 \pm 0.45$ & $\ldots$ & $0.69 \pm 1.10$ & $\ldots$ & $\ldots$ \\
\hline 54.88 & $0.94 \pm 0.56$ & $\ldots$ & $0.70 \pm 0.43$ & $\ldots$ & $2.76 \pm 1.74$ & $\ldots$ & $\ldots$ \\
\hline 56.88 & $\ldots$ & $-0.97 \pm 0.91$ & $1.91 \pm 0.53$ & $\ldots$ & $0.39 \pm 1.28$ & $\ldots$ & $\ldots$ \\
\hline 57.87 & $\cdots$ & $\ldots$ & $-0.04 \pm 0.63$ & $\cdots$ & $\ldots$ & $\ldots$ & $\cdots$ \\
\hline 58.87 & $\ldots$ & $0.32 \pm 0.47$ & $1.10 \pm 0.49$ & $\ldots$ & $1.26 \pm 1.13$ & $\ldots$ & $\ldots$ \\
\hline 59.87 & $-0.21 \pm 0.25$ & $\ldots$ & $1.20 \pm 0.42$ & $\ldots$ & $\ldots$ & $\ldots$ & $\ldots$ \\
\hline 65.86 & $0.38 \pm 0.34$ & $\ldots$ & $0.85 \pm 0.49$ & $\ldots$ & $\ldots$ & $\ldots$ & $\ldots$ \\
\hline 66.86 & $\ldots$ & $-0.66 \pm 0.59$ & $0.77 \pm 0.66$ & $\ldots$ & $\ldots$ & $\ldots$ & $\ldots$ \\
\hline 215.15 & $\ldots$ & $0.06 \pm 0.29$ & $0.11 \pm 0.33$ & $\ldots$ & $\ldots$ & $\ldots$ & $\ldots$ \\
\hline 217.15 & $-0.02 \pm 0.19$ & & $-0.04 \pm 0.28$ & $-0.87 \pm 0.87$ & $\ldots$ & $\ldots$ & $\ldots$ \\
\hline 219.14 & $\ldots$ & $-0.02 \pm 0.23$ & $0.47 \pm 0.26$ & $1.51 \pm 0.86$ & $\cdots$ & $\ldots$ & $\cdots$ \\
\hline 221.11 & $0.21 \pm 0.32$ & $\ldots$ & $0.16 \pm 0.59$ & $\ldots$ & $\ldots$ & $\ldots$ & $\ldots$ \\
\hline 223.14 & & $0.13 \pm 0.25$ & $0.67 \pm 0.30$ & $1.42 \pm 0.94$ & $\ldots$ & $\ldots$ & $\ldots$ \\
\hline 229.14 & $0.07 \pm 0.17$ & $\ldots$ & $0.53 \pm 0.25$ & $-0.50 \pm 0.80$ & $\ldots$ & $\ldots$ & $\ldots$ \\
\hline 231.13 & $\ldots$ & $0.92 \pm 0.31$ & $0.51 \pm 0.41$ & $\ldots$ & $\ldots$ & $\ldots$ & $\ldots$ \\
\hline 303.53 & $\cdots$ & & $\ldots$ & $-0.64 \pm 0.39$ & $1.17 \pm 0.56$ & $1.96 \pm 0.70$ & $1.49 \pm 1.20$ \\
\hline 306.52 & $\cdots$ & $0.18 \pm 0.15$ & $\ldots$ & $\ldots$ & $\ldots$ & $\ldots$ & $\ldots$ \\
\hline 314.00 & $\ldots$ & $\ldots$ & $-0.09 \pm 0.09$ & $\ldots$ & $\ldots$ & $\ldots$ & $\ldots$ \\
\hline 353.44 & $0.04 \pm 0.09$ & $\ldots$ & $\ldots$ & $\ldots$ & $\ldots$ & $\ldots$ & $\ldots$ \\
\hline 1152.86 & $\ldots$ & $-0.01 \pm 0.00$ & $\ldots$ & $\ldots$ & $\ldots$ & $\ldots$ & $\ldots$ \\
\hline 1153.36 & $\ldots$ & $\ldots$ & $-0.02 \pm 0.19$ & $0.03 \pm 0.54$ & $-0.02 \pm 0.72$ & $0.09 \pm 1.12$ & $5.53 \pm 7.37$ \\
\hline 1153.85 & $-0.01 \pm 0.00$ & $\cdots$ & & & 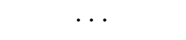 & (2008 & (1) \\
\hline
\end{tabular}

